\title{
Numerical Simulations of Shoaling Internal Solitary Waves of Elevation
}

by

\author{
Chengzhu Xu
}

\author{
A thesis \\ presented to the University of Waterloo \\ in fulfillment of the \\ thesis requirement for the degree of \\ Master of Mathematics \\ in \\ Applied Mathematics
}

Waterloo, Ontario, Canada, 2015

(c) Chengzhu Xu 2015 


\section{Author's Declaration}

I hereby declare that I am the sole author of this thesis. This is a true copy of the thesis, including any required final revisions, as accepted by my examiners.

I understand that my thesis may be made electronically available to the public. 


\begin{abstract}
We present high-resolution, two- and three-dimensional direct numerical simulations of laboratory-scale, fully nonlinear internal solitary waves of elevation shoaling onto and over a small-amplitude shelf. The three-dimensional, mapped coordinate, spectral collocation method used for the simulations allows for accurate modelling of both the shoaling waves and the bottom boundary layer. We focus on wave-induced instabilities during the shoaling and de-shoaling processes. The shoaling of the waves is characterized by the formation of a quasi-trapped core which undergoes a spatially growing stratified shear instability at its edge and a lobe-cleft instability in its nose. Both of these instabilities develop and three-dimensionalize concurrently, leading to strong bottom shear stress. During the deshoaling process, the core breaks up and ejects fluid that forms a vortex-rich region near the down-sloping portion of the shelf. The flow in this region is highly turbulent and the bottom shear stress is extremely strong. Experiments with a corrugated bottom boundary are also performed. Boundary layer separation is found inside each of the corrugations during the wave's shoaling process. Our analyses suggest that all of these wave-induced instabilities can lead to enhanced turbulence in the water column and increased shear stress on the bottom boundary. Through the generation and evolution of these instabilities, the shoaling and de-shoaling cycles of internal solitary waves of elevation are likely to provide systematic mechanisms for material mixing and sediment resuspension. These mechanisms have significant environmental implications on the near-coastal regions of the world's oceans.
\end{abstract}




\section{Acknowledgements}

There are many people to whom I owe thanks during my graduate studies, without their support this thesis would not be possible. First and foremost, I would like to thank my supervisor Dr. Marek Stastna who has guided me to grow as a researcher, encouraged me to look ahead to my academic career with confidence, and provided me with valuable help and great patience when I made silly mistakes.

I would also like to thank my committee members Dr. Hans De Sterck and Dr. Francis Poulin for taking their time to review my thesis and provide valuable suggestions. I would like to thank Dr. Dmitry Pelinovsky for his inspiration that led me to the world of numerical analysis and fluid mechanics. I would like to thank Chris Subich for his comments and suggestions on the numerical simulations I performed for this thesis.

Many thanks to everyone who is and was in our office and the fluids lab, especially to David for the badminton sessions on Saturday mornings and the first Hockey game I have ever watched, to Ben for proof reading my writings and answering my latex and python questions, to Kevin for the potluck in Christmas, to Yangxin for running my tutorial in this coldest February while I was in California, to Jared for buying me beer in the AGU conference and taking care of my poster when I left for China, to Nancy for teaching me how to run SPINS on SciNet, and to Wentao for teaching me the most basic Linux commands. Thanks also go to the administration and staff in the Department, especially to Maureen for the Turkey dinner on the Thanksgiving day.

Most importantly, I would like to thank my family and friends. I thank my parents who have always been trying their best to provide me with high quality education, and understand and encourage me no matter what career path I choose. I thank my uncle Otto for his advices on my life and study in Canada through numerous international long distance phone calls. I also thank all of my friends, especially those on the other side of the Pacific Ocean who have proven that friendship never fades away regardless of the distance and time that sets us apart. I thank my wife Ellie most of all, for everything we have been going through together and every effort you have made for our better future, not to mention that you travelled half the world to be part of my life and pursue our dream together. It is you who brought all the colors to my life. 


\section{Dedication}

To my wife, Ellie. 


\section{Table of Contents}

List of Tables $\quad$ viii

List of Figures $\quad$ ix

1 Introduction $\quad 1$

1.0.1 Plan of Thesis . . . . . . . . . . . . . . . . 4

2 Theoretical Background and Computational Approach 5

2.1 Equations of Fluid Motion . . . . . . . . . . . . . . . . 7

2.2 Dubreil-Jacotin-Long Equation . . . . . . . . . . . . . . . 10

2.3 Numerical Method and Model Description . . . . . . . . . . . . . . 14

2.4 Shear Instability . . . . . . . . . . . . . . . . . . . 15

2.4.1 Numerical solutions . . . . . . . . . . . . . . 17

2.5 Bottom Shear Stress . . . . . . . . . . . . . . . . . 20

3 Model Setup and Two-Dimensional Simulations 22

3.1 Model Setup and Simulation Parameters . . . . . . . . . . . . . . 23

3.2 The Base Case . . . . . . . . . . . . . . . . . . . 25

3.2 .1 Shear Instability . . . . . . . . . . . . . . 26

3.2.2 Bottom Shear Stress . . . . . . . . . . . . . . . 30

3.3 Medium and Higher Pycnoclines . . . . . . . . . . . . . . . . . . 32 
3.3.1 Shoaling Behavior . . . . . . . . . . . . . . . . . 33

3.3.2 Wave Breaking and the Vortex-Rich Region . . . . . . . . . . 35

3.3.3 The Higher Pycnocline Case . . . . . . . . . . . . . . . . . . . . 39

3.4 A Corrugated Bottom Boundary . . . . . . . . . . . . . . . . . 41

4 Three-Dimensional Simulations 44

4.1 The Base Case . . . . . . . . . . . . . . . . . . . . . 48

4.1.1 Three-Dimensional Flow Structure . . . . . . . . . . . . 48

4.1.2 Shoaling Behavior and Boundary Layer Interaction . . . . . . . . . 53

4.2 The Medium Pycnocline Case . . . . . . . . . . . . . . . . . . . 58

4.2 .1 Lobe-Cleft Instability . . . . . . . . . . . . . . . . 58

4.2.2 The Vortex-Rich Region . . . . . . . . . . . . . . . . 60

$\begin{array}{lll}5 & \text { Conclusions } & 67\end{array}$

$\begin{array}{ll}\text { References } & 72\end{array}$ 


\section{List of Tables}

3.1 List of parameters for $2 \mathrm{D}$ simulations, where $\eta_{\max }$ is the maximum isopycnal displacement which measures the wave amplitude, and $c$ is the ISW propagation speed. The minimum values of $w$ is equal to $-w_{\max }$ by symmetry. All wave parameters are obtained from solutions of the DJL equation and are for the initial waves only (i.e. at $t=0 \mathrm{~s}$ ). Parameters fixed for all simulations are not shown here. . . . . . . . . . . . . . . . .

4.1 List of numerical parameters and summary of simulation results of 3D simulations. Cases 1-4 are initialized from the 2D Base Case, and Medium is initialized from the 2D Medium pycnocline case. In summary, in the Base Case the Gibbs phenomenon is observed in the cases with larger diffusivity, the generation of lobe-cleft instability occurs before $t=41 \mathrm{~s}$ so that Case 1 is physically irrelevant, and the three-dimensionalizaiton of the KelvinHelmholtz billows is absent with small domain width. . . . . . . . . . . 


\section{List of Figures}

2.1 Schematic diagram of internal solitary wave propagation. . . . . . . . . 11

2.2 Background profiles of $(a)$ the horizontal velocity and $(b)$ the density for a two dimensional inviscid stratified parallel shear flow. The resulting Richardson number is shown in $(c)$ with the dashed line indicating the critical Richardson number $R i=0.25$. . . . . . . . . . . . . . . . 16

2.3 Perturbation profiles of the shear flow. Panel $(a)$ : growth rate of waves of disturbance versus wave length. Panel $(b)$ : real part of the stream function corresponding to the fastest growing wave, determined by equation (2.46).

3.1 Schematic diagram showing the computational domain and model setup. The origin is fixed at the upper left corner of the domain, and the unit for both axes is meter. The bottom topography is given by equation (3.1) with the crest located at $(x, z)=(6,-0.175) \mathrm{m}$. The pycnocline is centered at $z=z_{0} \mathrm{~m}$ and has a thickness $d=0.02 \mathrm{~m}$. The initial wave propagates to

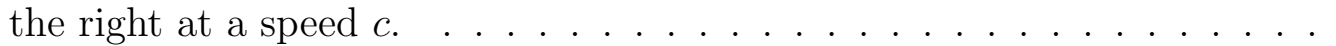

3.2 Normalized density contours showing $(a)$ the initial wave and ( $b$ ) its propagation and shoaling. Here and henceforth, the color axes of all density plots are saturated at $\rho=1 \pm 0.01$, with dense fluids shown in red, light fluid shown in blue and midrange values shown in green. . . . . . . . . . .

3.3 Three panels of shaded density contours showing the propagation and shoaling of the wave and the development of the core at $(a) t=39 \mathrm{~s},(b) t=40$ $\mathrm{s}$ and $(c) t=41 \mathrm{~s}$. The dashed line in panel $(a)$ is located at $x=5.29 \mathrm{~m}$, along which the analysis of shear instability is given in figure 3.5. Details of the billow and the wave front as indicated by the boxes in panel $(c)$ are

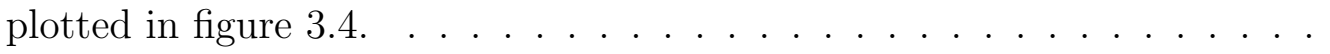


3.4 Detailed density contours of $(a)$ one of the billows at the edge of the core and $(b)$ the wave front and the bottom boundary layer at $t=41 \mathrm{~s}$. See

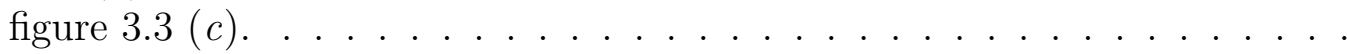

3.5 Analysis of shear instability along $x=5.29 \mathrm{~m}$ at $t=39 \mathrm{~s}$ (the dashed line in figure $3.3(a))$. Panel $(a)$ : background horizontal velocity profile. Panel $(b)$ : squared buoyancy frequency, where the negative value at $z \approx-0.175$ $\mathrm{m}$ is due to the no-slip boundary condition (see figure 3.4 $(b)$ ). Panel $(c)$ : growth rate of waves of disturbance. Panel $(d)$ : filled contour plot of the stream function showing the $2 \mathrm{D}$ structure of the fastest growing wave. . . .

3.6 Analysis of shear instability along $x=5.38 \mathrm{~m}$ at $t=39 \mathrm{~s}$. Panel $(a)$ : growth rate of waves of disturbance. Panel $(b)$ : filled contour plot of the stream function showing the $2 \mathrm{D}$ structure of the fastest growing wave. . . . . . . .

3.7 Evolution of the bottom shear stress during the wave passage. All subplots are normalized by the maximum shear stress found at $t=35 \mathrm{~s}(a)$. The average slope of the bottom boundary between $x=4.4 \mathrm{~m}$ and $6 \mathrm{~m}$ is

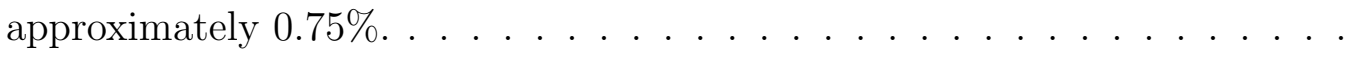

3.8 Shaded density contours of the simulation labeled as Medium showing the wave propagation at $(a) t=50 \mathrm{~s},(b) t=60 \mathrm{~s}$ and $(c) t=70 \mathrm{~s}$. Note that periodic boundary conditions are used in the $x$-direction with a period $L_{x}=7 \mathrm{~m} \ldots \ldots \ldots \ldots \ldots \ldots$

3.9 Vertical velocity plot at $t=50 \mathrm{~s}$. The color axis is saturated at $w= \pm 0.3$ $\mathrm{cm} / \mathrm{s}$, with positive (upward) velocity shown in red and negative (downward) velocity shown in blue. Note that the maximum and minimum vertical velocities at $t=50 \mathrm{~s}$ are $w_{\max }=3.84 \mathrm{~cm} / \mathrm{s}$ and $w_{\min }=-3.37 \mathrm{~cm} / \mathrm{s}$, respectively. 34

3.10 Density contour plots of the wave at $t=50 \mathrm{~s}$ obtained from $(a)$ the simulation result and $(b)$ the solution of the DJL equation with the bottom boundary located at $z=-0.175 \mathrm{~m}$, the crest of the bottom topography in the simulation domain. . . . . . . . . . . . . . . . .

3.11 Comparison of the density profiles of $(a)$ the newly generated wave at $t=80$ $\mathrm{s}$ and $(b)$ the initial wave at $t=0 \mathrm{~s}$. Because of the periodic boundary condition, these waves are essentially at the same location. . . . . . . . . 
3.12 Vorticity field plots from $t=54 \mathrm{~s}$ to $62 \mathrm{~s}$. The color axis is saturated at $\omega= \pm 15 \mathrm{~s}^{-1}$, with positive vorticity shown in red (hot), negative vorticity shown in blue (cold) and irrotational flow shown in black. The pycnocline is not shown here because the plots will otherwise be too busy. Nevertheless, location of the wave crest can be visualized by the negative baroclinic vorticity at $z \approx-0.1 \mathrm{~m}$. Detail of the vortex-rich region as indicated by the white box in panel $(d)$ is given in figure $3.13 \ldots \ldots \ldots \ldots \ldots$

3.13 Detail of the vortex-rich region and the bottom boundary layer at $t=60$ s given by $(a)$ vorticity field, $(b)$ horizontal velocity and $(c)$ bottom shear stress plots. For panel $(a)$ the color axis is the same as figure 3.12, whereas for panel $(b)$ it is saturated at $\pm 0.1 \mathrm{~m} / \mathrm{s}$ with positive velocity shown in red, negative velocity shown in blue and zero velocity shown in green. Panel $(c)$ is normalized by the maximum positive bottom shear stress found in the plot. 38

3.14 Density contours showing the de-shoaling of the wave and the formation of the wave train in the Higher pycnocline case at $(a) t=50 \mathrm{~s}$, (b) $t=70 \mathrm{~s}$

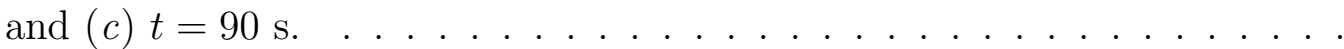

3.15 Vorticity field of the Rough bottom boundary case at $t=50 \mathrm{~s}$. The the color axis is the same as in figure 3.12. The solid curve denotes the location of the pycnocline. Detail of the flow in the boundary layer is given in figure 3.16 .

3.16 Detail of the bottom boundary layer at $t=50 \mathrm{~s}$ given by $(a)$ vorticity field, (b) horizontal velocity and $(c)$ bottom shear stress plots. For panel $(a)$ the color axis is the same as figure 3.12, whereas for panel $(b)$ it is saturated at $\pm 0.04 \mathrm{~m} / \mathrm{s}$ with positive velocity shown in red, negative velocity shown in blue and zero velocity shown in green. Panel $(c)$ is normalized by the maximum positive bottom shear stress found in the plot. . . . . . . . . .

4.1 Density isosurface corresponding to $\rho=1$ showing the 3D structure of the shoaling wave at $t=42 \mathrm{~s}$. The vertical slice in the background shows the shaded density profile in the $x-z$ plane at $y=0.1 \mathrm{~m} . \ldots \ldots \ldots \ldots$

4.2 Spanwise average of the density field at $t=44 \mathrm{~s}$. Cross section views of the density profile corresponding to the dashed lines are given in figure 4.3 . . . 
4.3 Cross section views of the density field at $t=44 \mathrm{~s}$, with the $y$ - $z$ plane views showing $(a)$ the billows, $(b)$ the core's head and $(c)$ nose, and the $x$-y plane views showing $(d)$ the high shear region at the edge of the wave and $(e)$ the bottom boundary layer. The dashed lines indicate the corresponding locations of the planes in different views. . . . . . . . . . . . . . .

4.4 Vertical velocity profile at $t=44 \mathrm{~s}$. Panel $(a)$ : spanwise average. Panels (b)-(e): $x-y$ plane views corresponding to the horizontal layers indicated by the dashed lines in panel $(a)$. Note that due to the topography of the bottom boundary and the mapping used in the spectral method, these layers are not exactly parallel to the $x-y$ plane in the physical domain. Here, the bottom boundary is located at $z \approx-0.175 \mathrm{~m}$ with an average slope of $0.46 \%$. The color axis is saturated at $\pm 8 \mathrm{~cm} / \mathrm{s}$ with downward velocity shown in blue and upward velocity shown in red. . . . . . . . . . . . . . . . . . .

4.5 Normalized standard deviation of the kinetic energy field in the spanwise direction at $(a) t=40 \mathrm{~s},(b) t=42 \mathrm{~s}$ and $(c) t=44 \mathrm{~s}$. The dark regions represent a value of zero, while the highlights are saturated at $50 \%$ of the maximum value found in these plots. . . . . . . . . . . . . . . . . .

4.6 Normalized bottom shear stress at $(a) t=40 \mathrm{~s},(b) t=42 \mathrm{~s}$ and $(c) t=44$ s. Solid lines represent results obtained from the 3D simulation, and dashed lines represent results obtained from the 2D simulation. Spanwise averages are used in the $3 \mathrm{D}$ case. . . . . . . . . . . . . . . . . . . . . .

4.7 Normalized shear stress histories on the bottom boundary in $(a)-(b)$ the streamwise direction and $(c)-(d)$ the spanwise direction. For all subplots, the color axis is saturated at $\pm 60 \%$ of the maximum along-hill shear stress observed in panel $(b)$, with positive values shown in red (hot), negative values shown in blue (cold) and intermediate values shown in black. . . . .

4.8 Spanwise average of the density field at $t=50 \mathrm{~s}$. Cross section views of the density profile corresponding to the dashed lines are given in figure 4.9. . .

4.9 Cross section views of the density field at $t=50 \mathrm{~s}$ corresponding to the locations indicated by the dashed lines in figure 4.8, with $(a)-(c)$ the $y-z$ plane views showing the lobe-cleft instability, and $(d)$ the $x-y$ plane view showing the boundary layer located at $3 \mathrm{~mm}$ above the bottom boundary. Note that the bottom boundary layer is not exactly parallel to the $x$ - $y$ plane due to the topography. . . . . . . . . . . . . . . . . . . . 
4.10 Spanwise average of the normalized enstrophy plots showing the wave's deshoaling process. The brightness in these plots is proportional to the magnitude of the enstrophy field. The pycnocline is indicated by the solid curves. 61

4.11 Spanwise average of the normalized difference between the enstrophy and the $y$-component of the vorticity (i.e. the quantity $Q_{y}$ defined in equation (4.6)) corresponding to figure $4.10 . \ldots \ldots \ldots \ldots \ldots$

4.12 Normalized $z$-vorticity plots along the slice $2 \mathrm{~mm}$ above the bottom boundary showing the evolution of the BBL during the de-shoaling of the wave. The positive (negative) vorticity is shown in red (blue) with the magnitude indicated by the brightness. The $x$-vorticity at the slices indicated by the dashed lines is given in figure $4.14 \ldots \ldots \ldots \ldots \ldots$

4.13 Normalized along-topography bottom shear stress. The positive (negative) stress is shown in red (blue) with the magnitude indicated by the brightness. 64

4.14 Normalized $x$-component of the vorticity field in the $y$ - $z$ plane at $(a) t=50$ $\mathrm{s}, x=6.4 \mathrm{~m},(b) t=54 \mathrm{~s}, x=6.4 \mathrm{~m},(c) t=54 \mathrm{~s}, x=6.6 \mathrm{~m}$ and $(d) t=58$ $\mathrm{s}, x=6.6 \mathrm{~m}$, corresponding to the dashed lines in figure 4.12 . The color axis is the same as figure $4.12 \ldots \ldots \ldots \ldots \ldots$

5.1 Vertical velocity plots for the Scale-up case at $t=40 \mathrm{~s}$. Panel $(a)$ : overall flow structure. Panel $(b)$ : surface boundary layer behind the main wave. Panel $c$ ): bottom boundary layer underneath the wave. . . . . . . . . 


\section{Chapter 1}

\section{Introduction}

Internal waves are waves that exist in the interior, rather than on the surface, of a stratified fluid. A stratified fluid usually contains several layers of fluid of different physical properties. For example, through temperature and salinity variation, the Earth's ocean is a density stratified medium. Indeed, density stratification is the most commonly considered type of stratification, which can be either discrete (such as a layer of oil on water) or continuous. For a continuously stratified fluid, the density profile may contain one or more regions of rapid change, called pycnoclines, that separate the light fluid in the upper layer from the heavy fluid in the lower layer. Waves propagating horizontally along the pycnocline are called vertically trapped internal waves. The structure of internal waves is determined by the location of the pycnocline: waves of depression are associated with a pycnocline that is centered above the mid-depth of the water column, whereas waves of elevation occur when the pycnocline is centered below the mid-depth. Because the density difference within the same fluid is usually much smaller than that across the interface of two fluids (e.g. air and water), the restoring forces are also weaker. Hence, for the same amount of energy, internal waves may attain an amplitude much larger than surface wave amplitudes, and in some cases form a recirculating, or trapped, core.

Internal solitary waves (ISWs) are commonly observed in the Earth's atmosphere and oceans. These waves may propagate over a long distance without changing shape (i.e. they are solitary-like). ISWs with trapped cores can provide a very effective mechanism for the transport of fluid and materials, and hence have significant environmental impact on coastal regions of the world's oceans. Large-amplitude solitary-like waves of elevation propagating along the ocean bottom were observed on Oregon's continental shelf (Klymak and Moum, 2003) and in Massachusetts Bay (Scotti and Pineda, 2004). Trapped cores transporting parcels of fluid and sediments were found in both observations. Since 
waves of elevation are formed close to the ocean bottom, they may contain trapped cores interacting directly with the bottom boundary layer (BBL). They are thus expected to provide potential for significant material mixing across the BBL and in extreme cases, sediment resuspension. ISW-induced mixing and resuspension was observed on the California Shelf (Bogucki et al., 1997), where the wave passage was accompanied by an increased concentration of particulates in the water column. Since the near-bottom current speed was much lower than the traditionally expected threshold value for sediment resuspension, this observation suggests that the ISW and ISW-induced unsteady flow can potentially be responsible for both mixing and resuspension.

Lamb $(2002,2003)$ showed that the formation of ISWs with trapped cores is closely connected to the limiting behaviour of these waves. According to the fully nonlinear theory (details will be discussed in section 2.2), the maximum displacement of the pycnocline (i.e. the maximum amplitude) of the wave cannot exceed the mid-depth of the water column. Hence, large-amplitude waves are usually broad, having a nearly horizontal flow in their center. Such a flow is called a conjugate flow, and the limiting behavior of the wave in this case is called the conjugate-flow limit. However, if the maximum horizontal current in the wave exceeds the wave propagation speed, then the wave is said to reach the breaking limit and a trapped core will be formed. A large-amplitude solitary wave can reach the breaking limit if there is significant stratification at the relatively thinner layer (i.e. the bottom layer in the case of waves of elevation or the surface layer in the case of waves of depression). In contrast, waves are usually conjugate-flow limited when the surface (or bottom) layer is well mixed.

In addition to the stratification, other properties of the flow field such as the presence of a background current may also affect the limiting behavior of internal waves. Stastna and Lamb (2002) found that, for a given stratification, the presence of a shear background current does not only change the wave's width and propagation speed, but also affects the nature of the upper bound on wave amplitude. In other words, waves that may be broadening limited without a background current could be breaking limited with a background current and vice versa.

While the theoretical description of trapped cores suggests that they are fairly quiescent (Derzho and Grimshaw, 1997), during their formation, these cores may generate hydrodynamic instabilities. Two-dimensional numerical simulations suggest that shear instability (Helfrich and White, 2010; Carr et al., 2012) and boundary layer instability (Diamessis and Redekopp, 2005; Stastna and Lamb, 2008) can occur in both waves of elevation and depression. The shear instability occurs along the edge of the cores and takes the form of Kelvin-Helmholtz billows, leading to fluid exchange between the cores and the ambient flow. The boundary layer instability can cause elevated levels of bottom shear stress and 
a periodic shedding of coherent vortex structures, thereby contributing to the sediment resuspension and mixing in the water column. We should point out though, it is unclear to what extent these instabilities may develop in three dimensions. In fact, depending on the model setup, three-dimensional flows may behave significantly differently from their two-dimensional counterparts.

The shoaling of internal waves over bottom topography is one of the most important mechanisms responsible for the generation of wave-induced instabilities. In the laboratory experiments performed by Boegman et al. (2005), high-frequency internal wave breaking was observed along sloping topography, which led to energy transfer from the wind-forced basin-scale motions to the turbulent motions. Aghsaee et al. (2012) showed, using twodimensional numerical simulations, that for waves of depression, boundary layer instability in the form of separation bubble bursting that leads to vortex shedding can be generated during shoaling. The instability can eventually reach the pycnocline, modifying the wave breaking mechanism, though it is unclear to what extent three-dimensional effects will modify this observation. For periodically forced internal waves, three-dimensional simulations performed by Venayagamoorthy and Fringer (2007) suggest that interaction of shoaling waves with the bottom topography results in the formation of upslope-surging vortex cores of dense fluid, the so-called internal boluses, that propagate as gravity currents onto the shelf. Behaviour typical of gravity currents, such as the lobe-cleft instability, is observed in these boluses. The generation of lobe-cleft instability is driven by the propagation of the gravity current head (or the bolus) together with the presence of a density gradient in the BBL. A detailed investigation on the lobe-cleft instability is given in Hartel et al. (2000a,b), and these papers will be discussed in the relevant sections of chapter 4 .

Even if the presence of bottom topography does not lead to a shoaling wave, it can still alter the behavior of the wave and wave-induced instabilities. The interaction of ISWs with a step topography were studied in Maderich et al. (2010) and Talipova et al. (2013) using two-dimensional simulations. Waves of depression were considered in the first paper, which suggests that the wave-step interaction leads to strong eddy generation such that the fluid from below the step is mixed into the layer overlying the step. In the second paper where the results are extended to both waves of depression and elevation, quantitative measurement of energy loss of due to wave-bottom topography interaction is calculated for different regimes. For smooth topography with much more gentle slopes, Harnanan et al. (2015) performed three-dimensional simulations and showed that there exist two qualitatively different modes of instability. The first is a separation instability, which leads to strong, localized vortex roll-up. This instability has a qualitative similarity to that in Maderich et al. (2010) and Talipova et al. (2013), though is far less violent. The second type of instability over broad topography that is far more typical of the field is a vortex 
roll up instability. The ISW-induced BBL jet, which occurs behind the wave body, rolls up after the main wave body passes over the hill, leading to enhanced bottom shear stress, systematic pumping of fluid out of the BBL, and secondary vorticity production.

\subsubsection{Plan of Thesis}

This thesis will contribute to the study of ISWs propagating over bottom topography. Fully nonlinear mode-1 waves of elevation are simulated using high-resolution two- and threedimensional simulations based on a spectral collocation method. Both the shoaling and de-shoaling processes of the waves are studied. As the wave shoals, it forms a quasi-trapped core, which undergoes a spatially growing stratified shear instability at its edge and a lobecleft instability in its nose. Both of these instabilities develop and three-dimensionalize concurrently and enhance the bottom shear stress. During the de-shoaling process, the core breaks up and the fluid ejected from the core forms a vortex-rich region near the downsloping portion of the shelf, in which the flow is highly turbulent and the bottom shear stress is extremely strong. The influence of a rough bottom boundary on the behaviour of shoaling waves is also studied.

The remainder of the thesis is organized as following. Chapter 2 outlines the theory of internal waves and briefly describes the numerical model used in this study. The model setup and two-dimensional simulations with different background stratifications is discussed in chapter 3. Three-dimensional simulations of two particular cases are performed and discussed in chapter 4 . Chapter 5 summarizes findings of this study and proposes future research directions. 


\section{Chapter 2}

\section{Theoretical Background and Computational Approach}

In the study of fluid dynamics, three approaches have been most commonly used: measurement (including field observations and laboratory experiments), theoretical analysis and numerical simulation. In this chapter we shall focus on the theoretical and numerical approaches and provide some fundamental tools for analyses to be discussed in this thesis.

The equations governing fluid flows are highly nonlinear, and thus have no analytical solutions in general. For this reason, theoretical analysis is usually classified as one of the three categories: linear theory, fully nonlinear theory and weakly nonlinear theory. In the linear theory, all nonlinear terms in the governing equations are eliminated in order to make the equations solvable. In other words, the nonlinear effect in the fluid flow is assumed to be negligible. Since this assumption is rarely true in the real world, in most cases results predicted by the linear theory do not agree with measurements. Nevertheless, it does provide some useful insight into the properties of fluid flows. For example, consistent concepts such as phase and group velocity (Kundu et al., 2012, section 7.5) as well as other mathematical machinery are developed from the linear theory.

On the contrary, fully nonlinear theory does not make any assumptions with respect to nonlinearity, and hence the governing equations can only be solved numerically. When performed correctly, numerical simulations can provide fairly accurate results. There are limitations as well, primarily due to the available computational resources. Until relatively recently, well-resolved simulations of nonlinear flows are restricted to two dimensions, especially at scales relevant for environmental flows. Even with today's computational power, three-dimensional simulations are still time consuming, and the output data may require 
gigabytes or even terabytes of storage space. In fact, the nonlinearity is one of the key factors that makes simulations computationally expensive, because the range of length scales presented in the fully nonlinear theory is often very large.

For flows in which nonlinear effects only play a secondary role, weakly nonlinear theory is the alternative to fully nonlinear theory. For uni-directional wave propagation with relatively small amplitude, the Korteweg-de Vries (KdV) equation (Korteweg and de Vries, 1895) is probably the most widely used weakly nonlinear model. Derived using the asymptotic theory, the KdV equation is a nonlinear partial differential equation (PDE) that can be solved exactly. As such, the mathematical significance of the KdV theory perhaps outweighs its importance in the application of fluid dynamics. However, similar to any other weakly nonlinear models, we can only expect the KdV equation to perform well within a restricted set of problems. The solutions of the KdV equation for large amplitude waves have been shown to be different from wave forms predicted by the full set of governing equations. For details of the KdV theory and a comparison with the fully nonlinear theory, see e.g. Lamb and Yan (1996); Lamb (1999).

In this chapter, we will derive internal wave theory based on the fully nonlinear theory, and briefly outline the numerical model used in this work: Spectral and Pseudo-spectral Incompressible Navier-Stokes Solver (SPINS). The theory of shear instability and the method for computing the bottom shear stress will also be introduced in this chapter.

In this work, the numerical simulations are performed on the laboratory scale. The fluid (i.e. water) is assumed to be incompressible and the effect of the Earth's rotation is neglected. Also, we will only consider the Cartesian coordinate system, such that the position and velocity vectors can be expressed as $\boldsymbol{x}=(x, y, z)$ and $\boldsymbol{u}=(u, v, w)$, respectively. More specifically, the $x, y$ and $z$ axes are directed along the streamwise, spanwise and vertical directions, respectively. Along the $x$-axis, the upstream direction is defined as the direction toward which the wave propagates (i.e. ahead of the wave, which is positive infinity in our simulations), and the downstream direction is defined as the reverse direction. The $x$-direction is also called the along-topography direction, since we will consider bottom topography in the $x$-direction only. Unless otherwise specified, the surface of the fluid is always fixed at $z=0$. 


\subsection{Equations of Fluid Motion}

Under the Boussinesq approximation, the incompressible Navier-Stokes equations are (Kundu et al., 2012)

$$
\begin{aligned}
\frac{D \boldsymbol{u}}{D t} & =-\nabla p+\rho \boldsymbol{g}+\nu \nabla^{2} \boldsymbol{u}, \\
\nabla \cdot \boldsymbol{u} & =0, \\
\frac{D \rho}{D t} & =\kappa \nabla^{2} \rho,
\end{aligned}
$$

where $D / D t$ is the material derivative defined by

$$
\frac{D}{D t}=\frac{\partial}{\partial t}+\boldsymbol{u} \cdot \nabla
$$

Equation (2.1a) is the momentum conservation equation, where $\boldsymbol{u}$ is the fluid velocity, $\rho$ is the density, $p$ is the pressure, $\boldsymbol{g}=(0,0,-g)$ with $g \approx 9.81 \mathrm{~m}^{2} / \mathrm{s}$ is the gravitational acceleration and $\nu$ is the kinematic viscosity. Note that in this equation both $\rho$ and $p$ are scaled by some reference density $\rho_{0}$, and the dynamic viscosity $\mu$ is defined by $\mu=\rho_{0} \nu$. Equation (2.1b) is the continuity equation which describes the conservation of mass for an incompressible fluid. Equation (2.1c) describes the conservation of energy, where $\kappa$ is the mass diffusivity.

The Boussinesq approximation (Kundu et al., 2012, section 4.9) states that the density variation of an incompressible fluid can be neglected except in the gravity term, provided that the density different in the fluid is small and the vertical length scale is relatively small. It is applicable in environmental and geophysical fluid flows of water where the horizontal length scale is usually much larger than the vertical length scale. The Boussinesq approximation will be used throughout this thesis.

An important concept derived from the governing equations is vorticity dynamics. Vorticity is a vector that measures the circular motion (i.e. rotation) of fluid particle. The vorticity field of the flow, denoted by $\boldsymbol{\omega}$, is defined by the curl of the velocity:

$$
\boldsymbol{\omega}=\nabla \times \boldsymbol{u} .
$$

Similarly, the vorticity equation is obtained from the curl of the momentum equation:

$$
\frac{D \boldsymbol{\omega}}{D t}=(\boldsymbol{\omega} \cdot \nabla) \boldsymbol{u}+\nu \nabla^{2} \boldsymbol{\omega}+\nabla \times \rho \boldsymbol{g} .
$$


In this equation, the term $(\boldsymbol{\omega} \cdot \nabla) \boldsymbol{u}$ represents the stretching and tilting of vortex lines, $\nu \nabla^{2} \boldsymbol{\omega}$ represents the vorticity production due to viscosity, and $\nabla \times \rho \boldsymbol{g}$ is the baroclinic vorticity. This equation states that the rate of change of vorticity $D \boldsymbol{\omega} / D t$ is determined by the combined effect of these quantities.

We shall also introduce the buoyancy frequency $N(z)$, the frequency at which a vertically displaced parcel will oscillate within a stratified fluid when it is in hydrostatic equilibrium. The square of the buoyancy frequency is defined by

$$
N^{2}(z)=-\frac{d \bar{\rho}}{d z} g
$$

where $\bar{\rho}$ is the background or unperturbed density distribution and is a function of $z$ only. Note that for simplicity and consistency, $\bar{\rho}$ is also nondimensionalized by the reference density $\rho_{0}$, so that the term $1 / \rho_{0}$ in the standard definition of $N^{2}$ does not appear here. The buoyancy frequency measures the strength of the stratification: a strong stratification yields a rapid change in the density field and hence a large value of $N^{2}$, whereas a weak stratification leads to a small variation in $\bar{\rho}$ and hence a small $N^{2}$. Note that in a stably stratified fluid, $d \bar{\rho} / d z<0$ for all $z$ so that $N^{2}$ is always positive. If $d \bar{\rho} / d z>0$, then the stratification is unstable and instabilities may occur.

In two-dimensional flows, additional features are present such that many theoretical analyses can be further simplified. For example, the two-dimensional continuity equation can be written as

$$
u_{x}+w_{z}=0
$$

which is identically satisfied with the introduction of a stream function $\psi(x, z)$ such that $(u, w)=\left(\psi_{z},-\psi_{x}\right)$. Hence, the velocity field can be represented by a single variable and the problem is thus simplified. Here and henceforth, we use subscripts to denote partial derivatives in scalar equations, unless otherwise specified. As another example, consider the vorticity field of a two-dimensional flow, in which the only non-zero component is

$$
\omega=u_{z}-w_{x}=\nabla^{2} \psi
$$

The vorticity equation (2.4) also reduces to a single scalar equation:

$$
\omega_{t}+u \omega_{x}+w \omega_{z}=\nu\left(\omega_{x x}+\omega_{z z}\right)+\rho_{x} g .
$$

With these properties, theoretical analyses of fluid flows are much easier to carry out in two dimensions. In the remainder of this chapter, the theories of internal wave and shear instability will be presented in two dimensional domains. 
While the above equations are in dimensional forms, physical properties of fluid flows are independent of the units imposed on the variables. In the study of fluid dynamics, dimensional analysis provides an important technique for problem simplification and scaling analysis. The Reynolds number, defined by

$$
R e=\frac{U L}{\nu}
$$

where $U$ and $L$ are the characteristic velocity and length scales, is the most commonly used dimensionless number. It measures the ratio of the inertial force to the viscous force. A small Reynolds number means that the inertial force is relatively small and the the viscous force is important in determining the flow properties, whereas a large Reynolds number suggests that the viscous effect is negligible compared with the inertial force. Therefore, small Reynolds numbers are often associated with viscous flows, while large Reynolds numbers are typically found in turbulent flows. The choice of $L$ depends on the context of the problem. For example, the length scale of the flow away from rigid boundaries is usually much larger than that within boundary layers, and hence for flows away from boundary layers the viscous effect can be neglected. With this assumption, the viscous term can be dropped and equation (2.1a) simplifies to the Euler equation under the Boussinesq approximation:

$$
\frac{D \boldsymbol{u}}{D t}=-\nabla p+\rho \boldsymbol{g}
$$

Dimensionless numbers other than $R e$ are also important tools to characterize different flow properties. They will be defined and analyzed where necessary in the thesis. 


\subsection{Dubreil-Jacotin-Long Equation}

Though the equations governing fluid flows are not directly solvable, simplification can be made under certain circumstances. In the context of internal wave dynamics, such simplification leads to the Dubreil-Jacotin-Long (DJL) equation (Dubreil-Jacotin, 1932; Long, 1953), a single nonlinear PDE for the isopycnal displacement. The DJL equation is equivalent to the full set of stratified Euler equations, and hence its solutions are exact solitary wave solutions. In this section, we will derive the DJL equation for Boussinesq fluids in a zero background current, as this is the case that will be considered throughout the thesis. Derivations of the DJL equation for Boussinesq fluids in a sheared background current and non-Boussinesq fluids in a constant background current were presented in Stastna (2001) and Soontiens (2013).

Consider the ISW propagating horizontally with a speed $c$ in the two dimensional domain shown in figure 2.1. The domain has a finite depth with the top and bottom boundaries located at $z=0$ and $H$ (where $H<0$ ), respectively, and an infinite length in the horizontal direction. The wave is characterized by the isopycnal displacement $\eta$. An isopycnal is an isoline along which the density is constant, and the isopycnal displacement measures the displacement of an isopycnal from its undisturbed state. Suppose that the density of the fluid in the far field, denoted by $\bar{\rho}(z)$, is undisturbed and is a function of $z$ only, then the isopycnal displacement has the limiting behavior

$$
\lim _{x \rightarrow \pm \infty} \eta(x, z)=0
$$

and the density profile in the near field can be described as

$$
\rho(x, z)=\bar{\rho}(z-\eta(x, z))
$$

One of the key properties of solitary waves is that they can travel over a long distance without changing shape. In a reference frame co-moving with the wave, the flow is steady, and all dependent variables can be expressed as functions of $x-c t$ and $z$. Let $u$ be the horizontal velocity of the flow relative to $c$, and assume that $\kappa=0$, the steady-state version of the Euler equations under the Boussinesq approximation are

$$
\begin{aligned}
u u_{x}+w u_{z} & =-p_{x}, \\
u w_{x}+w w_{z} & =-p_{z}-\rho g, \\
u \rho_{x}+w \rho_{z} & =0 \\
u_{x}+w_{z} & =0
\end{aligned}
$$




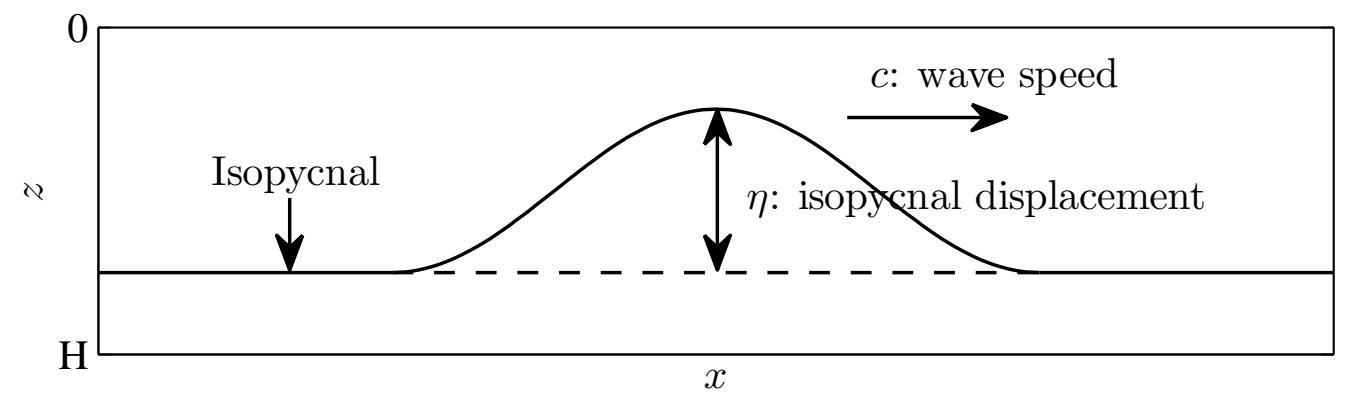

Figure 2.1: Schematic diagram of internal solitary wave propagation.

Note that this setup is equivalent to a stationary wave in a constant background current of speed $-c$. With the stream function and the vorticity field introduced in the previous section, we can rewrite equations (2.13) in the equivalent form:

$$
\begin{aligned}
\psi_{z} \omega_{x}-\psi_{x} \omega_{z} & =\rho_{x} g \\
\psi_{z} \rho_{x}-\psi_{x} \rho_{z} & =0
\end{aligned}
$$

To proceed, we shall introduce the Jacobian operator $J(a, b)$, which is defined by the determinant of the Jacobian matrix of $a$ and $b$ :

$$
J(a, b)=a_{x} b_{z}-a_{z} b_{x}
$$

It has several properties:

1. $J(a, b)=0$ implies that $a$ and $b$ are dependent;

2. for general functions $f, g$ and $u$ and constant $\lambda$,

$$
\begin{aligned}
J(f, u)+J(g, u) & =J(f+g, u), \\
J(\lambda f, u) & =\lambda J(f, u),
\end{aligned}
$$

i.e. the Jacobian operator is linear;

3. by the chain rule,

$$
\begin{aligned}
J(f(u) g, u) & =f(u) J(g, u), \\
J(g, f(u)) & =f^{\prime}(u) J(g, u)=J\left(f^{\prime}(u) g, u\right),
\end{aligned}
$$

where the primes denote the ordinary derivatives. 
Then, equations (2.14) can be further rewritten as

$$
\begin{gathered}
J\left(\nabla^{2} \psi, \psi\right)=\rho_{x} g, \\
J(\psi, \rho)=0 .
\end{gathered}
$$

The fact that $\nabla^{2} \psi=\omega$ follows from equation (2.7).

Recall from equation (2.12) that the density field can be described as a function of a single variable $(z-\eta)$. Thus, by property 3 of the Jacobi operator, equation $(2.16 \mathrm{~b})$ can be written as

$$
\bar{\rho}^{\prime}(z-\eta) J(\psi, z-\eta)=0,
$$

which implies that $\psi$ is a also function of $(z-\eta)$, assuming $\bar{\rho}^{\prime} \neq 0$ everywhere in the fluid. Note that without the presence of a background current, the velocity field has the limiting behavior

$$
\lim _{x \rightarrow \pm \infty}(u, w)=(-c, 0)
$$

which implies that

$$
\lim _{x \rightarrow \pm \infty} \psi(z-\eta)=\psi(z)=-c z .
$$

Thus, the explicit form of the stream function is

$$
\psi(z-\eta)=-c(z-\eta)
$$

With this expression, equation (2.16a) can be written as

$$
J\left(-c^{2} \nabla^{2} \eta, z-\eta\right)-\rho_{x} g=0 .
$$

Since $\rho_{x}=-J(z, \rho)=-J\left(\bar{\rho}^{\prime}(z-\eta) z, z-\eta\right)$, we can further rewrite equation (2.21) as

$$
J\left(-c^{2} \nabla^{2} \eta, z-\eta\right)+J\left(\bar{\rho}^{\prime} g z, z-\eta\right)=J\left(-c^{2} \nabla^{2} \eta+\bar{\rho}^{\prime} g z, z-\eta\right)=0,
$$

and this implies that

$$
-c^{2} \nabla^{2} \eta+\bar{\rho}^{\prime} g z=G(z-\eta)
$$

for some function $G$. The explicit form of $G$ can be determined by considering the limiting behavior $\eta=0$ as $x \rightarrow \pm \infty$ again:

$$
G(z)=\lim _{x \rightarrow \pm \infty}\left(-c^{2} \nabla^{2} \eta+\bar{\rho}^{\prime} g z\right)=\bar{\rho}^{\prime} g z,
$$


or equivalently,

$$
G(z-\eta)=\bar{\rho}^{\prime} g(z-\eta)
$$

Then, with some algebra, equation (2.23) becomes

$$
\nabla^{2} \eta+\frac{N^{2}(z-\eta)}{c^{2}} \eta=0,
$$

where $N^{2}(z-\eta)$ is the square of the buoyancy frequency.

Equation (2.26) is the DJL equation for a Boussinesq fluid with a zero background current. Even though it is much simpler than the Euler equations, it is still a fully nonlinear PDE. To the best of the author's knowledge, the DJL equation has no analytical solution, except in the special case of a linear stratification (i.e. $N^{2}$ is constant) such that equation (2.26) reduces to a linear PDE. In the present work, the DJL equation is solved numerically using the method described in Dunphy et al. (2011). 


\subsection{Numerical Method and Model Description}

Despite the fact that the DJL equation simplifies the full set of Euler equations to a single PDE while preserving the nonlinearity of fluid flows, it also poses its own challenges. First of all, the DJL equation is derived from the steady-state theory, which does not describe any temporal variation in the fluid flows. Second, the DJL theory is limited to inviscid flows, and hence it only applies to flows away from the boundary layer but features relevant to boundary layer instabilities are absent. For these reasons, the numerical simulations presented in this study employ the full set of the stratified incompressible Navier-Stokes equations under the Boussinesq approximation, whereas the solutions of the DJL equation are used to initialize the numerical simulations, i.e. as the initial conditions only.

The numerical model SPINS is developed as a PhD project by Christopher Subich. While this section only provides a brief introduction, a complete description of the model can be found in his thesis (Subich, 2011) and a companion paper (Subich et al., 2013), where a detailed validation and accuracy analysis through several test cases is also given. The model employs a three dimensional spectral collocation method, which yields highly accurate results at moderate grid resolutions. One of the key features of this model is the grid mapping technique, which allows problems with irregular (though relatively smooth) bottom topography to be solved by transforming the physical domain into a rectangular computational domain. The spatial discretization is implemented with both the Fourier expansion and the Chebyshev polynomial as appropriate for boundary conditions and domain mapping. In the vertical direction, the Chebyshev discretization is applied if noslip boundary conditions or coordinate mapping is used. The Chebyshev points are defined by (Trefethen, 2000, chapter 5)

$$
\xi_{j}=\cos \left(\frac{\pi j}{N}\right), \quad j=0,1, \ldots, N
$$

on the computational grid $\xi \in[-1,1]$, where $N$ is the number of grid points. The Chebyshev discretization does not only avoid the Runge phenomenon commonly found in polynomial interpolations using equally spaced grid, but also naturally clusters points in the boundary layers where instabilities are more likely to develop. In the horizontal directions, the choice of spectral expansion is more flexible. It can be the Fourier discretization for periodic boundary conditions, the sine/cosine discretization for free-slip boundary conditions, or the Chebyshev polynomials for no-slip boundary conditions. For time stepping, the model uses the adaptive third-order Adams-Bashforth method. Compared with other lower order methods, it requires little additional computational cost but significantly increases the accuracy. 


\subsection{Shear Instability}

Stratified shear instability occurs when there is sufficient horizontal velocity shear in a stratified fluid. It is characterized by the Kelvin-Helmholtz billows growing in the shear layer, and is also commonly called the Kelvin-Helmholtz instability. It is ubiquitous in the atmosphere and the ocean, and is easy to generate in the laboratory using a tilted tube (e.g. Thorpe, 1971). This section will provide the inviscid linear stability analysis leading to the Taylor-Goldstein (TG) equation (Miles, 1961; Drazin and Howard, 1966) and the criteria for determining the linear stability. The influence of viscosity on the stability of a stratified shear flow is beyond the scope of this study, an investigation on this topic is provided in Defina et al. (1999).

Consider the two dimensional inviscid stratified parallel shear flow whose background profiles of the horizontal velocity $U(z)$ and density $\bar{\rho}(z)$ are illustrated in figure 2.2 . The total velocity, density and pressure profiles are given by

$$
\begin{aligned}
\boldsymbol{u} & =(U(z), 0)+\epsilon(u, w), \\
\rho & =\bar{\rho}(z)+\epsilon \rho^{\prime} \\
p & =p_{H}(z)+\epsilon p^{\prime}
\end{aligned}
$$

respectively, where $p_{H}(z)$ is the hydrostatic pressure and the $\mathcal{O}(\epsilon)$ terms represent perturbations to the background states ( $\epsilon$ is an infinitesimally small parameter). Then, the total vorticity $\omega$ can be expressed as

$$
\omega=U_{z}+\epsilon \omega^{\prime}
$$

where $U_{z}$ is the background vorticity and $\omega^{\prime}=u_{z}-w_{x}$ is the perturbation vorticity.

Under the Boussinesq approximation, the $\mathcal{O}(\epsilon)$ equations of the Euler equations (assuming $\kappa=0$ ) are given by

$$
\begin{aligned}
u_{t}+U u_{x}+w U_{z} & =-p_{x}^{\prime}, \\
w_{t}+U w_{x} & =-p_{z}^{\prime}-\rho^{\prime} g, \\
\rho_{t}^{\prime}+U \rho_{x}^{\prime}+w \bar{\rho}_{z} & =0 \\
u_{x}+w_{z} & =0 .
\end{aligned}
$$

The $\mathcal{O}(\epsilon)$ vorticity equation, obtained by taking the curl of the momentum equations, is

$$
\omega_{t}^{\prime}+U \omega_{x}^{\prime}+w U_{z z}=\rho_{x}^{\prime} g
$$



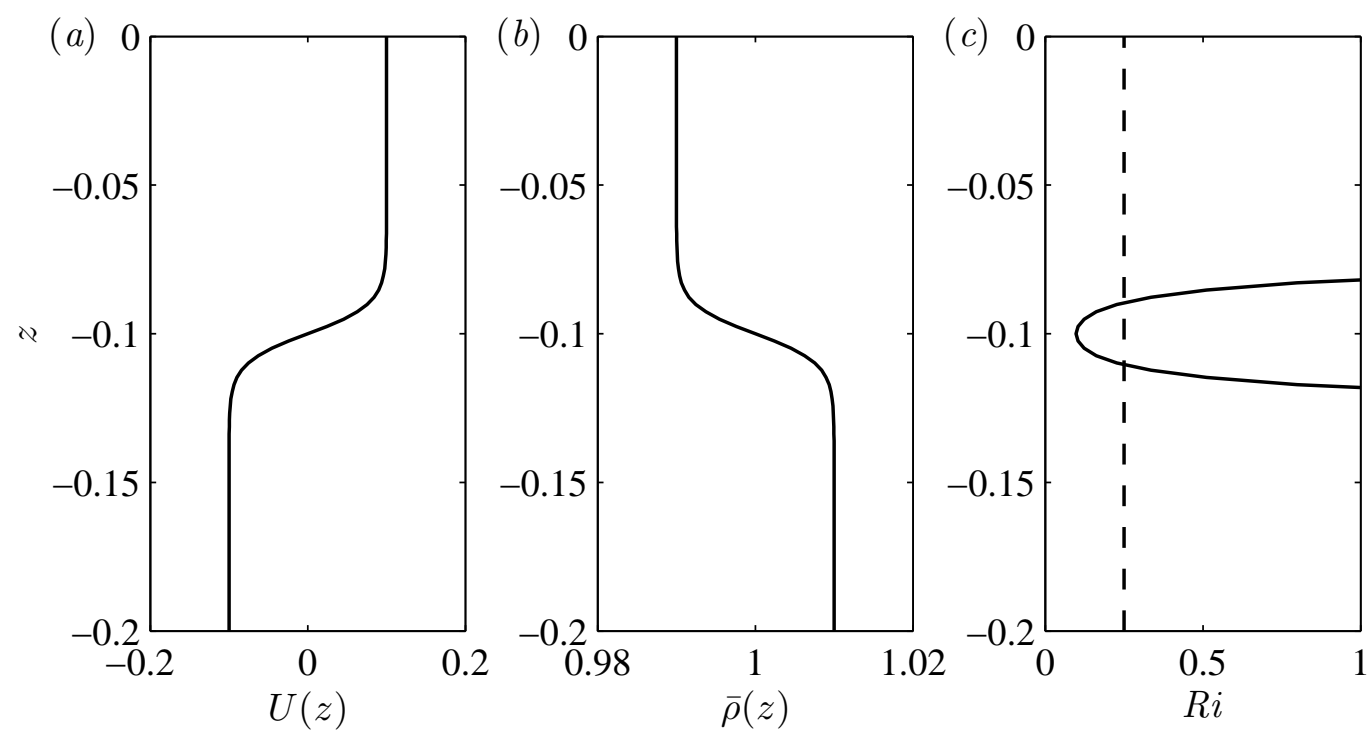

Figure 2.2: Background profiles of $(a)$ the horizontal velocity and $(b)$ the density for a two dimensional inviscid stratified parallel shear flow. The resulting Richardson number is shown in $(c)$ with the dashed line indicating the critical Richardson number $R i=0.25$.

Here, the term $\omega_{t}^{\prime}$ represents the rate of change of vorticity in time, $U \omega_{x}^{\prime}$ represents the horizontal advection of the perturbation vorticity, and $w U_{z z}$ represents the vertical advection of the background vorticity. Equation (2.31) states that the sum of these physical quantities is balanced by the baroclinic vorticity production $\rho_{x}^{\prime} g$.

If we define the stream function from the perturbation velocity such that $(u, w)=$ $\left(\psi_{z},-\psi_{x}\right)$ and $\omega^{\prime}=\nabla^{2} \psi$, then equations (2.30c) and (2.31) can be rewritten as

$$
\begin{aligned}
\rho_{t}^{\prime}+U \rho_{x}^{\prime}+\psi_{x} \frac{N^{2}}{g} & =0, \\
\nabla^{2} \psi_{t}+U \nabla^{2} \psi_{x}-\psi_{x} U_{z z} & =\rho_{x}^{\prime} g,
\end{aligned}
$$

where $N^{2}$ is the squared buoyancy frequency. We shall adopt the method of normal modes (Kundu et al., 2012, section 11.2) by assuming plane wave solutions of the forms

$$
\begin{aligned}
& \rho^{\prime}(x, z, t)=d(z) e^{i k(x-c t)}, \\
& \psi(x, z, t)=\phi(z) e^{i k(x-c t)},
\end{aligned}
$$

where $\phi(z)$ is the vertical structure of waves of disturbance. To investigate the temporally growing instability, we assume that the wave number $k$ is real and known, and the phase 
speed $c$ is complex and unknown (here $c$ is not the ISW propagation speed). Substituting the normal modes into equations (2.32) and canceling the common factors, we obtain

$$
\begin{aligned}
-c d+d U+\phi \frac{N^{2}}{g} & =0, \\
-c\left(\phi_{z z}-k^{2} \phi\right)+U\left(\phi_{z z}-k^{2} \phi\right)-U_{z z} \phi & =d g .
\end{aligned}
$$

These two equations can be further rearranged and combined together, in a process that yields the TG equation

$$
(U-c)\left(\phi_{z z}-k^{2} \phi\right)-\phi U_{z z}+\frac{\phi N^{2}}{U-c}=0 .
$$

The boundary conditions for the TG equation are the no normal flow conditions given by

$$
\phi(H)=\phi(0)=0 .
$$

The TG equation governs the behavior of perturbations in a stratified parallel shear flow and provides some general stability criteria. The stability of the flow is determined by $c$ such that, if the imaginary part of $c$ is positive, then the solutions of $\rho^{\prime}$ and $\psi$ will grow exponentially with respect to time, until the nonlinear effect becomes dominant. A well known criterion for determining the linear stability of an inviscid stratified flow is the Richardson number (sometimes called the gradient Richardson number), defined by

$$
R i=\frac{N^{2}}{U_{z}^{2}} .
$$

It measures the ratio between the strength of the stratification and the shear stress. A sufficient condition for the flow to be linearly stable is that the local Richardson number exceed 0.25 throughout the flow (Miles, 1961; Howard, 1961), but $R i<0.25$ does not mean the flow is necessarily unstable (the criterion is not sufficient). When the flow is not a parallel shear flow, the meaning of $R i$ is not clear. Note that the instability may also grow spatially, provided that $k$ is complex and the imaginary part of $k$ is negative.

\subsubsection{Numerical solutions}

We shall solve the TG equation numerically using the Chebyshev polynomials. Consider equations (2.34) rewritten in the equivalent form

$$
\begin{aligned}
N^{2} \phi(z)+U g d(z) & =c g d(z), \\
{\left[U\left(\partial_{z}^{2}-k^{2}\right)-U_{z z}\right] \phi(z)-g d(z) } & =c\left(\partial_{z}^{2}-k^{2}\right) \phi(z),
\end{aligned}
$$


and the grid mapping defined by

$$
z=\frac{1}{2} H(1-\xi) \quad \Longleftrightarrow \quad \xi=1-\frac{2}{H} z
$$

where $z \in[H, 0]$ and $\xi \in[-1,1]$. Let $D^{2}$ be the second order Chebyshev differentiation matrix (Trefethen, 2000, chapter 6) with the first and last columns and rows removed (since we have homogeneous Dirichlet boundary conditions here and thus these entries do not contribute to the final solutions), and $I$ be the identity matrix, we can write the Chebyshev discretization of the linear operator $\left(\partial_{z}^{2}-k^{2}\right)$ in equation $(2.38 \mathrm{~b})$ as

$$
L=\frac{4}{H^{2}} D^{2}-k^{2} I .
$$

The coefficient of $D^{2}$ follows from the fact that

$$
\partial_{z}=\xi_{z} \partial_{\xi}=-\frac{2}{H} \partial_{\xi}
$$

implied in the mapping (2.39). Suppose other variables are also evaluated at the Chebyshev points $\xi_{j}$ defined in (2.27), then the Chebyshev discretization of equations (2.38) becomes a single eigenvalue problem, whose matrix form is given by

$$
\left(\begin{array}{cc}
\boldsymbol{N}^{2} & \boldsymbol{U} \\
\boldsymbol{U} L-\boldsymbol{U}_{\boldsymbol{z} \boldsymbol{z}} & -I
\end{array}\right)\left(\begin{array}{c}
\phi \\
g d
\end{array}\right)=c\left(\begin{array}{cc}
\mathbf{0} & I \\
L & \mathbf{0}
\end{array}\right)\left(\begin{array}{c}
\phi \\
g d
\end{array}\right)
$$

where $\mathbf{0}$ denotes the zero matrix, and bold symbols denote the square diagonal matrices with the elements of the corresponding vectors on the main diagonal.

As an example, consider the background velocity and density profiles given by

$$
\begin{aligned}
& U(z)=0.1 \tanh \left(\frac{z-0.5 H}{0.01}\right), \\
& \bar{\rho}(z)=1+0.01 \tanh \left(\frac{0.5 H-z}{0.01}\right),
\end{aligned}
$$

as plotted in figure 2.2. Note that here the bottom boundary is located at $H=-0.2$, and the density profile implies that the pycnocline is located at $z=0.5 H$ and has a thickness of 0.01 . The corresponding Richardson number plot suggests that the instability may be generated at the shear layer located at $z=-0.1$.

To find the fastest growing wave of disturbance, 30 different wavelengths ranging from 0.1 to 0.35 are tested. The wave number $k$ is defined from the wavelength $\lambda$ such that

$$
k=\frac{2 \pi}{\lambda} .
$$



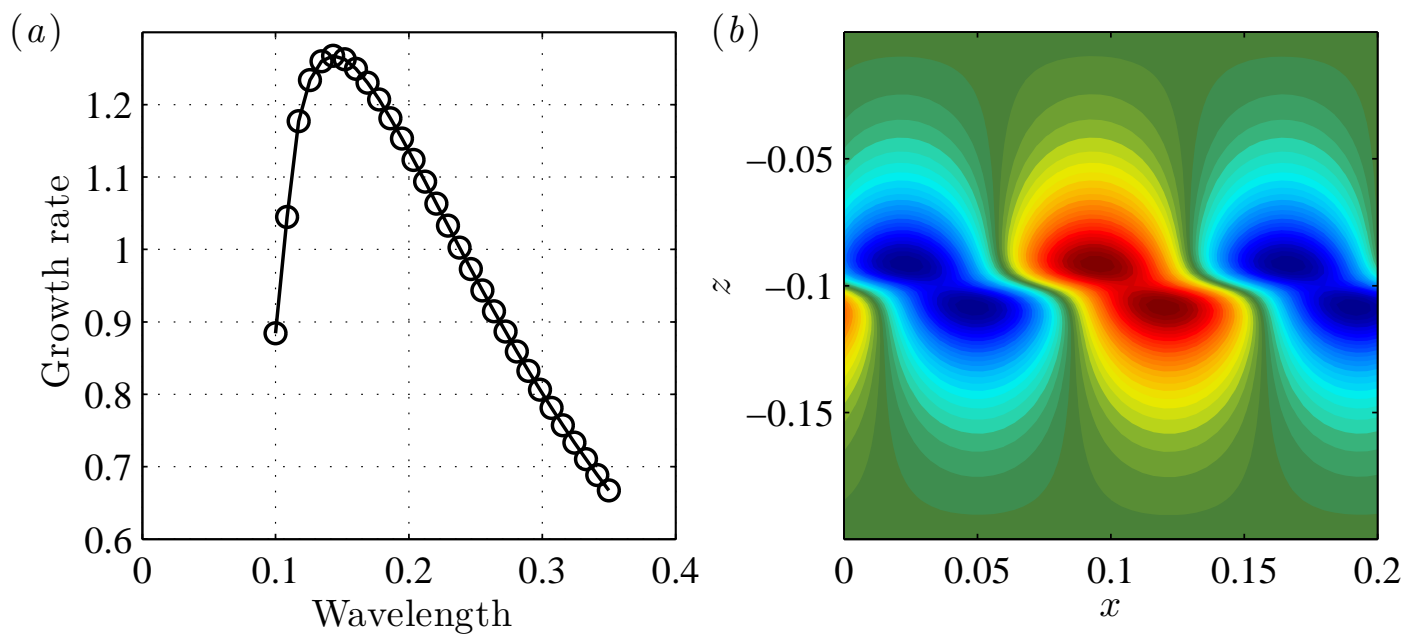

Figure 2.3: Perturbation profiles of the shear flow. Panel $(a)$ : growth rate of waves of disturbance versus wave length. Panel $(b)$ : real part of the stream function corresponding to the fastest growing wave, determined by equation (2.46).

The growth rate is determined by $c_{i} k$ where $c_{i}$ is the imaginary part of the eigenvalue $c$. For each wavelength, only the eigenvalue with the largest imaginary part is relevant in determining the largest growth rate. These growth rates are plotted against their wavelengths in figure $2.3(\mathrm{a})$, from which the wavelength that yields the fastest growing wave can be determined. Assuming a steady state solution (i.e. $t=0$ ), the real part of the stream function (2.33b) for this fastest growing wave is given by

$$
\Re(\psi)=\phi_{r} \cos \left(k^{*} x\right)-\phi_{i} \sin \left(k^{*} x\right),
$$

where $k^{*}$ is the wavenumber of the fastest growing wave and $\phi_{r}$ and $\phi_{i}$ are the real and imaginary parts of the vertical structure function $\phi(z)$, respectively. This quantity is plotted as filled contours in the two dimensional domain shown in figure $2.3(b)$, where a horizontal shear layer can be clearly seen at $z=-0.1$. 


\subsection{Bottom Shear Stress}

An interesting and important feature of shoaling ISWs is that they can interact with the bottom boundary, resulting in boundary layer instabilities and material mixing. The bottom shear stress provides a useful diagnostic in the analysis of such interaction. This section will derive the formula for determining the shear stress on bottom boundaries with relatively smooth topography using the Cauchy's stress theorem.

The Cauchy's theorem (Kundu et al., 2012) states that the traction force $\boldsymbol{t}$ on a surface (i.e. the bottom boundary) due to fluid motion is given by

$$
\boldsymbol{t}(\boldsymbol{x} ; \hat{n})=\boldsymbol{\tau}(\boldsymbol{x}) \hat{n}
$$

where

$$
\boldsymbol{\tau}=\left(\begin{array}{ccc}
2 \mu u_{x}-p & \mu\left(u_{y}+v_{x}\right) & \mu\left(u_{z}+w_{x}\right) \\
\mu\left(u_{y}+v_{x}\right) & 2 \mu v_{y}-p & \mu\left(v_{z}+w_{y}\right) \\
\mu\left(u_{z}+w_{x}\right) & \mu\left(v_{z}+w_{y}\right) & 2 \mu w_{z}-p
\end{array}\right)
$$

is the Cauchy stress tensor for incompressible fluids (Kundu et al., 2012, equation 4.35), and $\hat{n}$ is the unit normal vector. The shear stress, denoted by $\boldsymbol{t}_{s}$, is defined by the tangential component of the traction force:

$$
\boldsymbol{t}_{s}=\left(t_{x}, t_{y}\right)=\boldsymbol{t} \cdot\left(\hat{s}_{x}, \hat{s}_{y}\right),
$$

where $t_{x}$ and $t_{y}$ are directed along the streamwise and spanwise directions, respectively, and $\hat{s}_{x}$ and $\hat{s}_{y}$ are the unit normal vectors. Note that although the subscript $x$ is used here, $\hat{s}_{x}$ is not necessarily parallel to the $x$-axis if bottom topography is present in the $x$ direction. For example, suppose the bottom topography is specified by $z=H(x)$ for some function $H$, then these unit vectors are given by

$$
\hat{n}=\frac{1}{\sqrt{1+H^{\prime}(x)^{2}}}\left[\begin{array}{c}
-H^{\prime}(x) \\
0 \\
1
\end{array}\right], \quad \hat{s}_{x}=\frac{1}{\sqrt{1+H^{\prime}(x)^{2}}}\left[\begin{array}{c}
1 \\
0 \\
H^{\prime}(x)
\end{array}\right], \quad \text { and } \quad \hat{s}_{y}=\left[\begin{array}{l}
0 \\
1 \\
0
\end{array}\right] \text {, }
$$

and the resulting bottom shear stress can be written explicitly as

$$
\begin{aligned}
& t_{x}(x, y, H(x))=\frac{\mu}{1+H^{\prime}(x)^{2}}\left[2 H^{\prime}(x)\left(w_{z}-u_{x}\right)+\left(1-H^{\prime}(x)^{2}\right)\left(u_{z}+w_{x}\right)\right], \\
& t_{y}(x, y, H(x))=\frac{\mu}{1+H^{\prime}(x)^{2}}\left[v_{z}-v_{x} H^{\prime}(x)\right] .
\end{aligned}
$$


Note that since the no-flux and no-slip boundary conditions are used throughout this thesis and the bottom topography is a function of $x$ only, all $y$-derivatives of the velocity field are necessarily zero along the bottom boundary and are thus dropped in equation (2.51). When the bottom boundary is flat, we have $H^{\prime}(x)=0$ and $w_{x}=0$, and hence equations (2.51) reduce to

$$
t_{x}=\mu u_{z}, \quad \text { and } \quad t_{y}=\mu v_{z} .
$$

Also note that in two dimensions, since there is no $y$-velocity, the bottom shear stress is determined by equation (2.51a) only.

We shall mention that it is extremely expensive to compute the entire derivative field of $\boldsymbol{u}$ in three dimensions. Nevertheless, equations (2.51) suggest that, without spanwise variation of the topography, the bottom shear stress is independent of any $y$-derivatives of the velocity field. Hence, for each grid point in the $y$-direction, only the two-dimensional derivative field on the $x-z$ plane is relevant. Taking advantage of this fact, an algorithm has been developed to solve for the bottom shear stress as a two-dimensional problem recursively. Although it may be slower, compared with the method that tries to compute the entire derivative field it is much more memory efficient and computationally stable. This technique will be used in the remaining chapters to analyze the evolution of bottom shear stress due to wave passage, as well as the wave-induced instabilities. 


\section{Chapter 3}

\section{Model Setup and Two-Dimensional Simulations}

The breaking of nonlinear internal waves and the formation of trapped cores has been considered by a number of authors using numerical simulations, laboratory experiments and field measurements. While the majority of past work has examined waves of depression (e.g. Lamb, 2002, 2003; Aghsaee et al., 2012; Carr et al., 2012) as they are commonly observed in oceans and deep lakes, waves of elevation, which occur when the pycnocline is below the mid-depth, are more typical of shallow waters such as near-coastal regions (e.g. Klymak and Moum, 2003; Scotti and Pineda, 2004). In these waves, a trapped core is formed close to the ocean bottom and interacts directly with the bottom boundary layer. For this reason, waves of elevation are expected to provide a more effective mechanism for material mixing and sediment resuspension than waves of depression.

In the present work, numerical simulations of laboratory-scale ISWs of elevation shoaling onto a small-amplitude shelf are performed in both two- and three-dimensions. This chapter focuses on the two-dimensional simulations. The mechanism responsible for the generation of instabilities during the waves' shoaling is examined through the variation of stratification. The behavior of the shoaling wave over bottom topography with short length scale undulations is also studied. In particular, four cases with different stratification and bottom topography are discussed in detail. Before presenting the simulation results, the model setup and parameter space is outline in a separate section. 


\subsection{Model Setup and Simulation Parameters}

The two-dimensional (henceforth 2D) simulations are performed in a mapped rectangular domain with an overall length $L_{x}=7 \mathrm{~m}$ and a depth $L_{z}=0.2 \mathrm{~m}$. The surface of the fluid is located at $z=0$, and the bottom boundary is chosen to represent an asymmetric, small amplitude shelf, which has a maximum height $\Delta H=0.025 \mathrm{~m}$ located at $x_{c}=6 \mathrm{~m}$. The functional form of the bottom topography is given by the skewed Gaussian distribution

$$
H(x)=-L_{z}+\Delta H e^{-h(x)}[1+\epsilon(x)],
$$

where $h(x)$ is defined by

$$
h(x)= \begin{cases}{\left[0.5\left(x-x_{c}\right)\right]^{2},} & \text { if } x \leq x_{c} \\ {\left[0.5\left(x-x_{c}\right)\right]^{2}+0.5\left[2\left(x-x_{c}\right)\right]^{8},} & \text { if } x>x_{c}\end{cases}
$$

and $\epsilon(x)$ is either zero or $0.1 \sin (20 \pi x)$ depending on the smoothness of the bottom boundary. For all simulations, periodic boundary conditions are used in the $x$-direction, and no-flux and no-slip boundary conditions are used in the $z$-direction. As appropriate for these boundary conditions, the Fourier series expansion is used in the $x$ direction and the Chebyshev discretization is applied in the $z$ direction. The grid size, denoted by $N_{x} \times N_{z}$, is $6144 \times 256$. This gives a constant horizontal grid spacing of about $1 \mathrm{~mm}$ and a varying vertical grid spacing of less than $2 \mathrm{~mm}$ at the mid-depth with clustering near the boundaries, so that any instabilities along the bottom boundary can be well resolved.

We focus on flows in a quasi two-layer stratification with a dimensionless density difference $\Delta \rho=0.02$, for which the Boussinesq approximation can be adopted. The background density profile, non-dimensionalized by the reference density $\rho_{0}=10^{3} \mathrm{~kg} / \mathrm{m}^{3}$, is given by

$$
\bar{\rho}(z)=1-0.5 \Delta \rho \tanh \left(\frac{z-z_{0}}{0.5 d}\right)
$$

where $z_{0}$ is the location of the pycnocline, and $d$ is the thickness of the pycnocline. For the $2 \mathrm{D}$ simulations, $d$ is fixed at $0.02 \mathrm{~m}$, while three values of $z_{0}$ are used: $-0.1725 \mathrm{~m}$, $-0.16 \mathrm{~m}$ and $-0.15 \mathrm{~m}$. The parameter space is summarized in table 3.1. The viscosity and diffusivity are fixed at $\nu=\kappa=10^{-6} \mathrm{~m}^{2} / \mathrm{s}$, which give a Schmidt number $S c=1$. The Schmidt number, defined by $S c=\nu / \kappa$, measures the ratio between the momentum diffusivity (i.e. viscosity) and the mass diffusivity.

Each simulation is initialized with a single mode- 1 solitary wave, specified by interpolating a solution of the DJL equation onto the left half of the domain (i.e., from $x=0$ to 


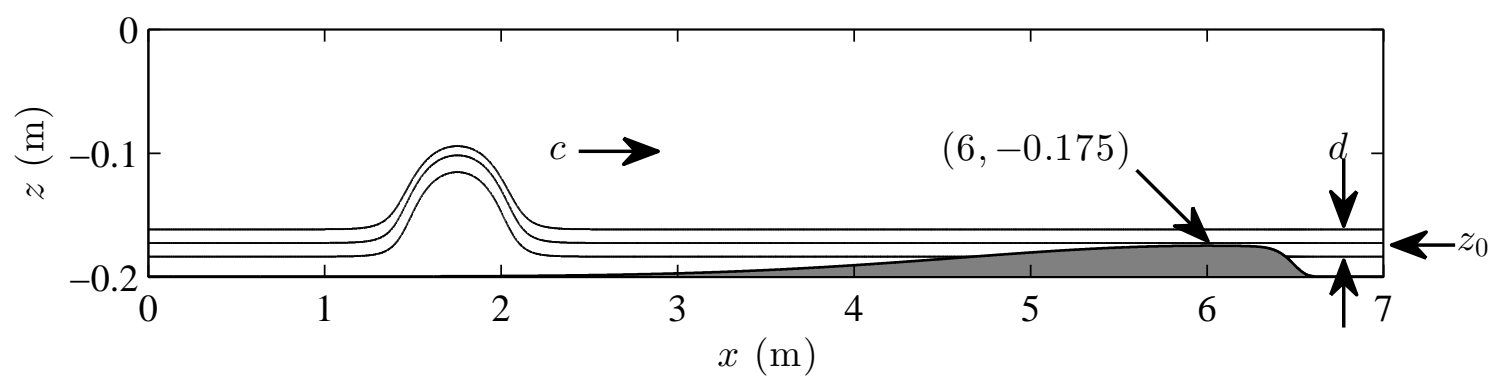

Figure 3.1: Schematic diagram showing the computational domain and model setup. The origin is fixed at the upper left corner of the domain, and the unit for both axes is meter. The bottom topography is given by equation (3.1) with the crest located at $(x, z)=$ $(6,-0.175) \mathrm{m}$. The pycnocline is centered at $z=z_{0} \mathrm{~m}$ and has a thickness $d=0.02 \mathrm{~m}$. The initial wave propagates to the right at a speed $c$.

\begin{tabular}{lccccccc}
\hline Simulation & Topography & Pycnocline & \multicolumn{5}{c}{ Wave Parameters } \\
& $\epsilon(x)$ & $z_{0}$ & $\eta_{\max }$ & $c$ & $u_{\min }$ & $u_{\max }$ & $w_{\max }$ \\
& & $(\mathrm{m})$ & $(\mathrm{cm})$ & $(\mathrm{cm} / \mathrm{s})$ & $(\mathrm{cm} / \mathrm{s})$ & $(\mathrm{cm} / \mathrm{s})$ & $(\mathrm{cm} / \mathrm{s})$ \\
\hline Base Case & 0 & -0.1725 & 6.69 & 9.26 & -6.85 & 8.17 & 2.83 \\
Medium & 0 & -0.16 & 5.95 & 9.35 & -5.78 & 6.50 & 1.83 \\
Higher & 0 & -0.15 & 5.09 & 9.38 & -5.01 & 5.38 & 1.23 \\
Rough & $0.1 \sin (20 \pi x)$ & -0.16 & 5.95 & 9.35 & -5.78 & 6.50 & 1.83 \\
\hline
\end{tabular}

Table 3.1: List of parameters for $2 \mathrm{D}$ simulations, where $\eta_{\max }$ is the maximum isopycnal displacement which measures the wave amplitude, and $c$ is the ISW propagation speed. The minimum values of $w$ is equal to $-w_{\max }$ by symmetry. All wave parameters are obtained from solutions of the DJL equation and are for the initial waves only (i.e. at $t=0 \mathrm{~s}$ ). Parameters fixed for all simulations are not shown here.

$3.5 \mathrm{~m})$. A schematic diagram of the model setup showing the initial wave and the bottom topography is given in figure 3.1. The Reynolds number, based on the channel half-depth and the wave propagation speed (see table 3.1), is approximately $R e=10^{4}$. Also shown in table 3.1 is that, in all simulations, the maximum horizontal velocity $u_{\max }$ is less than the wave propagation speed. According to the classification of ISW upper bounds in Lamb (2003), this suggests that none of the initial waves reach the breaking limit and thus no trapped core is initially present. 


\subsection{The Base Case}

In the basic $2 \mathrm{D}$ case, the center of the pycnocline is located at $z_{0}=-0.1725 \mathrm{~m}$. Note that since the crest of the bottom topography is located at $z=-0.175 \mathrm{~m}$ and the width of the pycnocline is $d=0.02 \mathrm{~m}$, the pycnocline in this case intercepts with the bottom boundary. An impression of the overall flow structure in the full domain can be gained from figure 3.2 , though these plots do not represent the true aspect ratio because the horizontal length scale of the model is much larger than the vertical length scale. Panel $(a)$ shows that shape of the initial wave is symmetric about its crest. However, as it propagates and shoals onto the shelf, the wave becomes narrower and loses the symmetry, as shown in panel $(b)$. The loss of symmetry takes place in a way such that the pycnocline at the front of the wave gets squeezed together. This results in a sharp transition zone in the density field, in which instabilities are likely to be generated.
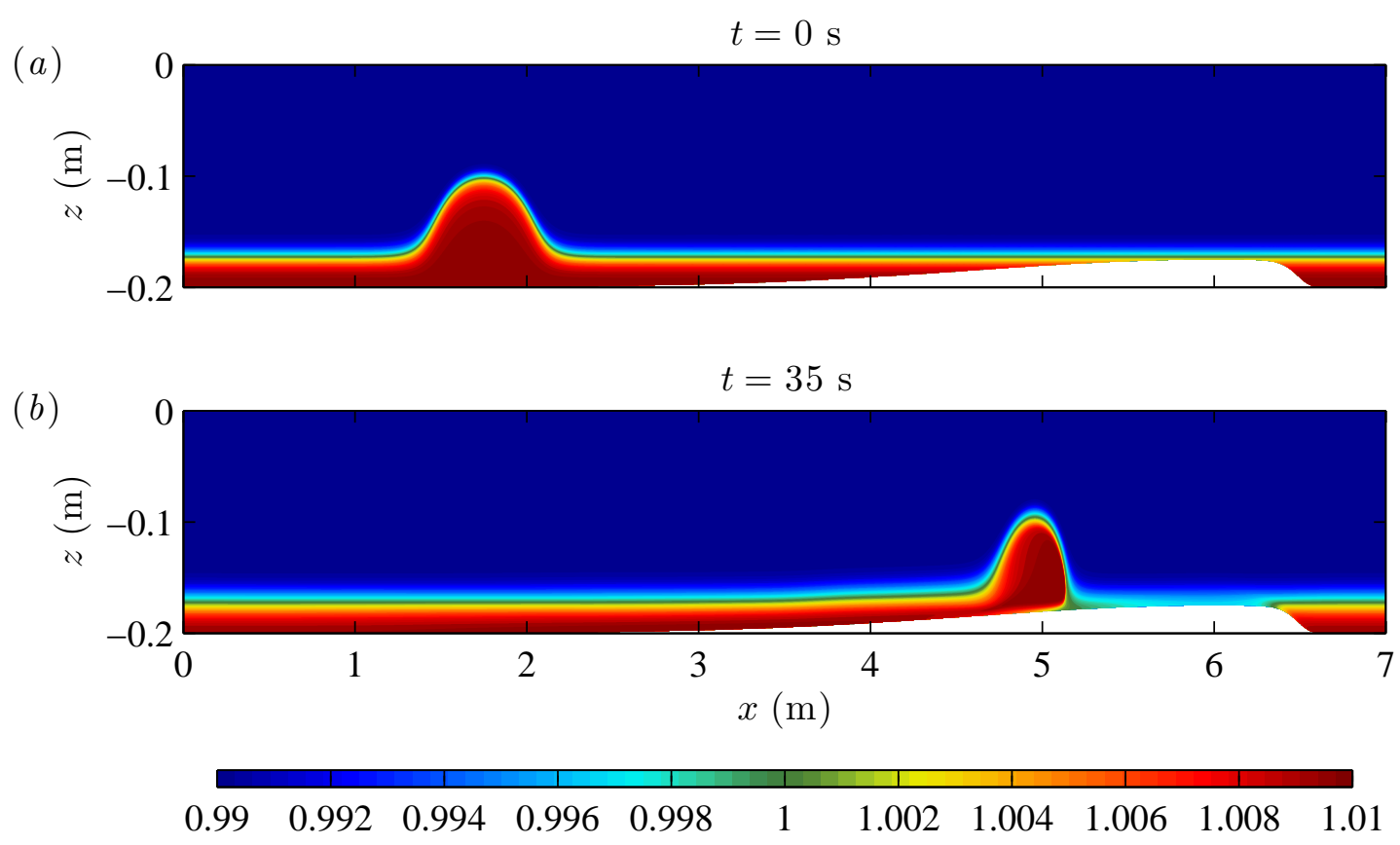

Figure 3.2: Normalized density contours showing $(a)$ the initial wave and $(b)$ its propagation and shoaling. Here and henceforth, the color axes of all density plots are saturated at $\rho=1 \pm 0.01$, with dense fluids shown in red, light fluid shown in blue and midrange values shown in green. 


\subsubsection{Shear Instability}

Although the amplitude of the wave is large $\left(\eta_{\max }=6.69 \mathrm{~cm}\right.$, or approximately $1 / 3$ of the channel depth), the wave is still broad due to the aspect ratio. Thus, in the center of the wave, the flow is nearly horizontal. As the wave shoals, the maximum horizontal velocity in the center of the wave exceeds the wave propagation speed $\left(u_{\max }=10.16 \mathrm{~cm} / \mathrm{s}\right.$ at $t=35$ $\mathrm{s}, u_{\max }=11.53 \mathrm{~cm} / \mathrm{s}$ at $t=40 \mathrm{~s}$, whereas the ISW propagation speed is $c=9.26 \mathrm{~cm} / \mathrm{s}$ ), increasing the shear stress at the edge of the wave and hence, along with any imposed noise, providing the trigger for shear instability.
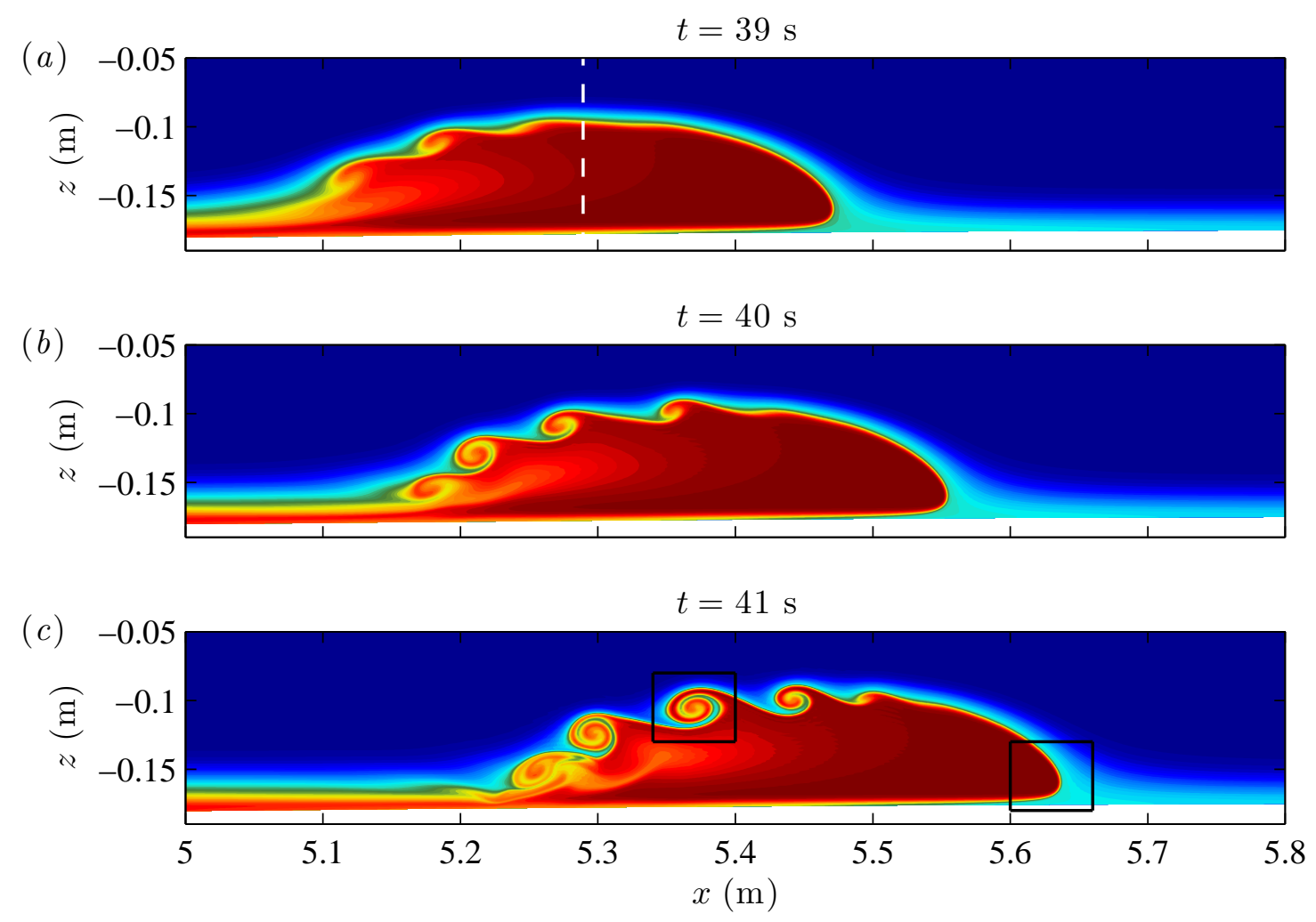

Figure 3.3: Three panels of shaded density contours showing the propagation and shoaling of the wave and the development of the core at $(a) t=39 \mathrm{~s},(b) t=40 \mathrm{~s}$ and $(c) t=41 \mathrm{~s}$. The dashed line in panel $(a)$ is located at $x=5.29 \mathrm{~m}$, along which the analysis of shear instability is given in figure 3.5. Details of the billow and the wave front as indicated by the boxes in panel $(c)$ are plotted in figure 3.4. 

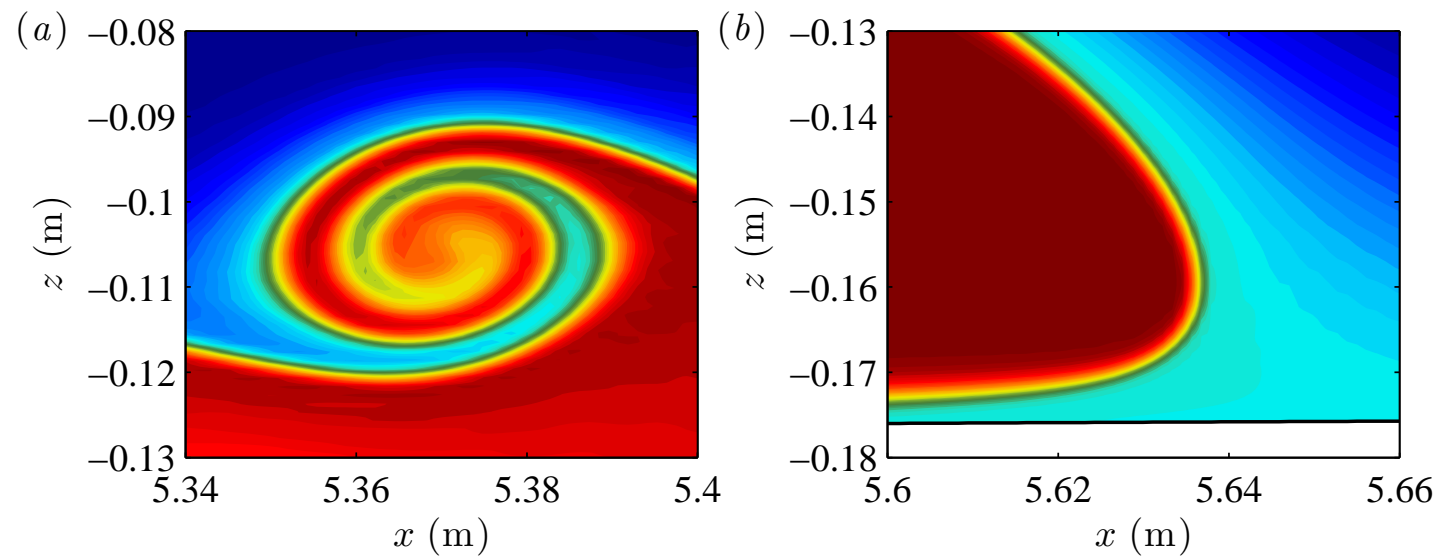

Figure 3.4: Detailed density contours of $(a)$ one of the billows at the edge of the core and (b) the wave front and the bottom boundary layer at $t=41 \mathrm{~s}$. See figure $3.3(c)$.

Detailed density contours showing the evolution of the shoaling wave are given in figure 3.3. This figure suggests that, during shoaling, the wave breaks and begins to form a recirculating core. In panel $(a)$, the core is beginning to overturn toward the left, while a high shear region is developing at the edge of the core, yielding Kelvin-Helmholtz billows that grow as they propagate in the downstream direction (in a reference frame moving with the wave). In panel (b), these billows have reached their full size, while in panel $(c)$ the initial billows have begun to be swept into the bottom boundary layer (BBL), enhancing bottom shear stress and thereby possibly contributing to sediment resuspension. At the same time, further billows have begun to grow on the high shear edge of the core. The detail of one of these billows is shown in figure $3.4(a)$.

We shall analyze the instability using the method outlined in section 2.4. In particular, the analysis is performed along the vertical slice located at $x=5.29 \mathrm{~m}$ at $t=39 \mathrm{~s}$, as indicated by the dashed line figure $3.3(a)$. This line corresponds to the location of the wave crest, where the flow is parallel and nearly horizontal across the whole vertical column. The background horizontal velocity profile is plotted in figure $3.5(a)$, where a shear layer can be clearly seen at $z \approx-0.1 \mathrm{~m}$. Note that although velocity shear is also found along the top and bottom boundaries due to the no-slip condition, no instability is observed in these areas. Since the pycnocline is located at $z \approx-0.1 \mathrm{~m}$, large buoyancy frequency is also found in this shear layer, as shown in figure $3.5(b)$. Therefore, the background flow can be considered as a typical stratified shear flow. The minimum local Richardson number found in this shear layer is $R i=0.065$, much smaller than the critical Richardson number 

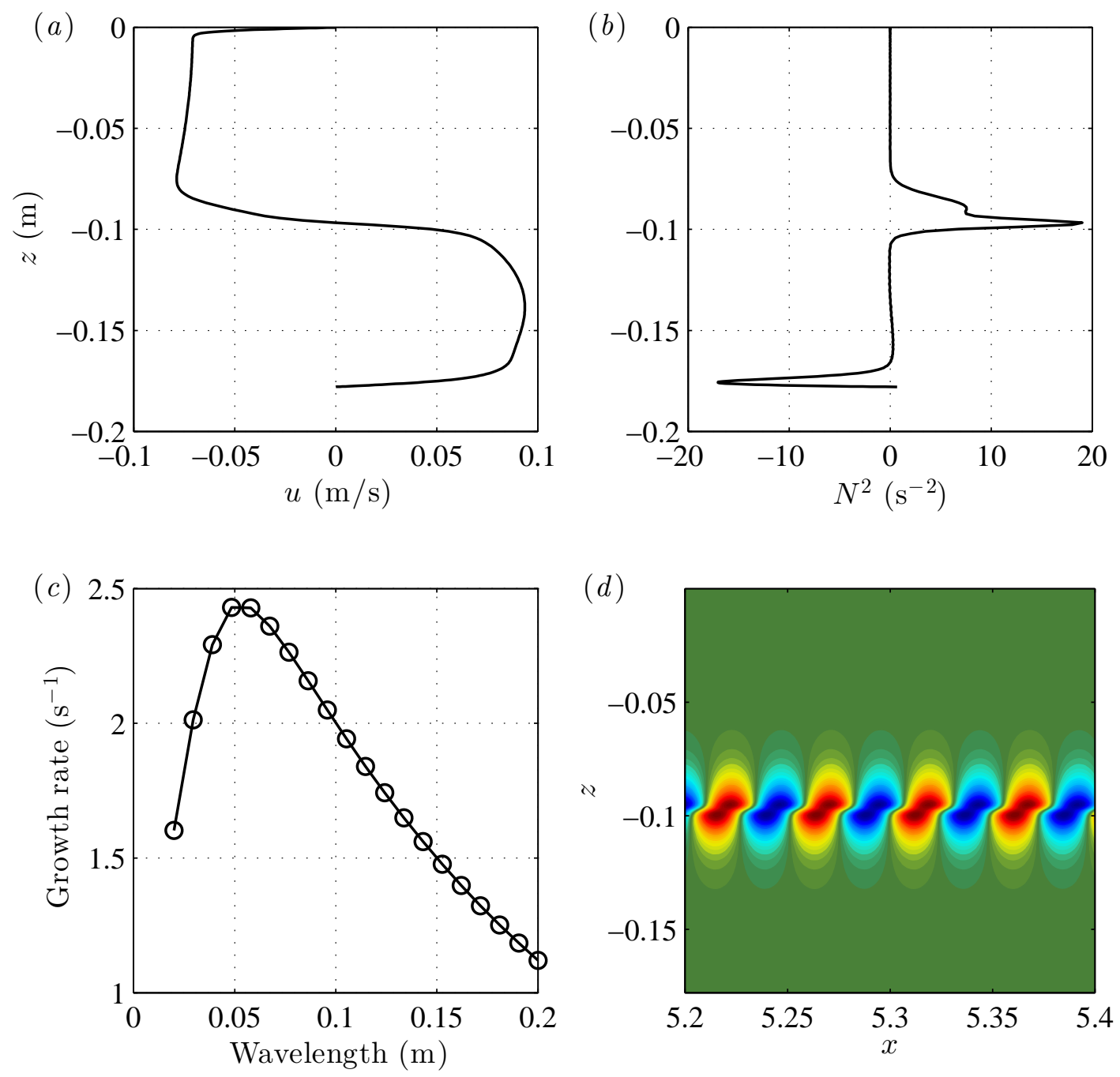

Figure 3.5: Analysis of shear instability along $x=5.29 \mathrm{~m}$ at $t=39 \mathrm{~s}$ (the dashed line in figure $3.3(a)$ ). Panel $(a)$ : background horizontal velocity profile. Panel $(b)$ : squared buoyancy frequency, where the negative value at $z \approx-0.175 \mathrm{~m}$ is due to the no-slip boundary condition (see figure 3.4 (b)). Panel $(c)$ : growth rate of waves of disturbance. Panel $(d)$ : filled contour plot of the stream function showing the 2D structure of the fastest growing wave. 

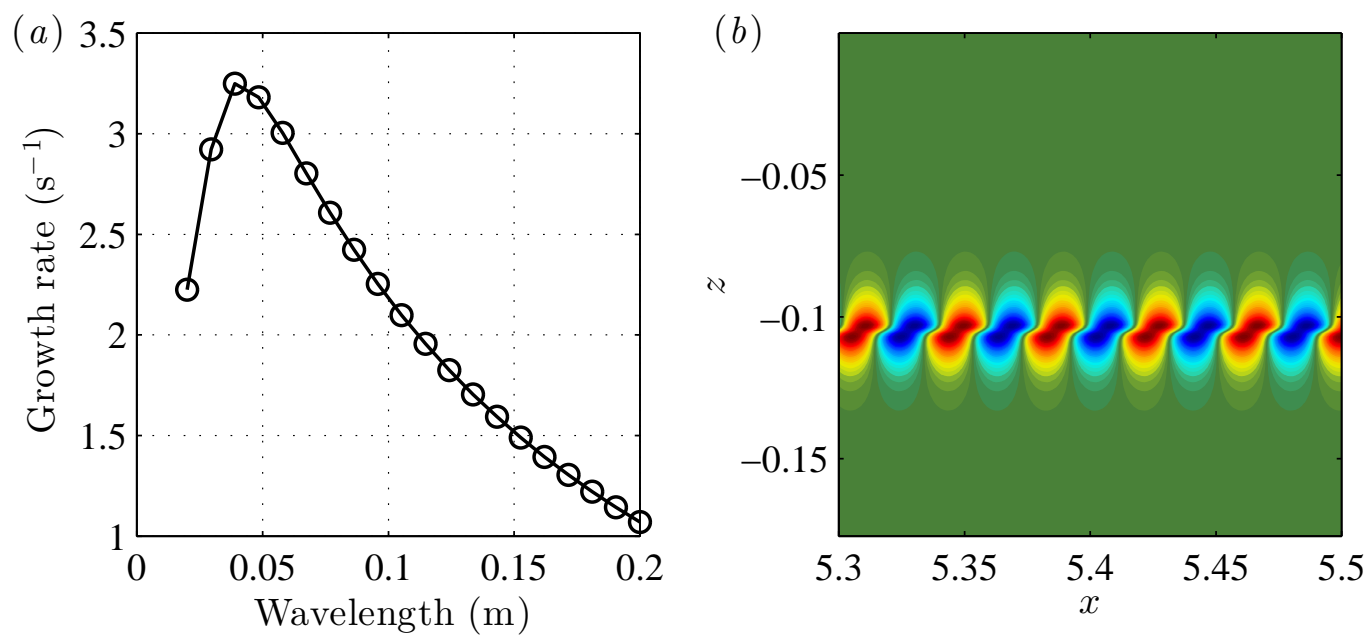

Figure 3.6: Analysis of shear instability along $x=5.38 \mathrm{~m}$ at $t=39 \mathrm{~s}$. Panel $(a)$ : growth rate of waves of disturbance. Panel $(b)$ : filled contour plot of the stream function showing the $2 \mathrm{D}$ structure of the fastest growing wave.

0.25. Applying the numerical method discussed in section 2.4, we find that the fastest growing wave of disturbance has a wavelength of approximately $\lambda=5 \mathrm{~cm}$, as shown in figure $3.5(\mathrm{c})$. Although figure 3.3 suggests that the actual wave length (i.e. the distance between the billows) is slightly larger, this is still a reasonably good estimation, especially when taking the nonlinear effect and the possibility of an instability that is growing both spatially and temporally into account. The propagation speed of the fastest growing wave, determined by $c_{r} / k$ where $c_{r}$ is the real part of the phase speed $c$, is $8.55 \times 10^{-5} \mathrm{~m} / \mathrm{s}$. This result is consistent with the observation in figure 3.3 , where the billows are essentially stationary as they grow in the inertial frame of reference. The 2D structure of the fastest growing wave is shown in the contour plot of the stream function in figure $3.5(d)$.

As a comparison, the vertical profile from a further upstream location $x=5.38 \mathrm{~m}$ at $t=39 \mathrm{~s}$ is also analyzed, and the results are given in figure 3.6. Panel $(a)$ shows that the wavelength of the fastest growing wave is still close to $5 \mathrm{~cm}$, though the maximum growth rate is larger than that shown in figure $3.5(c)$. This is because this location is closer to the wave front, where the pycnocline is thinner and the flow is more unstable due to shoaling. On the other hand, the 2D structure of the fastest growing wave shown in panel $(b)$ is almost identical to figure $3.5(d)$. In conclusion, the flow field satisfies all assumptions of the linear stability theory for a typical stratified shear flow as discussed in section 2.4, while the simulation results shown in figure 3.3 can be well explained by the solution of 
the Taylor-Goldstein equation. Hence, we conclude that it is the shear instability that is occurring at the edge of the recirculating core during the wave's shoaling. We should point out though, the reason why the linear stability analysis does not agree exactly with the simulation results but only provides an approximate solution is that the flow about the wave crest is not exactly parallel, and there exists the possibility of a spatial growing instability in the flow field.

\subsubsection{Bottom Shear Stress}

As mentioned earlier, in this case the configuration of the density profile results in a pycnocline that interacts with the bottom boundary. Consequently, the dense fluid is

(a)

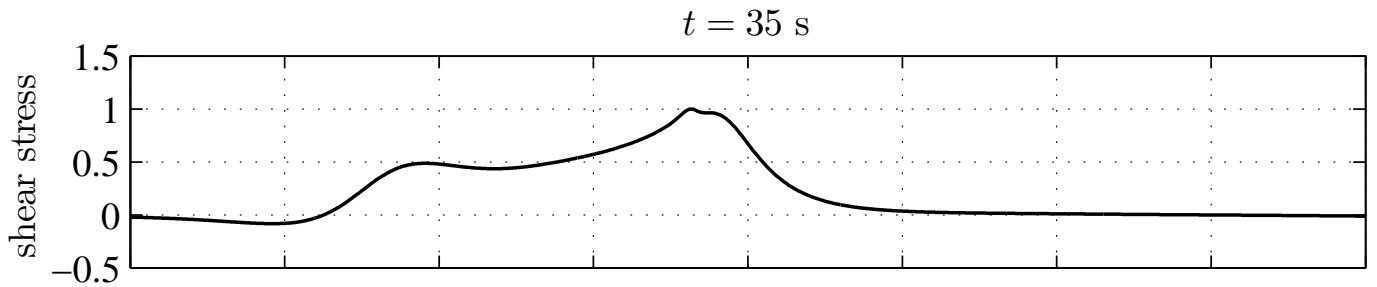

(b)

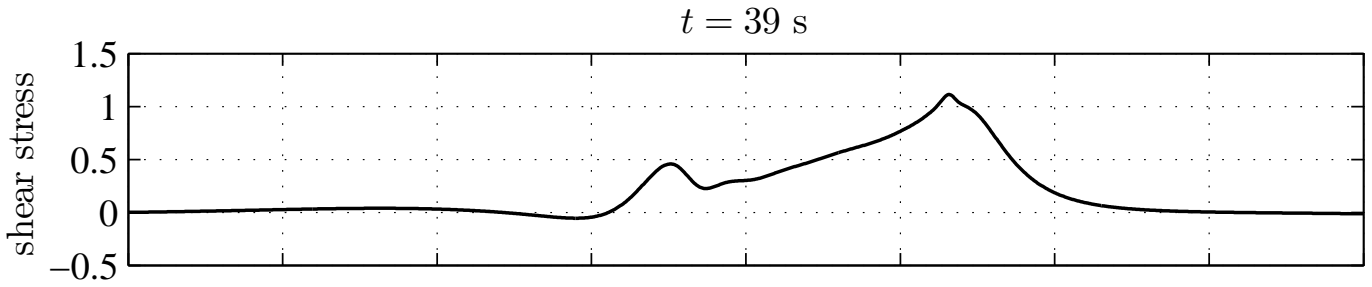

(c)

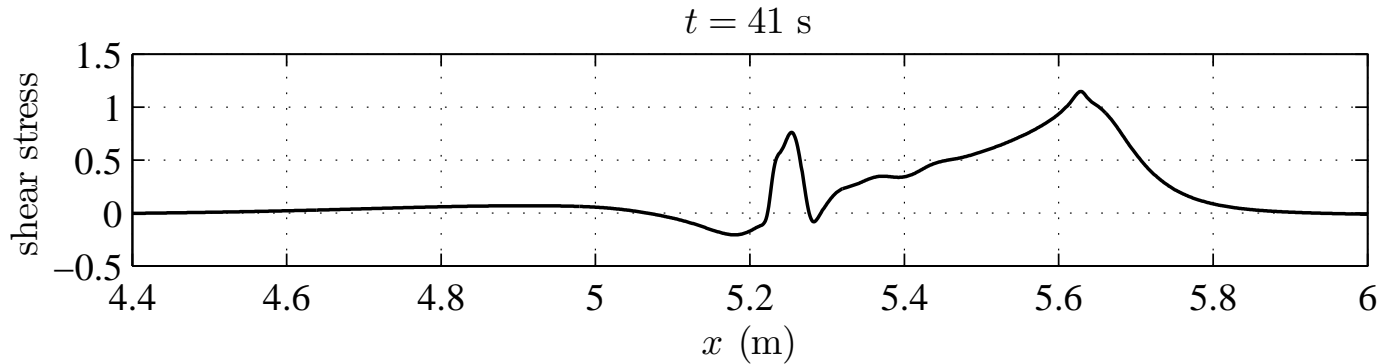

Figure 3.7: Evolution of the bottom shear stress during the wave passage. All subplots are normalized by the maximum shear stress found at $t=35 \mathrm{~s}(a)$. The average slope of the bottom boundary between $x=4.4 \mathrm{~m}$ and $6 \mathrm{~m}$ is approximately $0.75 \%$. 
absent on the top of the shelf, and the shoaling wave essentially propagates as a gravity current. Due to the no-slip boundary condition, the dense fluid in the core is then lifted up and over a small portion of light fluid that is trapped on the BBL, as shown in figure $3.4(b)$. This is the typical behavior of a gravity current head. As we shall see in the three-dimensional simulations in the next chapter, it leads to non-hydrostatic instability known as the lobe-cleft instability that grows rapidly in the spanwise direction. However, because the flow is restricted in two dimensions, the instability is not evident here.

Nevertheless, the interaction of the wave front with the BBL significantly increases the bottom shear stress, as shown in figure 3.7. With reference to the density contours in figures 3.2 and 3.3, we notice that the location of largest shear stress always corresponds to the foremost part of the wave. However, the solutions of the DJL equation suggests that ISWs are symmetric about their crests, which implies that the largest horizontal velocity, and hence the largest shear stress, would also occur at the wave crest. In fact, as shown in figure 3.2, the shift of the location at which the maximum bottom shear stress occurs is consistent with the loss of symmetry as the wave shoals. The change of bottom topography alters the wave such that it is no longer an exact solitary wave.

On the other hand, while the magnitude of the maximum shear stress at the wave front increases only slightly during the wave's shoaling, the secondary high shear region, located at the back of the wave where the billows interact with the BBL, develops rapidly over time. As shown in figure $3.7(c)$, the shear stress in this region eventually becomes comparable with the maximum shear stress. This is a clear indication that in addition to the shoaling behavior, the development of the Kelvin-Helmholtz billows also contributes to the bottom shear stress, which provides an additional possible mechanism for boundary layer mixing and sediment resuspension. 


\subsection{Medium and Higher Pycnoclines}

In this section we shall consider stratifications with higher pycnoclines. The two simulations to be discussed are labeled as Medium and Higher in table 3.1, whose pycnoclines are centered at $z_{0}=-0.16 \mathrm{~m}$ and $-0.15 \mathrm{~m}$, or $1.5 \mathrm{~cm}$ and $2.5 \mathrm{~cm}$ above the crest of the bottom topography, respectively. As a result, no gravity current is generated and the ISWs maintain their solitary wave-like form throughout the simulation.

We shall first discuss the Medium pycnocline case. The propagation of the wave is shown in figure 3.8. In contrast to the previous case where the instability is generated during the shoaling process, here the instability is more relevant behind the main wave after it passes the shelf. In the rest of this section, the breaking of the wave and the generation of the instability will be discussed in detail.
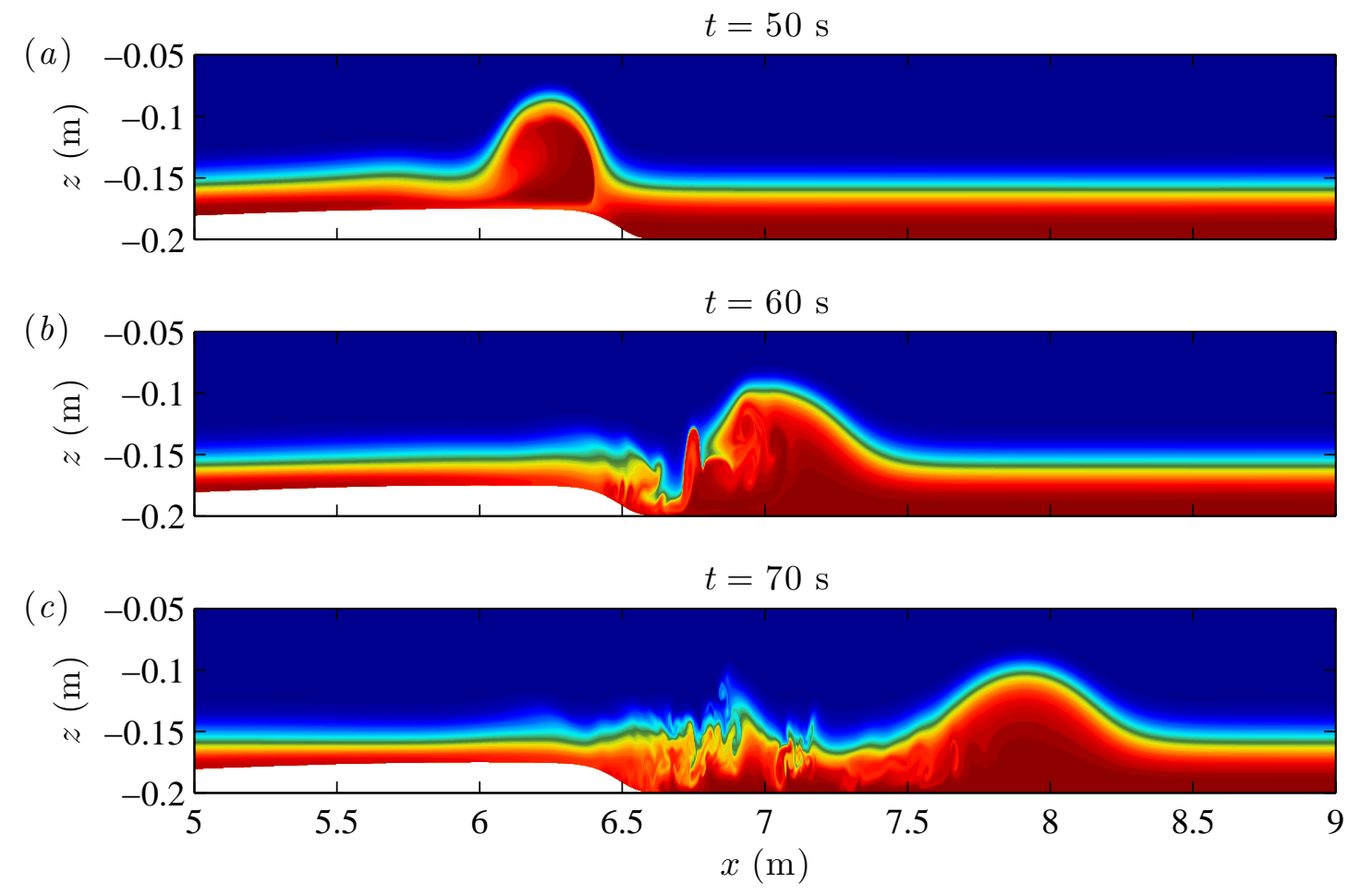

Figure 3.8: Shaded density contours of the simulation labeled as Medium showing the wave propagation at $(a) t=50 \mathrm{~s},(b) t=60 \mathrm{~s}$ and $(c) t=70 \mathrm{~s}$. Note that periodic boundary conditions are used in the $x$-direction with a period $L_{x}=7 \mathrm{~m}$. 


\subsubsection{Shoaling Behavior}

The early stage of the wave propagation (i.e. before $t=50 \mathrm{~s}$ ) will not be discussed here, since it follows an evolution similar to the Base Case, except that no shear instability is generated. At $t=50 \mathrm{~s}$, the wave has just passed over the crest of the bottom topography. As shown in figure $3.8(a)$, no evidence of shear instability has been found at the edge of the wave yet, though the core has already begun to overturn and break.

To explain the absence of shear instability, consider the Richardson number criterion given by equation (2.37). As shown in table 3.1, the difference between the maximum and minimum horizontal velocities of the initial wave is $12.28 \mathrm{~cm} / \mathrm{s}$ for the Medium pycnocline case, about $20 \%$ less than the difference of $15 \mathrm{~cm} / \mathrm{s}$ for the Base Case. Hence, the horizontal velocity gradient $u_{z}$ is also expected to be smaller during the wave's shoaling (here $u_{\max }=$ $10.07 \mathrm{~cm} / \mathrm{s}$ at $t=50 \mathrm{~s}$, whereas in the Base Case, $u_{\max }=10.16 \mathrm{~cm} / \mathrm{s}$ already at $t=35 \mathrm{~s}$ ). On the other hand, both cases have the same pycnocline width $d$ and density difference $\Delta \rho$, meaning that their buoyancy frequency profiles across the shear layer are also identical. The local Richardson number across the wave crest at $t=50 \mathrm{~s}$ is approximately 0.31 , much larger than that in the Base Case (which is about 0.065). Hence, during the shoaling process the flow is not able to generate enough shear stress to initiate any instability.

In figure $3.8(a)$, we also notice that behind the main wave there is a secondary peak of the pycnocline, located at $x \approx 5.7 \mathrm{~m}$. The corresponding vertical velocity plot is given in figure 3.9, which suggests that the flow pattern in this region is typical of an ISW, despite the fact that it is much weaker than the main wave (the vertical velocity is approximately $1 / 30$ in absolute value of the maximum/minimum vertical velocity of the main wave). This implies that a secondary ISW is being fissioned behind the main wave during the shoaling process.

An explanation of this phenomenon would be that the rise of bottom boundary essentially shifts the pycnocline away from mid-depth, such that the main wave has to change its shape in order to adjust to the new environment. Since the shoaling wave is narrower than the initial wave, the extra dense fluid is left behind, from which a second wave is generated. This hypothesis can be justified by comparing the simulation result with the solution of the DJL equation. Such comparison is given in figure 3.10, in which the DJL solution is obtained in a channel with a depth of $-0.175 \mathrm{~m}$ and the same stratification as the simulation, but less available potential energy because of the shoaling process. The resulting density profiles suggest that the DJL solution fits the simulation result almost exactly, meaning that on the top of the shelf, the shoaling wave still behaves like a solitary wave, and analyses of ISWs (instead of gravity currents) are applicable. 


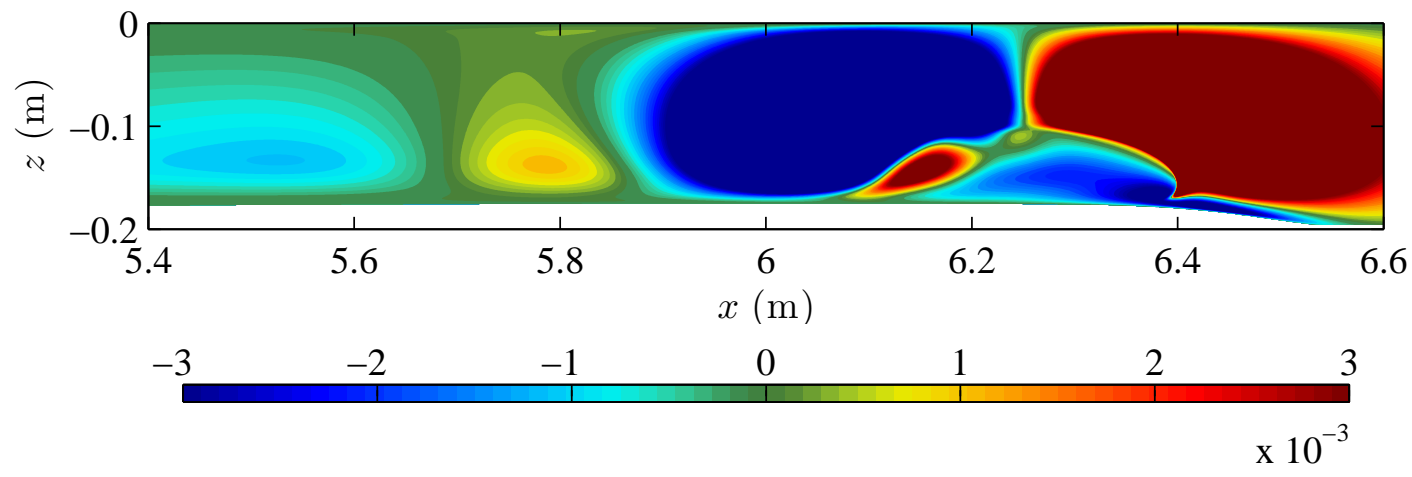

Figure 3.9: Vertical velocity plot at $t=50 \mathrm{~s}$. The color axis is saturated at $w= \pm 0.3 \mathrm{~cm} / \mathrm{s}$, with positive (upward) velocity shown in red and negative (downward) velocity shown in blue. Note that the maximum and minimum vertical velocities at $t=50 \mathrm{~s}$ are $w_{\max }=3.84$ $\mathrm{cm} / \mathrm{s}$ and $w_{\min }=-3.37 \mathrm{~cm} / \mathrm{s}$, respectively.
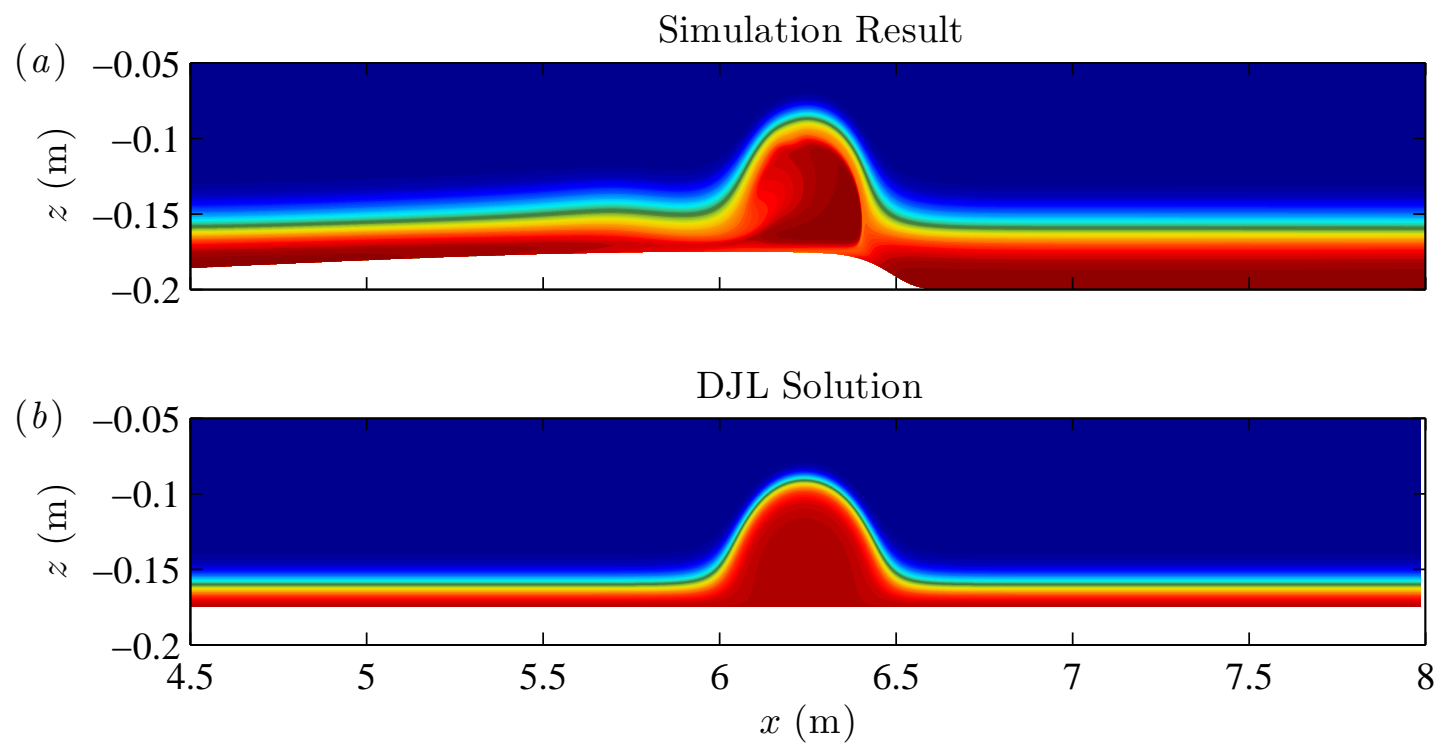

Figure 3.10: Density contour plots of the wave at $t=50 \mathrm{~s}$ obtained from $(a)$ the simulation result and $(b)$ the solution of the DJL equation with the bottom boundary located at $z=-0.175 \mathrm{~m}$, the crest of the bottom topography in the simulation domain. 


\subsubsection{Wave Breaking and the Vortex-Rich Region}

More interesting dynamics of the flow occurs after the wave propagates down the shelf. As shown in panels $(b)$ and $(c)$ of figure 3.8, after the de-shoaling process the core is completely broken up and washed out of the main wave, yielding a vortex-rich region of fluid ejected from the core. In the mean time, the remaining fluid forms a new solitary wave in front of the broken core, which propagates just like the original wave.

One of the consequences of the wave's de-shoaling process is that, as the core breaks up, a portion of the mass and energy carried by the original wave is trapped in this vortex-rich region, which does not propagates with the wave. As a result, the newly generated wave has less available potential energy. A comparison between the new wave at $t=80 \mathrm{~s}$ and the initial wave is given in figure 3.11. The most notable difference is that the new wave appears smaller (or narrower) than the initial wave. Also, due to diffusion the pycnocline is wider at $t=80 \mathrm{~s}$. In terms of other features, however, the new wave behaves very similar to the original wave. Some relevant wave parameters obtained at $t=80 \mathrm{~s}$ are $u_{\max }=6.72$ $\mathrm{cm} / \mathrm{s}, u_{\min }=-5.22 \mathrm{~cm} / \mathrm{s}$ and $w_{\max }=1.73 \mathrm{~cm} / \mathrm{s}$, all of which are very close to those for the initial wave shown in table 3.1 .
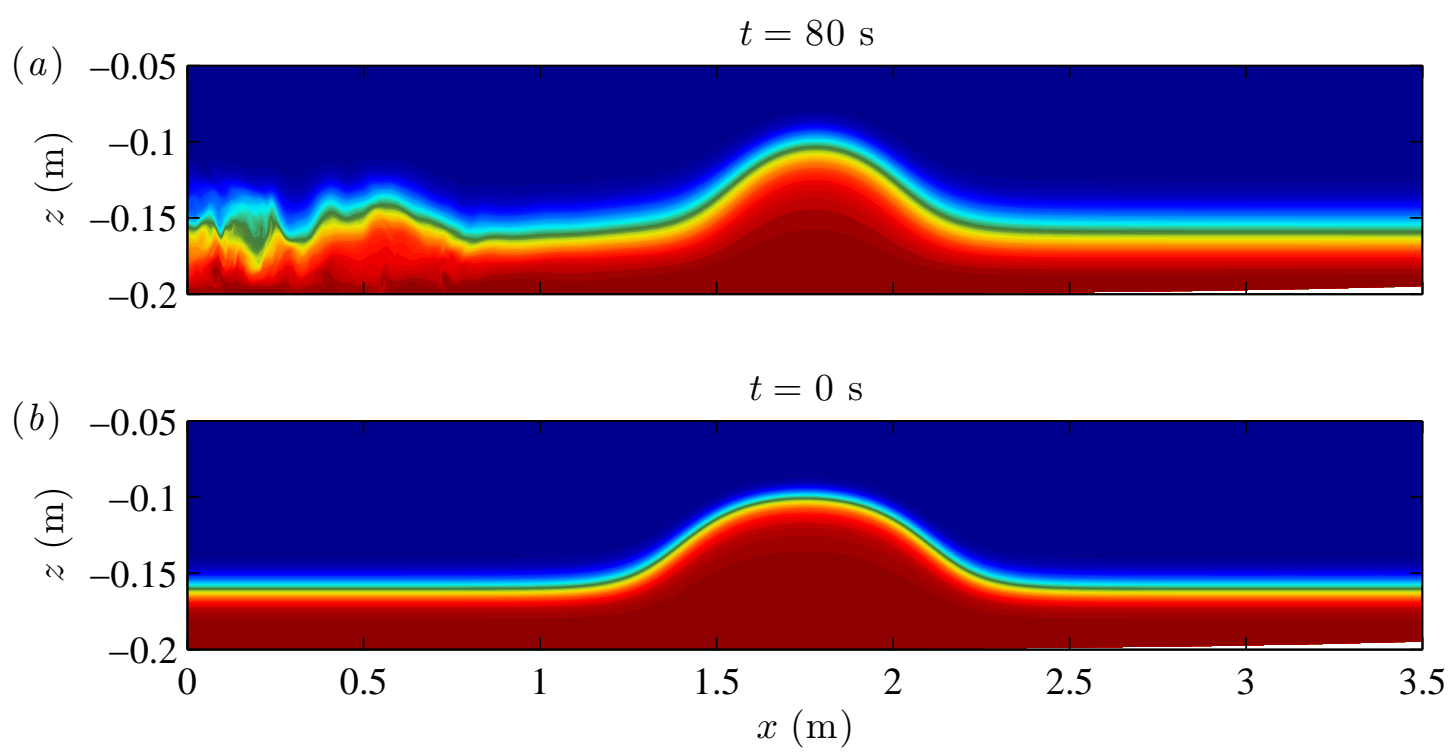

Figure 3.11: Comparison of the density profiles of $(a)$ the newly generated wave at $t=80$ $\mathrm{s}$ and $(b)$ the initial wave at $t=0 \mathrm{~s}$. Because of the periodic boundary condition, these waves are essentially at the same location. 
The evolution of the vorticity field showing the wave's de-shoaling process and the formation of the vortex-rich region is given in figure 3.12. Recall from equation (2.8) that in a 2D flow field, the rate of change of vorticity is determined by the horizontal density gradient (baroclinic vorticity) and the viscosity (diffusion of vorticity). In this figure, the baroclinic vorticity (with negative sign) is found along the high shear region at the core's edge and the wave crest, whereas the vorticity production due to viscosity (with positive sign) occurs along the BBL and corresponds to the increasing bottom shear stress during the wave passage.

Figure 3.12 also suggests that the formation of the vortex-rich region is associated with the breaking up of the core, which takes place as soon as the main wave propagates down the shelf. As the core breaks up, mixing of the dense and light fluid occurs across the deformed pycnocline at the back of the wave, such that the baroclinic vorticity is also found inside the broken core. At the same time, the positive vorticity from the viscous boundary layer enters into the core due to boundary layer separation, interacting with the baroclinic vorticity and creating the vortex-rich region behind the wave. Figures $3.12(d)$ and $3.13(a)$ show that, at $t=60 \mathrm{~s}$, these two types of vorticity interact with each other at the edge of the vortex between $x=6.7 \mathrm{~m}$ and $6.8 \mathrm{~m}$, along the deformed pycnocline in the vortex-rich region. The interaction increases the magnitude of both positive and negative vorticity significantly $\left(\omega_{\max } \approx 22.36 \mathrm{~s}^{-1}\right.$ and $\left.\omega_{\min } \approx-25.74 \mathrm{~s}^{-1}\right)$, such that the color map is over saturated in this area.

The boundary layer separation occurs in the down-slop portion of the shelf, as shown in the detailed plots in figure 3.13. While positive vorticity is expected in the viscous boundary layer during the wave passage, panel $(a)$ shows that extremely strong negative vorticity (with a magnitude of $\omega_{\min } \approx-109.7 \mathrm{~s}^{-1}$ ) is found along the bottom boundary between $x=6.5 \mathrm{~m}$ to $6.7 \mathrm{~m}$. The negative vorticity is induced by the negative horizontal velocity found in this area, as suggested in the horizontal velocity plot in panel $(b)$. It indicates that the flow has reversed its direction in the boundary layer and flow separation has occurred. This downstream flow increases the bottom shear stress significantly. In panel $(c)$, extremely strong negative shear stress is found near $x=6.7 \mathrm{~m}$. The absolute value of the shear stress in this point has a magnitude of as much as 3 times that of the positive stress induced by the wave passage. The separation point, defined as the point between the forward and backward flow, is found near $x=6.4 \mathrm{~m}$ (recall that the crest of the bottom topography is located at $x=6 \mathrm{~m}$ ), where the bottom shear stress is zero. As the flow separation grows, vortices are generated along the separation streamline (shown in figure $3.12(d))$. The vortices then break down into smaller scale instability, and the mechanical energy carried by these vortices is eventually converted into internal (heat) energy as the mixing process further breaks the instability down into molecular scale. 

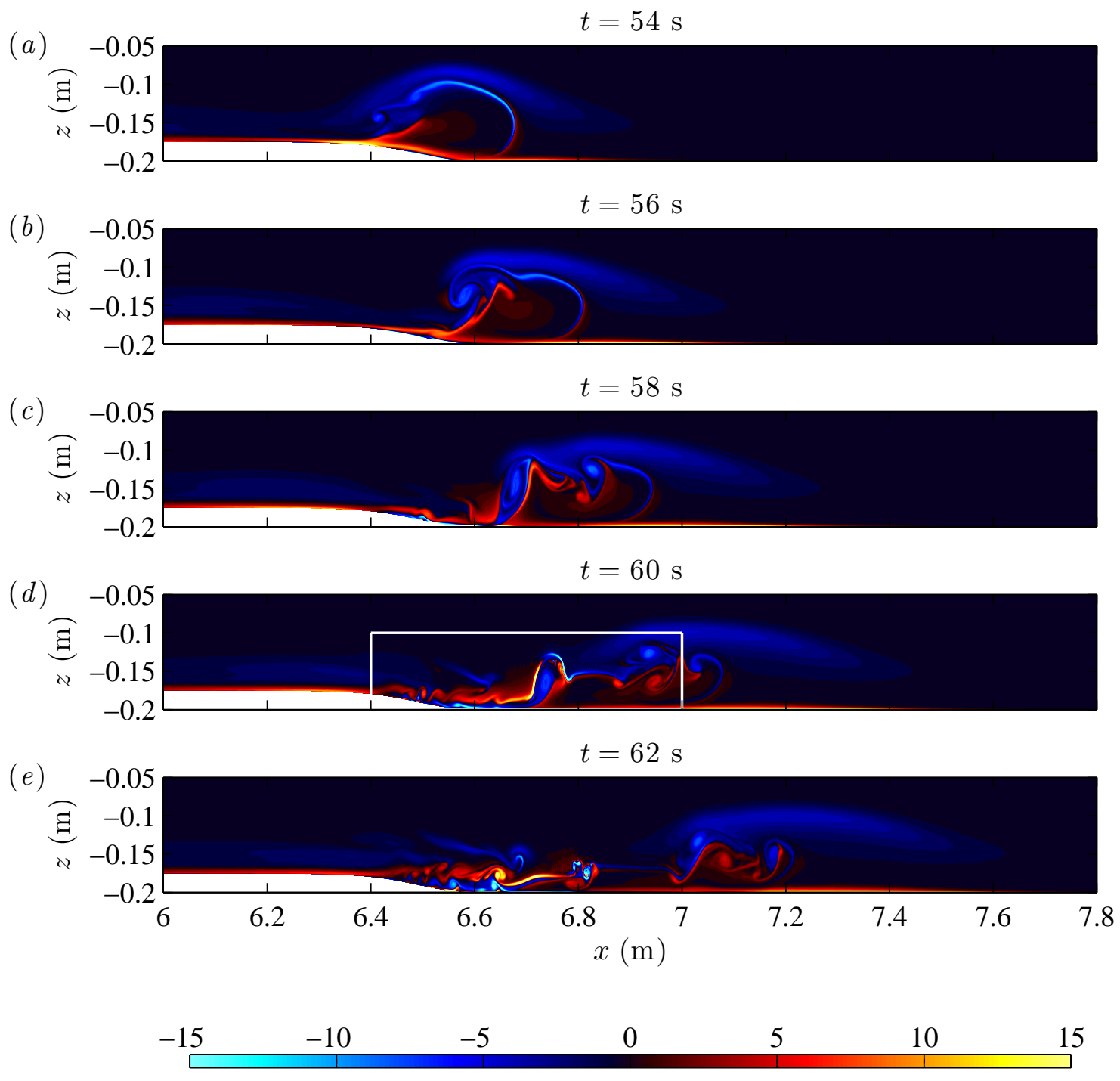

Figure 3.12: Vorticity field plots from $t=54 \mathrm{~s}$ to $62 \mathrm{~s}$. The color axis is saturated at $\omega= \pm 15 \mathrm{~s}^{-1}$, with positive vorticity shown in red (hot), negative vorticity shown in blue (cold) and irrotational flow shown in black. The pycnocline is not shown here because the plots will otherwise be too busy. Nevertheless, location of the wave crest can be visualized by the negative baroclinic vorticity at $z \approx-0.1 \mathrm{~m}$. Detail of the vortex-rich region as indicated by the white box in panel $(d)$ is given in figure 3.13. 
$(a)$

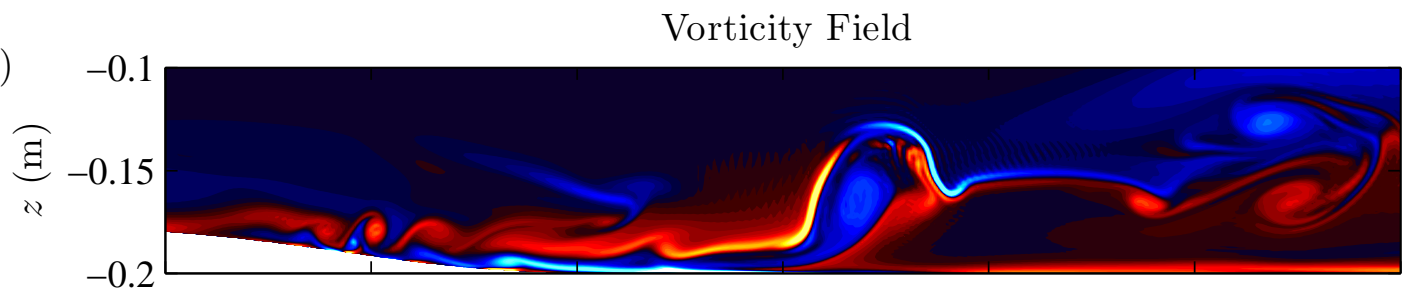

(b)

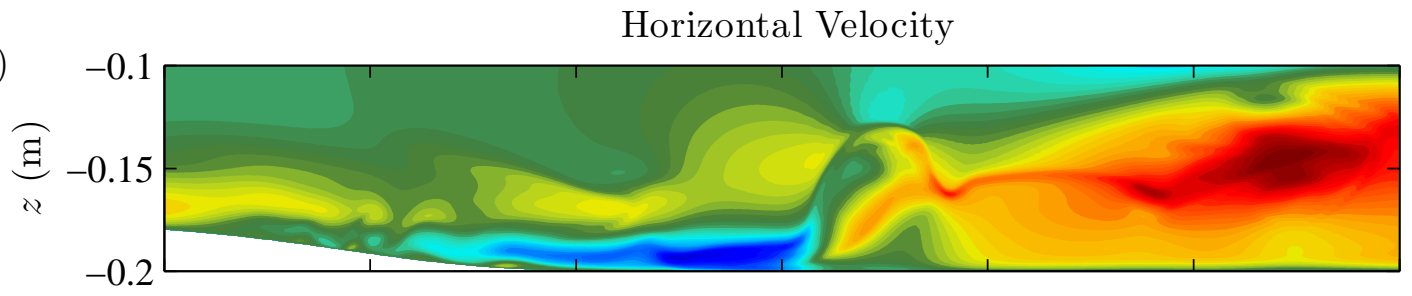

(c)

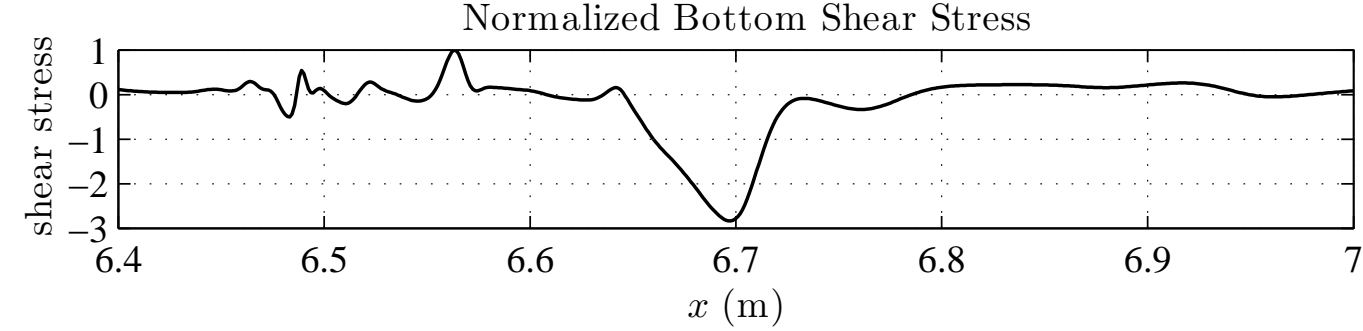

Figure 3.13: Detail of the vortex-rich region and the bottom boundary layer at $t=60 \mathrm{~s}$ given by $(a)$ vorticity field, $(b)$ horizontal velocity and $(c)$ bottom shear stress plots. For panel $(a)$ the color axis is the same as figure 3.12, whereas for panel $(b)$ it is saturated at $\pm 0.1 \mathrm{~m} / \mathrm{s}$ with positive velocity shown in red, negative velocity shown in blue and zero velocity shown in green. Panel $(c)$ is normalized by the maximum positive bottom shear stress found in the plot.

The combined effect of core breaking and boundary layer separation makes the vortexrich region a hot spot for material mixing, where sediment resuspension is very likely to occur. In fact, the instability in this region is expected to develop into a completely threedimensional state. For this reason, three-dimensional simulation of this case has been performed and the results will be discussed in section 4.2. Additionally, although no shear instability is produced along the core's edge because the shear stress is not strong enough, in the three-dimensional simulation the lobe-cleft instability is observed during the shoaling process, despite the fact that the shoaling wave is better described as a solitary wave rather than a gravity current. Nevertheless, the 2D simulation has provided a reasonably realistic result in terms of the overall flow structure because the mean flow is two-dimensional. 


\subsubsection{The Higher Pycnocline Case}

To close this section, let us consider the Higher pycnocline case. The wave's de-shaoling process from $t=50 \mathrm{~s}$ to $90 \mathrm{~s}$ is shown in figure 3.14 with three panels of density contours. Panel $(a)$ suggests that, when the wave is on the top of the shelf, it behaves very similar to the wave in the Medium pycnocline case (see figure 3.8 ( $a$ ) for a comparison). No shear instability is generated in this case either, because the higher pycnocline makes the horizontal velocity gradient across the shear layer even smaller than the other cases (at $t=50 \mathrm{~s}, u_{\max }=8.73 \mathrm{~cm} / \mathrm{s}$ and $\left.u_{\min }=-6.67 \mathrm{~cm} / \mathrm{s}\right)$. With the same density gradient, the shear stress is also smaller. Additionally, for exactly the same reason, the wave becomes narrower during the shoaling process in order to adjust to the elevated bottom boundary, and a secondary ISW is fissioned behind it.

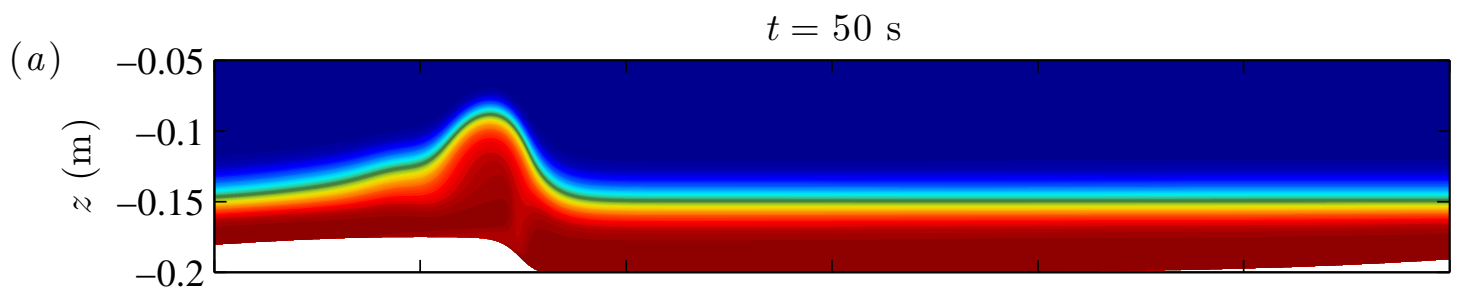

$t=70 \mathrm{~s}$
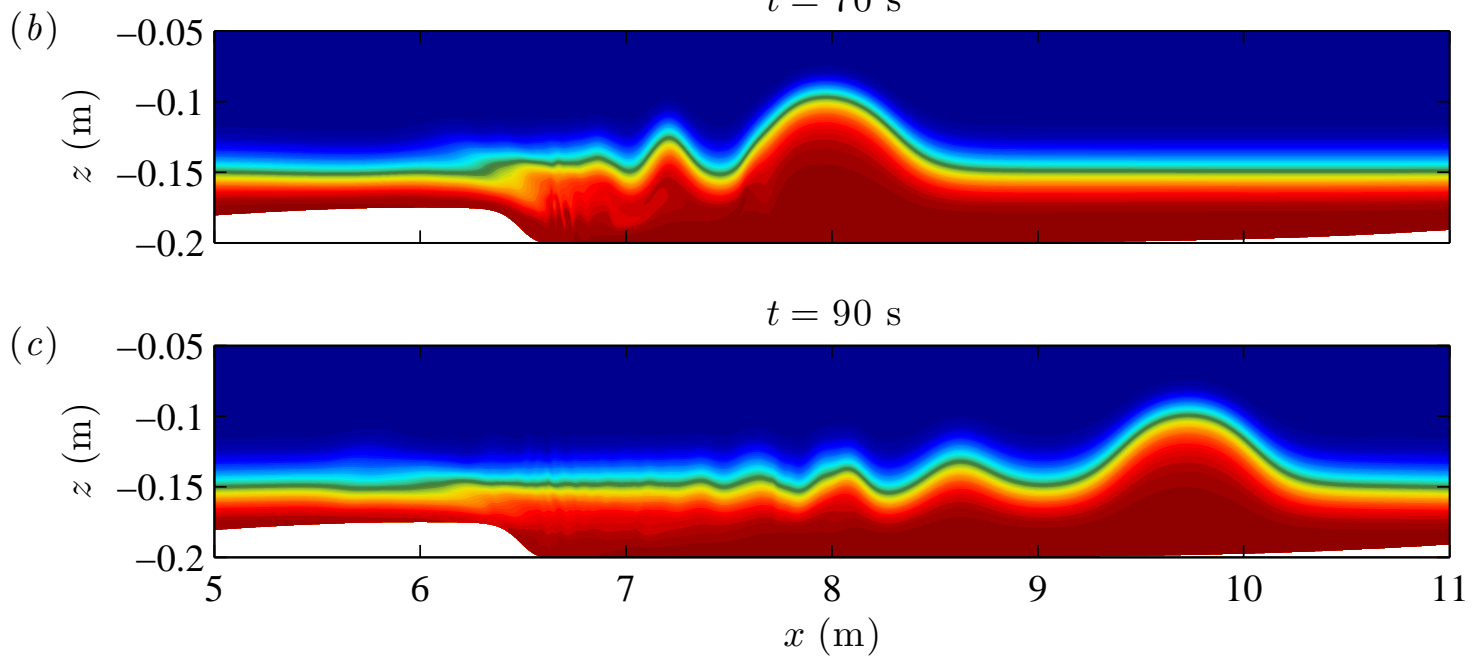

Figure 3.14: Density contours showing the de-shoaling of the wave and the formation of the wave train in the Higher pycnocline case at $(a) t=50 \mathrm{~s},(b) t=70 \mathrm{~s}$ and $(c) t=90 \mathrm{~s}$. 
The flow structure becomes different from the Medium pycnocline case after the wave passes the top of the bottom shelf. Panels $(b)$ and $(c)$ suggest that, while the break up of the core still occurs as the main wave propagates down the shelf, the vortex-rich region is not observed. Instead, as the core moves out of the main wave, the dense fluid carried by the core forms the narrow wave behind the main wave seen in panel $(b)$, which further fissions into several even smaller amplitude, broader ISWs shown in panel $(c)$. The excess energy is, in part, carried away by the propagation of these waves. Due to loss of energy, subsequently generated waves have a smaller amplitude and propagate at a slower speed than their predecessors (as shown in the figure, distances between adjacent waves at $t=90 \mathrm{~s}$ are larger than those at $t=70 \mathrm{~s}$ ). 


\subsection{A Corrugated Bottom Boundary}

Unlike the laboratory environment, in oceans and lakes the bottom topography is expected to be non-uniform. Because the trapped core of ISWs of elevation interacts directly with the BBL during the shoaling process, any perturbation to the bottom topography will necessarily alter the nature of the flow. A combined experimental and numerical study of ISWs propagating over a corrugated bottom boundary has been reported in Carr et al. (2010). In the case of waves of elevation, it is found that two different mechanisms are responsible for the generation of turbulent mixing in the water column: the boundary layer separation at the crest of each individual corrugation caused by the ISW-induced current, from which lee vortices are subsequently formed, and the shear instability in the upstream portion of the wave, which occurs when the bottom corrugations reach into (or very close to) the pycnocline at rest.

The present work does not attempt to reproduce the results presented in the previous literature. The purpose of this investigation is to assess the influence of bottom corrugations on the behavior of shoaling wave. Since this is a short introductory section, variation of amplitude and wavelength of the corrugations will not be considered, and attention is restricted to short length scale undulations of the form

$$
\epsilon(x)=0.1 \sin (20 \pi x),
$$

which have a wavelength $\lambda=0.1 \mathrm{~m}$ and an amplitude equivalent to $20 \%$ of the local height of the bottom shelf (the definition equations of the bottom topography is given in equations (3.1)). As a reference, the largest corrugation (which is on the crest of the bottom topography) has an amplitude of $0.5 \mathrm{~cm}$, which is $2.5 \%$ of the total depth or $5 \%$ of the wavelength (peak-to-peak distance). Since the horizontal grid spacing is approximately 1 $\mathrm{mm}$ and the vertical grid points are naturally clustered near the bottom boundary because of the Chebyshev discretization, the flow within each corrugation is well resolved.

The simulation presented in this section is labeled as Rough in table 3.1, which has the same density profile as the Medium pycnocline case. Since in both cases the overall flow structure is similar except for the flow in the BBL, the propagation of the wave is not shown here. Instead, detailed plots of the wave and the BBL at $t=50 \mathrm{~s}$ are given in figures 3.15 and 3.16. As expected, the vorticity plot in figure 3.15 shows that baroclinic vorticity is found along the wave crest and the core's edge, while vorticity production due to viscosity is found in the BBL. However, the baroclinic vorticity suggests that the Kelvin-Helmholtz billows are starting to form along the core's edge, indicating that the shear stress is strong enough for the shear instability to be generated in this case. Since this feature is absent 


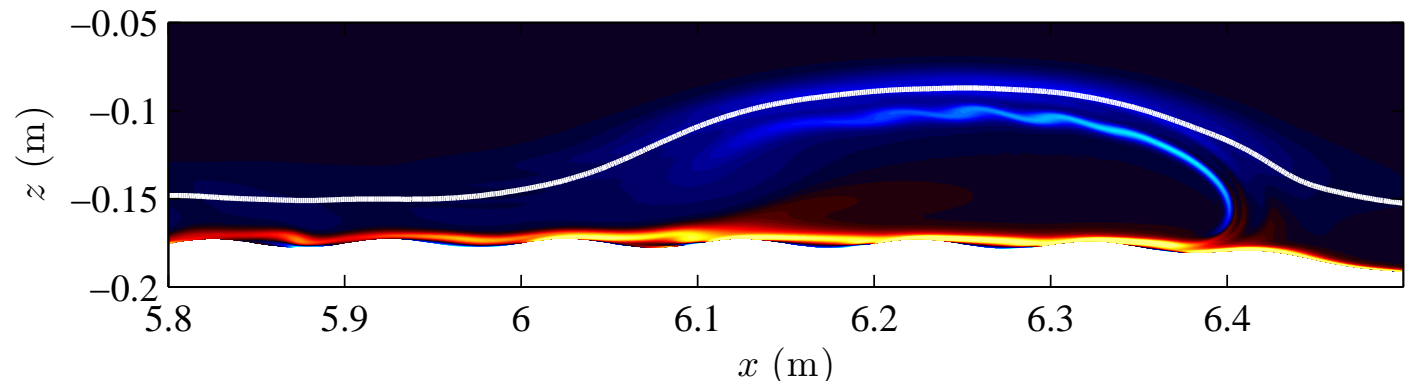

Figure 3.15: Vorticity field of the Rough bottom boundary case at $t=50 \mathrm{~s}$. The the color axis is the same as in figure 3.12. The solid curve denotes the location of the pycnocline. Detail of the flow in the boundary layer is given in figure 3.16.

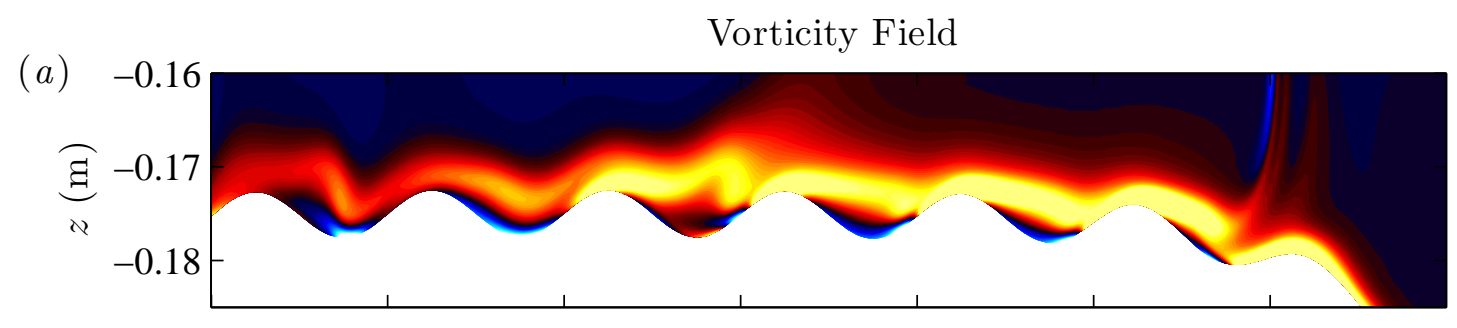

Horizontal Velocity

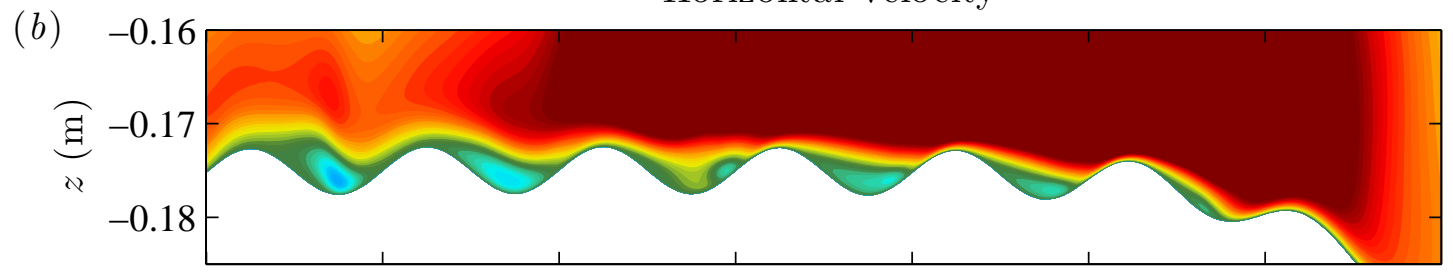

(c)

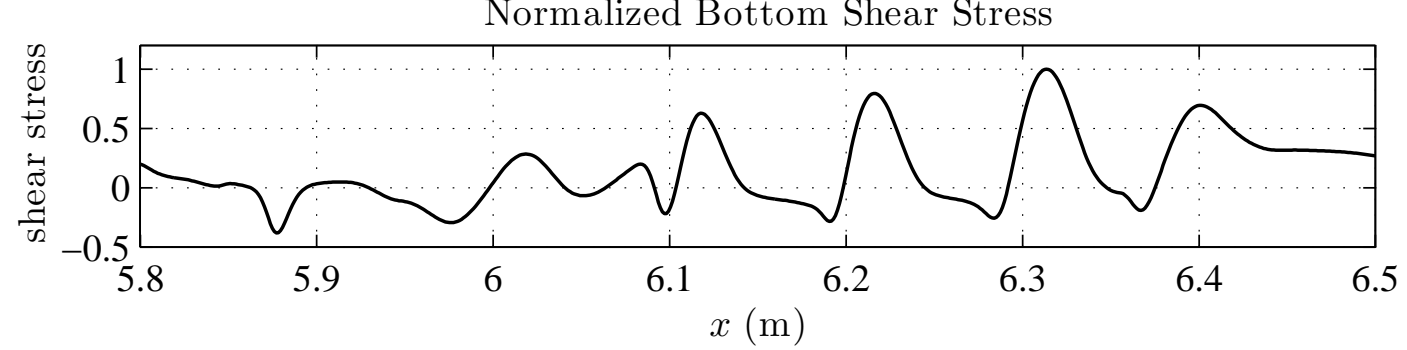

Figure 3.16: Detail of the bottom boundary layer at $t=50 \mathrm{~s}$ given by $(a)$ vorticity field, (b) horizontal velocity and $(c)$ bottom shear stress plots. For panel $(a)$ the color axis is the same as figure 3.12 , whereas for panel $(b)$ it is saturated at $\pm 0.04 \mathrm{~m} / \mathrm{s}$ with positive velocity shown in red, negative velocity shown in blue and zero velocity shown in green. Panel $(c)$ is normalized by the maximum positive bottom shear stress found in the plot. 
in the Medium pycnocline case, we conclude that the existence of bottom corrugations contributes to the shear stress in the upstream portion of the wave and the leading edge of the core where the billow formation is originated.

On the other hand, figure 3.16 shows that boundary layer separation has taken place inside the bottom corrugations. In panel $(a)$, negative vorticity is found at the trough of each corrugation above the bottom boundary. The region of negative vorticity in the corrugation 'valleys' corresponds to the region below the separation streamline, where negative horizontal velocity is found as shown in panel $(b)$. The corresponding bottom shear stress profile is plotted in panel $(c)$. In agreement with the previous results, the bottom shear stress is dominated by oscillations with a wavelength that corresponds to that of the bottom corrugations. More specifically, the upstream-directed (positive) shear stress always occurs at the crest of each corrugation and corresponds to the positive vorticity, whereas downstream-directed (negative) stress occurs at the trough of each corrugation and corresponds to the negative vorticity.

Unlike in the experiments and simulations performed by Carr et al. (2010) where vortices are subsequently formed from these corrugations, in our simulation the flow inside these 'valleys' is not energetic enough to self advect away from the boundary layer. In fact, as shown in figure 3.16, the magnitudes of the negative vorticity, horizontal velocity and bottom shear stress induced by the downstream-directed flow in the trough of the corrugations are only a fraction of those found in the main flow above the boundary layer. The amount of energy carried by the downstream-directed flow inside the bottom corrugations is determined by the size and shape of these corrugations, as well as the stratification of the fluid. A deeper and larger corrugation is expected to have the ability to trap more energy from the main flow, such that the backward flow inside the boundary layer is stronger and the the boundary layer separation grows rapidly. An example is the de-shoaling process of the wave discussed in the Medium pycnocline case, in which a vortex-rich region is formed in the down-slop portion, or the 'valley', of the shelf. Disturbance to the pycnocline behind the wave may also be more significant if the pycnocline is thicker, because with a thicker pycnocline there is the opportunity to excite more modes.

We shall mention that while this simulation is performed in the laboratory scale, its results have many implications for larger scales. Because in the field scale the Reynolds number is much larger, the flow is expected to be more turbulent. As a result, the viscous effect is smaller and the boundary layer is thinner. Hence, disturbance to the flow due to the rough bottom boundary could be stronger. For example, the lee vortices which are absent in the present simulation may appear in field scale simulations. In addition, Carr et al. (2010) also suggest that variation of wavelength and amplitude of the bottom corrugations will affect the resulting turbulent mixing, because these properties are directly 
related to the amount of energy carried by the flow inside these corrugations. Therefore, to further understand this topic, the next step is to experiment with field scale flows and various sizes and shapes of bottom corrugations. 


\section{Chapter 4}

\section{Three-Dimensional Simulations}

Due to the limitation of available computational resources, 2D simulations are much easier to carry out and analyze compared with their three-dimensional (henceforth 3D) counterparts. However, in the real world, 2D flows only describe a small subset of environmental and geophysical fluid flows. The most directly observable difference is that, because of the absence of spanwise velocity, vortex stretching and tilting does not occur in two dimensions. Recall from section 2.1 that the vorticity equation is given by

$$
\frac{D \boldsymbol{\omega}}{D t}=(\boldsymbol{\omega} \cdot \nabla) \boldsymbol{u}+\nu \nabla^{2} \boldsymbol{\omega}+\nabla \times \rho \boldsymbol{g},
$$

where $(\boldsymbol{\omega} \cdot \nabla) \boldsymbol{u}$ represents the rate of change of vorticity due to the stretching and tilting of vortex lines. In two dimensions, equation (4.1) reduces to a single scalar equation

$$
\omega_{t}+u \omega_{x}+w \omega_{z}=\nu\left(\omega_{x x}+\omega_{z z}\right)+\rho_{x} g,
$$

in which the term $(\boldsymbol{\omega} \cdot \nabla) \boldsymbol{u}$ is absent. Since a complete vorticity field is required to properly describe the development of turbulence, while nearly all macroscopic flows associated with instabilities are turbulent, 3D dynamics are required to fully understand the generation and evolution of these instabilities.

Taking advantage of modern, high-performance computers and computer clusters ${ }^{1}$ (Loken et al., 2010), 3D simulations of the Base Case and the Medium pycnocline case have

\footnotetext{
${ }^{1}$ Computations were performed on the gpc supercomputer at the SciNet HPC Consortium. SciNet is funded by: the Canada Foundation for Innovation under the auspices of Compute Canada; the Government of Ontario; Ontario Research Fund - Research Excellence; and the University of Toronto
} 


\begin{tabular}{lccccl}
\hline Simulation & $\begin{array}{c}\text { Initial time } \\
t_{0}(\mathrm{~s})\end{array}$ & $\begin{array}{c}\text { Width } \\
L_{y}(\mathrm{~m})\end{array}$ & $\begin{array}{c}\text { Grid size } \\
N_{y}\end{array}$ & $\begin{array}{c}\text { Diffusivity } \\
\kappa\left(\mathrm{m}^{2} / \mathrm{s}\right)\end{array}$ & Result \\
\hline Case 1 & 41 & 0.05 & 48 & $10^{-6}$ & Not physical \\
Case 2 & 35 & 0.05 & 48 & $10^{-6}$ & Gibbs phenomenon \\
Case 3 & 35 & 0.02 & 48 & $5 \times 10^{-6}$ & Billows remain 2D \\
Case 4 & 35 & 0.1 & 96 & $5 \times 10^{-6}$ & Discussed in section 4.1 \\
Medium & 40 & 0.05 & 48 & $5 \times 10^{-6}$ & Discussed in section 4.2 \\
\hline
\end{tabular}

Table 4.1: List of numerical parameters and summary of simulation results of 3D simulations. Cases 1-4 are initialized from the 2D Base Case, and Medium is initialized from the 2D Medium pycnocline case. In summary, in the Base Case the Gibbs phenomenon is observed in the cases with larger diffusivity, the generation of lobe-cleft instability occurs before $t=41$ s so that Case 1 is physically irrelevant, and the three-dimensionalizaiton of the Kelvin-Helmholtz billows is absent with small domain width.

been performed, and the results will be discussed in this chapter. The 2D simulations suggest that, since no instability is generated before the wave starts to shoal, the transition of the flows to a 3D state is not likely to occur prior to shoaling. Hence, for computational efficiency, all 3D simulations are initialized at some time $t_{0}>0$ from the 2D flow fields. The three-dimensionalization is seeded by a velocity perturbation of $0.1 \%$ in the form of multiplicative white noise applied to the $2 \mathrm{D}$ flow fields. The perturbation has no preferred spatial structure. If instabilities develop somewhat later in time, then the breakdown of the flows into a fully 3D state will occur naturally.

Since the primary flow is two-dimensional, variation of bottom topography in the spanwise direction is not considered in this work (in fact, the invariance of topography in the spanwise direction is one of the limitations of SPINS, see section 2.3). Several test runs with various initial time $t_{0}$, domain width $L_{y}$, spanwise resolution $N_{y}$ and diffusivity $\kappa$ have been performed of the Base Case. The results (summarized in table 4.1) suggest that 3D flows are more turbulent than their 2D counterparts in general, because the growth of instabilities is not restricted in a specific direction. As a result, properly resolving all small-scale features in the flow field becomes more challenging. For this reason, the simulations to be discussed in the following sections have a diffusivity $\kappa=5 \times 10^{-6} \mathrm{~m}^{2} / \mathrm{s}$ instead of $10^{-6} \mathrm{~m}^{2} / \mathrm{s}$, since it is not practical to further increase the resolution. The higher diffusivity essentially acts as a filter that smooths out any possible Gibbs phenomenon in the simulations. With the viscosity fixed at $\nu=10^{-6} \mathrm{~m}^{2} / \mathrm{s}$, the resulting Schmidt number is $S c=0.2$. Although $S c=1$ is commonly used in the literature of direct numerical 
simulations, considering the fact that the physical Schmidt number of water is around 500 this does not void the validity of our simulations.

For all 3D simulations, Fourier expansion with periodic boundary conditions is applied in the spanwise $(y)$ direction. Results of the test runs also suggest that the generation of lobe-cleft instability occurs with any domain width, whereas the three-dimensionalization of the Kelvin-Helmholtz billows depends on the domain width such that the 3D structure of the billows is only observed in Case 4. In this case, the width of the domain is set to be $L_{y}=0.1 \mathrm{~m}$ and is discretized into 96 equally spaced grid point. This gives a grid spacing of approximately $1 \mathrm{~mm}$, which is the same as in the $x$-direction. Since this simulation is most informative while it is well resolved, the first section of this chapter will focus on this case only. In particular, the emphasis is placed on the generation of the lobe-cleft instability and the three-dimensionalization of the Kelvin-Helmholtz billows, as well as the interaction of these instabilities with the bottom boundary layer (BBL).

On the other hand, since the shear instability is less relevant in the Medium pycnocline case, the domain width is set to be $L_{y}=0.05 \mathrm{~m}$ and the grid size is chosen to be $N_{y}=48$. Given the fact that the development of instability is slower and the simulation is more time consuming, a reduced domain width provides some computational efficiency in this case. The simulation results are discussed in section 4.2, focusing on the evolution of the vortexrich region of fluid ejected from the core after the shoaling and during the de-shoaling process. 


\subsection{The Base Case}

Before presenting results of the 3D simulation, let us quickly review some key features of the Base Case. In this case, the pycnocline is centered at $z_{0}=-0.1725 \mathrm{~m}$ with a thickness $d=0.02 \mathrm{~m}$. As a result, the shelf on the bottom boundary (whose crest is located at $(x, z)=(6,-0.175) \mathrm{m})$ reaches into the pycnocline, such that the shoaling wave essentially behaves like a gravity current on the top of the shelf. The $2 \mathrm{D}$ flow is characterized by the shear instability generated at the edge of the core, and the Kelvin-Helmholtz billows found in this high shear region. In this section, we will first analysis the 3D structure of the flow in detail, and then discuss the dynamics of the overall shoaling process, including the evolution of boundary layer instabilities.

\subsubsection{Three-Dimensional Flow Structure}

The overall 3D structure of the shoaling wave at $t=42 \mathrm{~s}$ is plotted as a density isosurface in figure 4.1, with the main point being that three dimensionalization is most relevant near the nose of the core as opposed to in the billows. In the nose, interaction between the core and the BBL leads to the lobe-cleft instability, a typical feature of a gravity current head (Simpson, 1972; Simpson and Britter, 1979). The instability is generated when the gravity current head (or the trapped core in this case) enters into the light fluid, resulting in flow separation such that the dense fluid is lifted up and over a small portion of light fluid that is trapped on the BBL due to the no-slip condition. Hartel et al. (2000a,b) found that the stagnation point of the flow is located upstream of the foremost point of the current in the vicinity of the bottom boundary, and the hyperbolic flow pattern and unstable stratification between the nose and the stagnation point are jointly responsible for the generation of the lobe-cleft instability.

We shall mention that although heavy fluid overlying light fluid is also responsible for the generation of the Rayleigh-Taylor instability, the latter is originally formulated when the fluids are initially not moving. In the lobe-cleft set up, Hartel et al. (2000a,b) showed that the flow from the advancing gravity current also plays a role in setting the growth rate and 'shape' of the instability that develops.

The generating mechanism of the lobe-cleft instability in the present work is similar to that in Venayagamoorthy and Fringer (2007, see figures 2 and 3), where a gravity current head is formed as a result of the wave shoaling onto a shelf. We should point out though, due to limited resolution and different model setup (a linear stratification), other features (in particular, the Kelvin-Helmholtz billows) are absent from their simulation results. 


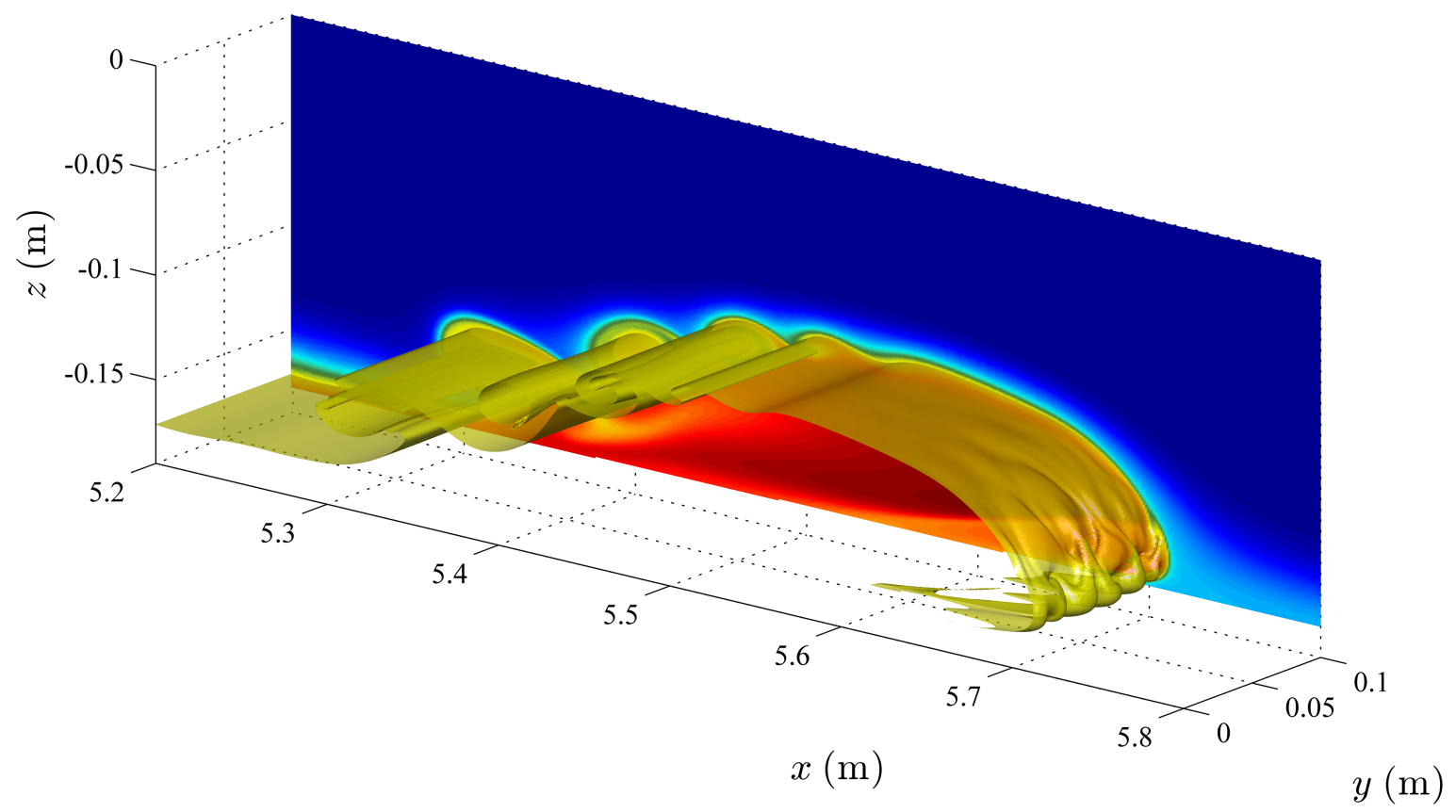

Figure 4.1: Density isosurface corresponding to $\rho=1$ showing the 3D structure of the shoaling wave at $t=42 \mathrm{~s}$. The vertical slice in the background shows the shaded density profile in the $x-z$ plane at $y=0.1 \mathrm{~m}$.

As shown in figure 4.1, while the 3D structure of the flow appears at the foremost part of the core at $t=42 \mathrm{~s}$, the billows at the back of the wave still maintain a 2D structure and behave similar to those in the $2 \mathrm{D}$ case. However, the density profile in figure 4.2 suggests that this is no longer the case at $t=44 \mathrm{~s}$. As the wave propagates, the disturbance due to the lobe-cleft instability develops rapidly and eventually reaches the billows, altering the structure of these billows. At the same time, the shear instability has begun to destroy the billows and the core, while direct interaction between the billows and the BBL has taken place. The figure shows that between $x=5.6 \mathrm{~m}$ and $5.7 \mathrm{~m}$, two billows are starting to overlap with each other, whereas between $x=5.5 \mathrm{~m}$ and $5.6 \mathrm{~m}$, a larger billow has formed due to the interaction of two billows at an earlier time. Near $x=5.5 \mathrm{~m}$, deformation of the pycnocline indicates that the original billows have been swept into the BBL.

The spanwise structure of the flow field at $t=44 \mathrm{~s}$ is shown in figure 4.3 , where density variation in the spanwise direction is found in all panels. Several lobe-and-cleft patters are clearly seen in panel $(e)$, indicating that the lobe-cleft instability is fully developed and three dimensionalized. Note that the $3 \mathrm{D}$ domain has a width of $0.1 \mathrm{~m}$, with periodic 


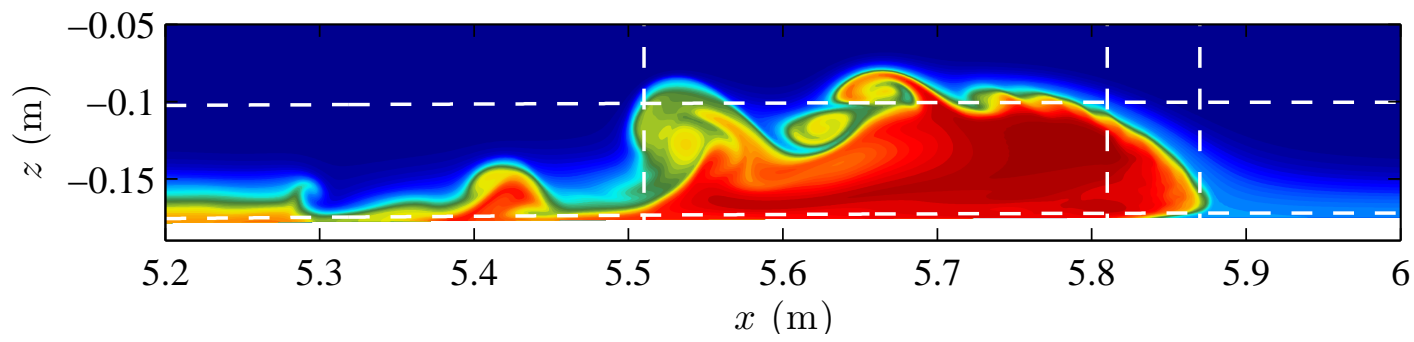

Figure 4.2: Spanwise average of the density field at $t=44 \mathrm{~s}$. Cross section views of the density profile corresponding to the dashed lines are given in figure 4.3.

$(a)$

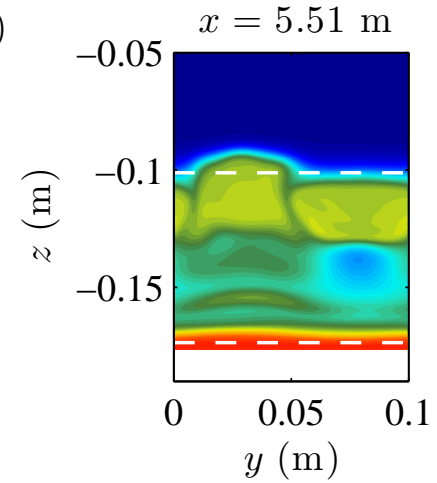

$(b)$

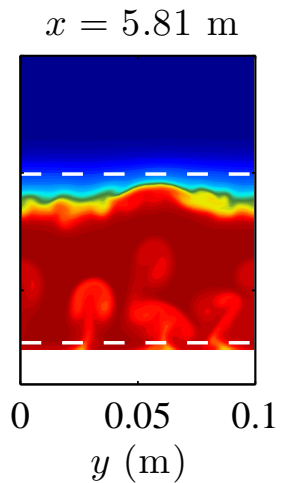

$(c)$

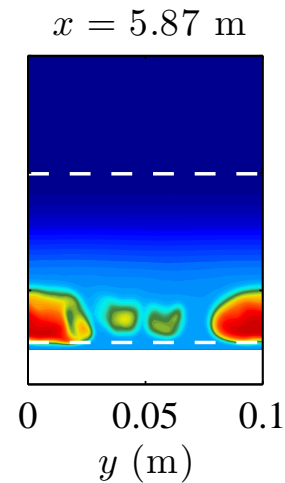

$(d)$

$z=-0.100 \mathrm{~m}$

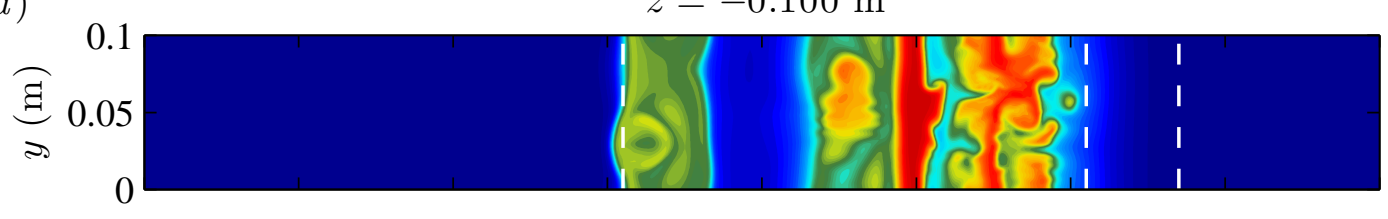

$(e)$

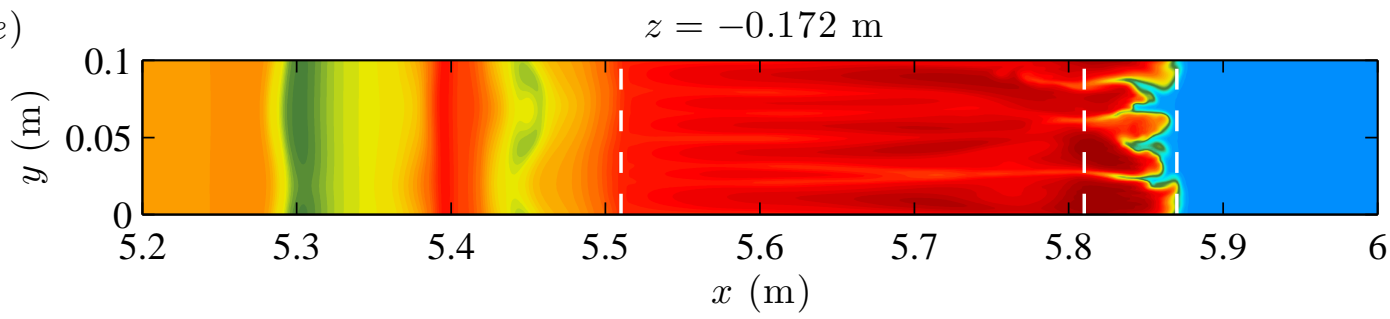

Figure 4.3: Cross section views of the density field at $t=44 \mathrm{~s}$, with the $y$ - $z$ plane views showing $(a)$ the billows, $(b)$ the core's head and $(c)$ nose, and the $x$ - $y$ plane views showing $(d)$ the high shear region at the edge of the wave and $(e)$ the bottom boundary layer. The dashed lines indicate the corresponding locations of the planes in different views. 
boundary conditions used in the spanwise direction. Also visible in panel $(e)$ are the tails of the clefts left behind the initial instability. The light fluid trapped in the BBL is lifted up along these tails, generating vortices as it enters into the heavier fluid in the core (shown in panel $(b))$. Note that the 'mushroom caps' in panel $(b)$ are similar to those found in the Rayleigh-Taylor instability.

At the same time, the Kelvin-Helmholtz billows have also developed a fully 3D structure. In particular, panels $(a)$ and $(d)$ show that the billow at $(x, z)=(5.51,0.1) \mathrm{m}$ has an asymmetric structure across the spanwise direction, while in panel $(e)$ evidence of interaction of billows with the BBL is found between $x=5.3 \mathrm{~m}$ and $5.5 \mathrm{~m}$. Though the original billows have already been swept into the BBL, the spanwise structure on the BBL is still clearly visible.

The vertical velocity profile at $t=44 \mathrm{~s}$ is shown in figure 4.4 . Recall that for a typical ISW of elevation, upward flow is found in front of the wave crest, whereas downward flow is found at the back of the crest (for example, see figure 3.9). In panel $(a)$, while positive vertical velocity is found in front of the wave crest as expected, the area of negative vertical velocity behind the wave crest is broken up into several parts by the billows. Strong positive vertical velocity is found in these billows. In particular, the magnitude of the upward velocity in the billow between $x=5.5 \mathrm{~m}$ and $5.6 \mathrm{~m}$ is much larger than that found in front of the wave crest. This is a clear indication that the shear instability is starting to destroy the wave.

The spanwise structure of the vertical velocity profile shown in panels $(b)-(e)$ is similar to that of the density field (see figure $4.3(d)-(e)$ ). Lobe-and-cleft patterns are clearly seen in panels $(d)$ and $(e)$. A comparison between these two plots and figure $4.3(e)$ suggests that upward velocity is found in the clefts, whereas downward velocity is found in the lobes. This is because the light fluid trapped in the BBL is escaping through the clefts, while the heavy fluid in the lobes is sinking. Such a flow pattern necessarily generates velocity shear between the lobes and clefts. Because there is density stratification between each lobe and cleft, when the shear stress is strong enough, instability is initialized. In fact, the individual vortices in the 'mushroom caps' found in figure $4.3(b)$ are quite similar to Kelvin-Helmholtz billows.

On the other hand, panels $(b)$ and $(c)$ show that asymmetric structure in the spanwise direction is found in the billow between $x=5.5 \mathrm{~m}$ and $5.6 \mathrm{~m}$. Similar structure is also found in the billows that is formed earlier, which has been absorbed into the BBL between $x=5.3 \mathrm{~m}$ and $5.5 \mathrm{~m}$ as evident in panels $(d)$ and $(e)$. 

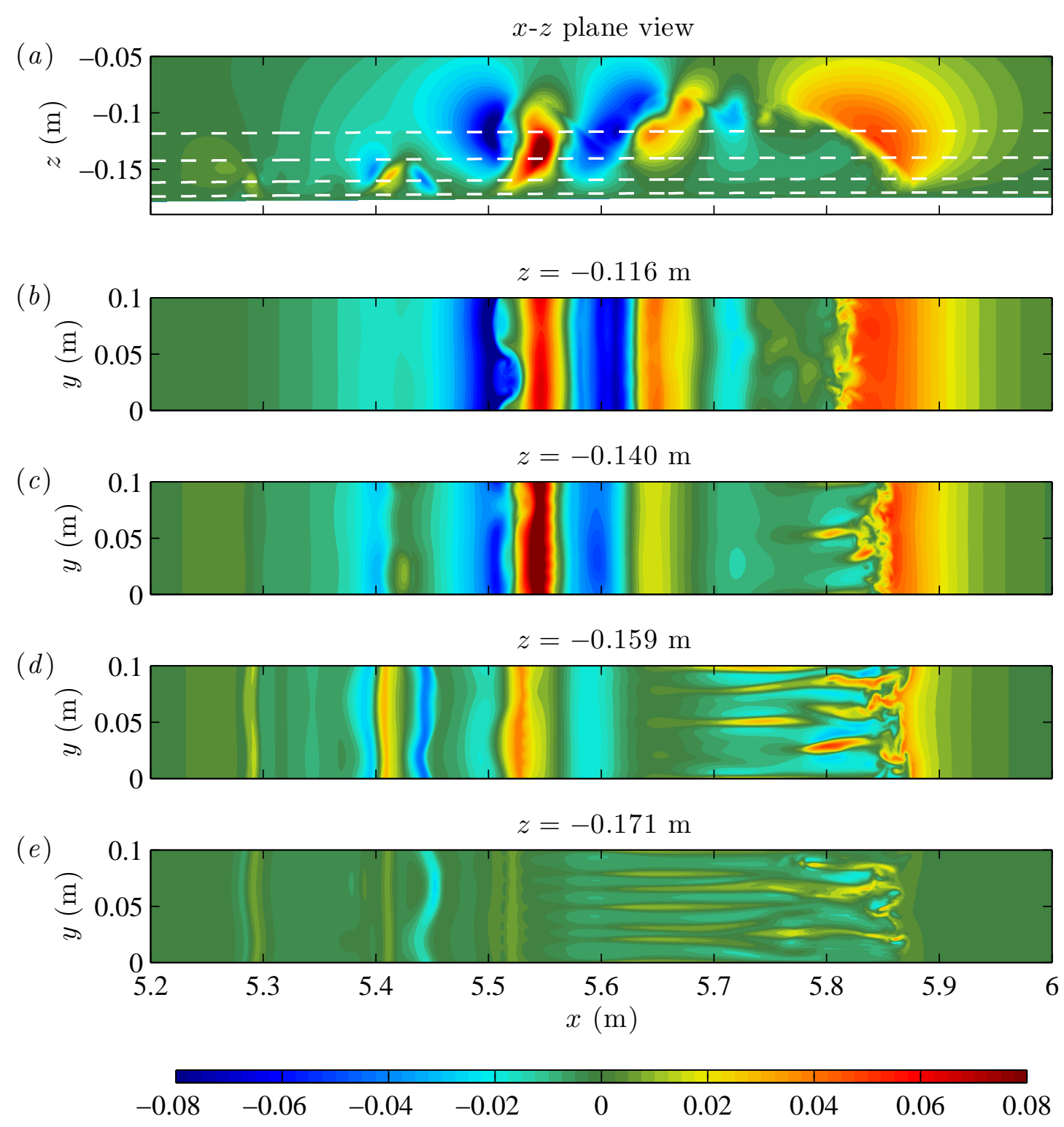

Figure 4.4: Vertical velocity profile at $t=44 \mathrm{~s}$. Panel $(a)$ : spanwise average. Panels $(b)$ $(e): x-y$ plane views corresponding to the horizontal layers indicated by the dashed lines in panel $(a)$. Note that due to the topography of the bottom boundary and the mapping used in the spectral method, these layers are not exactly parallel to the $x-y$ plane in the physical domain. Here, the bottom boundary is located at $z \approx-0.175 \mathrm{~m}$ with an average slope of $0.46 \%$. The color axis is saturated at $\pm 8 \mathrm{~cm} / \mathrm{s}$ with downward velocity shown in blue and upward velocity shown in red. 


\subsubsection{Shoaling Behavior and Boundary Layer Interaction}

In terms of spanwise average, the evolution of the density field during the wave's shoaling is similar to that of the 2D flow, and hence is not shown here. Instead, the standard deviation of the kinetic energy field in the spanwise direction is given in figure 4.5. For a Boussinesq fluid, the kinetic energy $E$ of each fluid particle in the flow field is determined from its local velocity such that

$$
E=\rho_{0}\left(\frac{1}{2} \boldsymbol{u} \cdot \boldsymbol{u}\right) .
$$

The standard deviation shown in figure 4.5 measures the variation of the kinetic energy in the $y$-direction from its spanwise average. It gives an indication of the degree of threedimensionalization of the flow field. A zero spanwise variation in the kinetic energy means that the flow remains two-dimensional, whereas in a highly three-dimensionalized flow field, the kinetic energy carried by each fluid particle deviates from the mean value largely.

Figure 4.5 shows that highlights of the standard deviation of the kinetic energy field are found in the core's nose and edge, suggesting that three-dimensionalization of the flow field takes place in the lobe-cleft region and the Kelvin-Helmholtz billows where instability are developing. The figure also suggests that the 3D structure develops rapidly. In panel (a), the spanwise variation of the kinetic energy is found close to zero in most of the flow field, except in the core's nose where the lobe-cleft instability is initiated. In panel $(b)$, as the instability develops and the wave propagates, 3D structure is found in the BBL and the wave front above the core. In the mean time, three-dimensionalization also occurs in the high shear region at the core's edge, as the shape of the billows becomes visible. In panel $(c)$, as the lobe-cleft instability and the Kelvin-Helmholtz instability further develop, the entire core region is eventually fully three-dimensionalized. This observation is consistent with the density profile shown in figure 4.3. In particular, the brightest portions of the plot are found in the core's nose and the billow between $x=5.5 \mathrm{~m}$ and $5.6 \mathrm{~m}$.

During the wave's shoaling process, two mechanisms contribute to the boundary layer interaction, namely the lobe-cleft instability and the billow-BBL interaction. Both of these mechanisms lead to increased the bottom shear stress. The evolution of the bottom shear stress is plotted in figure 4.6, featuring a comparison between the 2D case and the 3D case. Here the magnitude of the bottom shear stress is measured by

$$
\left\|\boldsymbol{t}_{s}\right\|=\sqrt{t_{x}^{2}+t_{y}^{2}},
$$

where $t_{x}$ and $t_{y}$ are defined in equation (2.51). This figure shows that at $t=40 \mathrm{~s}$, the bottom shear stress curves produced from the 2D and 3D simulations are almost identical, 

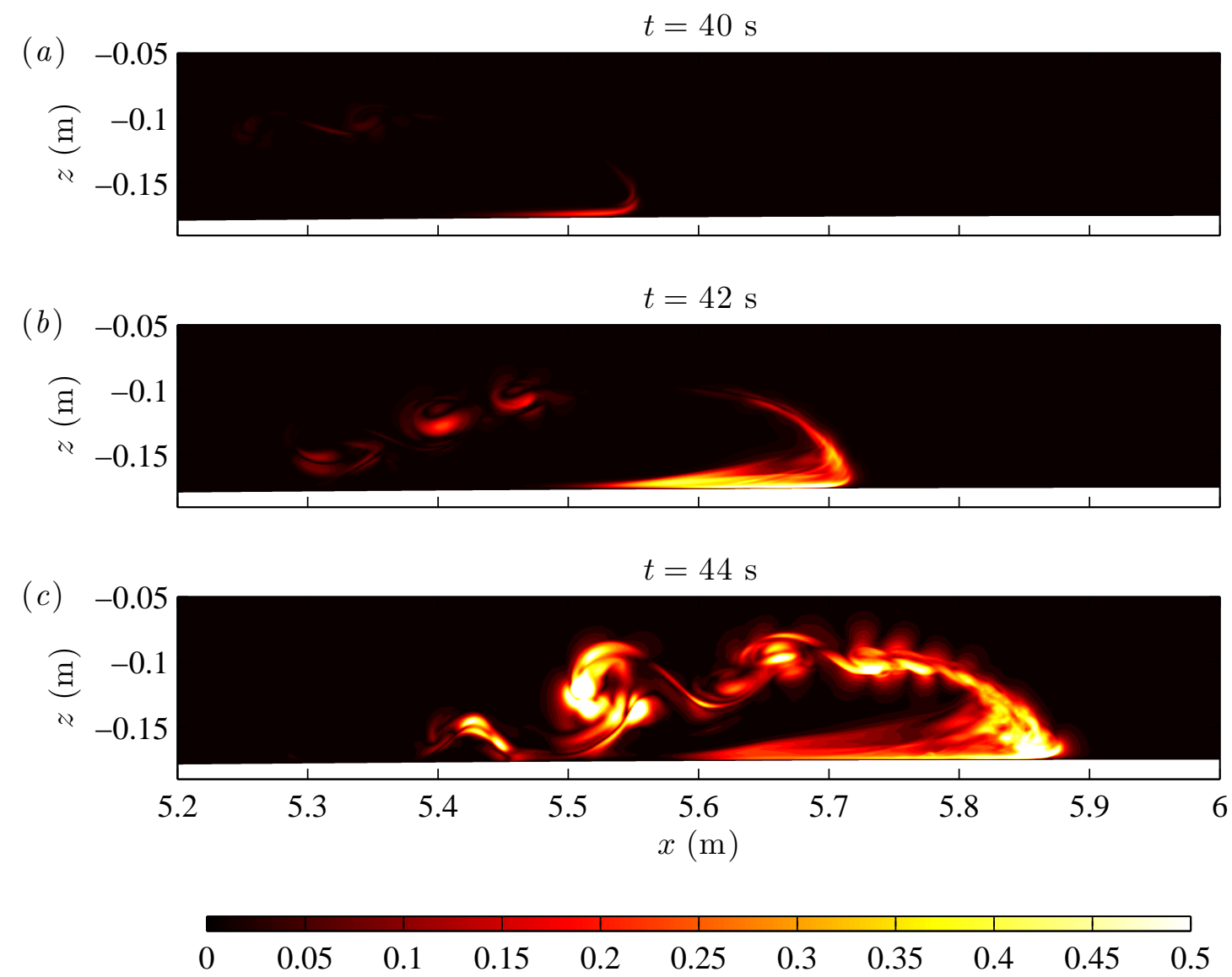

Figure 4.5: Normalized standard deviation of the kinetic energy field in the spanwise direction at $(a) t=40 \mathrm{~s},(b) t=42 \mathrm{~s}$ and $(c) t=44 \mathrm{~s}$. The dark regions represent a value of zero, while the highlights are saturated at $50 \%$ of the maximum value found in these plots.

because at this time most of the flow structure still remains two-dimensional as suggested in figure $4.5(a)$. As the 3D structure develops, significant increase of the bottom shear stress in the lobe-cleft region is found in the 3D simulation, whereas the maximum bottom shear stress in the $2 \mathrm{D}$ case remains almost the same throughout the shoaling process. At the same time, increase of bottom shear stress due to billow-BBL interaction is found at the back of the wave, though there is no quantitative difference between the $2 \mathrm{D}$ and the $3 \mathrm{D}$ case. From this figure we conclude that the three-dimensionalization of the flow field does contribute to the increased bottom shear stress, primarily through the generation of 
$(a)$

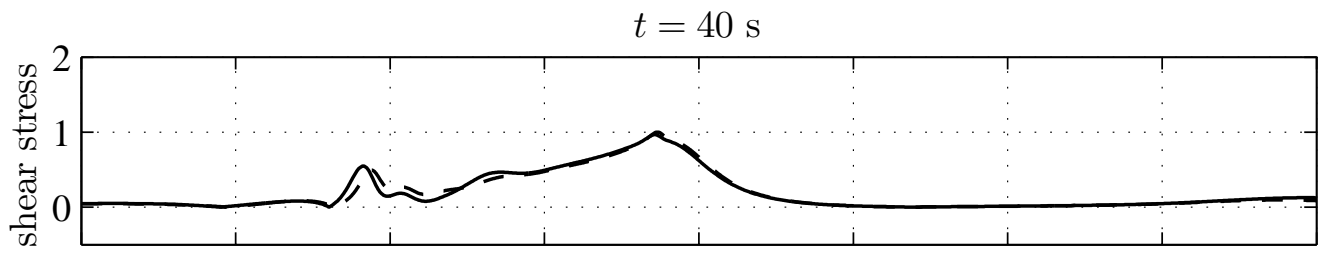

$(b)$

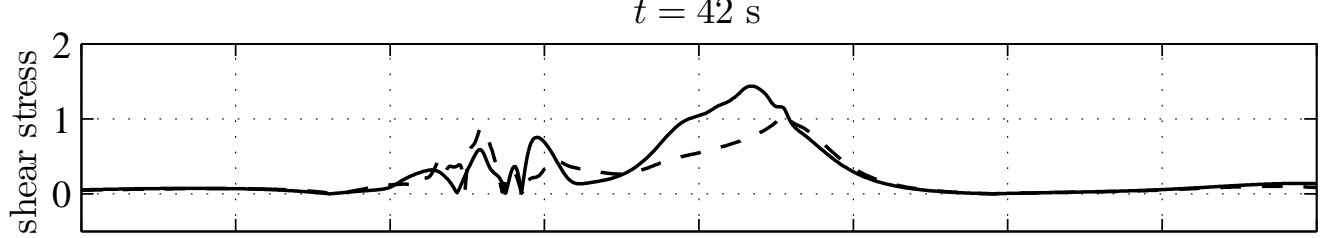

$(c)$

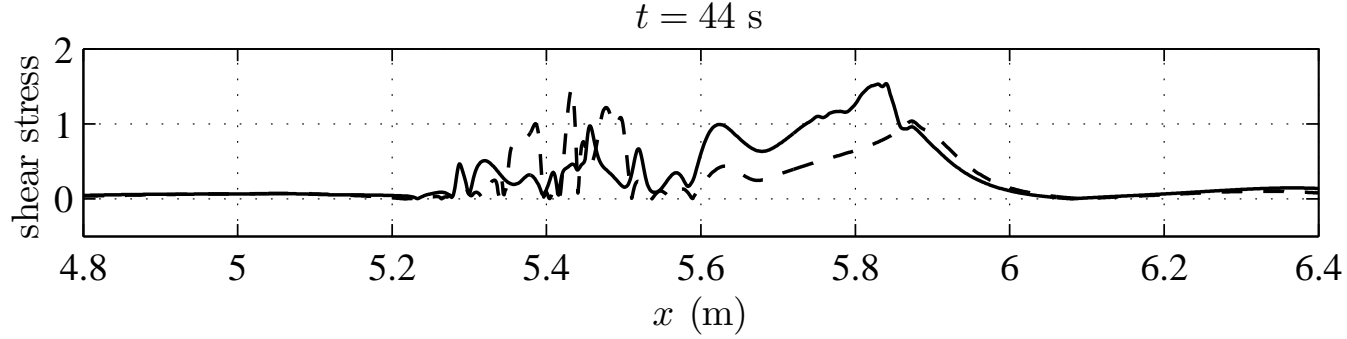

Figure 4.6: Normalized bottom shear stress at $(a) t=40 \mathrm{~s},(b) t=42 \mathrm{~s}$ and $(c) t=44 \mathrm{~s}$. Solid lines represent results obtained from the 3D simulation, and dashed lines represent results obtained from the $2 \mathrm{D}$ simulation. Spanwise averages are used in the 3D case.

the lobe-cleft instability.

More detailed bottom shear stress plots of the 3D simulation are given in figure 4.7, where both the streamwise and spanwise components are shown. While the overall bottom shear stress is dominated by the upstream-directed (positive) stress due to wave passage, perturbations are also observed in the figure. The lobe-and-cleft patterns are clearly visible in both the streamwise and the spanwise directions, and interaction between the billows and the BBL is also evident. In particular, consistent upstream shear stress is observed in the lobe-cleft region, whereas at the back of the wave, the magnitude of the shear stress introduced by the billows develops rapidly over time, and eventually becomes twice as strong as that in the lobe-cleft region (shown in panel $(b)$, between $x=5.6 \mathrm{~m}$ and 5.7 $\mathrm{m})$. On the other hand, downstream-directed (negative) stress is found behind the wave, whose magnitude is a fraction of the upstream-directed stress. At the same time, the spanwise component of the shear stress plays a secondary role in general, as suggested by 


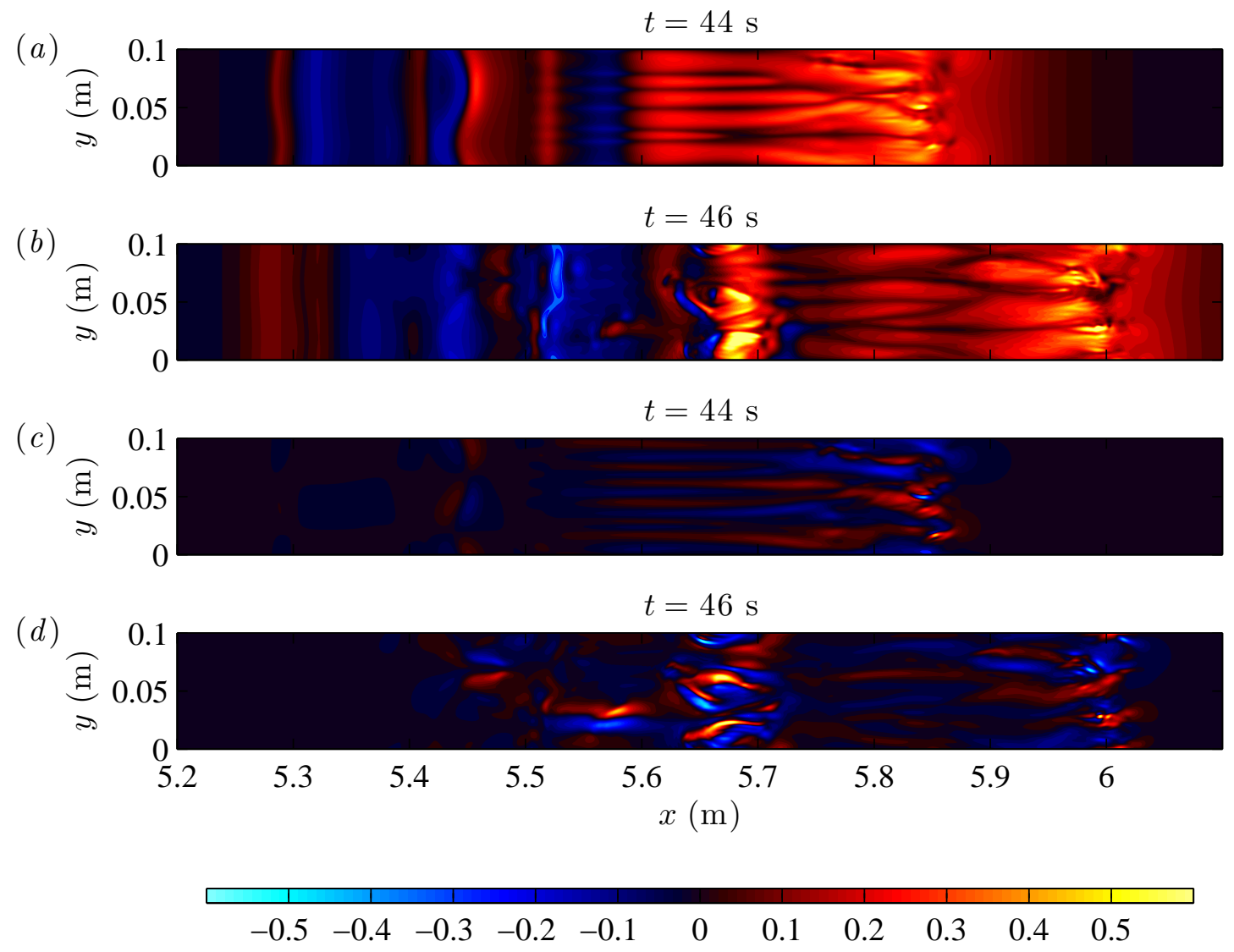

Figure 4.7: Normalized shear stress histories on the bottom boundary in $(a)-(b)$ the streamwise direction and $(c)-(d)$ the spanwise direction. For all subplots, the color axis is saturated at $\pm 60 \%$ of the maximum along-hill shear stress observed in panel $(b)$, with positive values shown in red (hot), negative values shown in blue (cold) and intermediate values shown in black.

the comparison given in figure 4.7. An exception is that at $x=5.65 \mathrm{~m}, t=46 \mathrm{~s}$, the spanwise shear stress is about half of the magnitude as the streamwise shear stress (shown in panel $(d))$.

The combined effect of the lobe-cleft instability and the billow-BBL interaction has significant environmental implications. In the near-coastal region, such mechanism is likely to contribute to onshore sediment movement during the wave passage followed by offshore movement afterwards (Bourgault et al., 2014). While the bottom shear stress mobilizes the 
sediment within the BBL, the turbulence introduced by the instabilities pumps material out of the boundary layer. For example, field measurements by Richards et al. (2013) suggest that the trapped core of waves of elevation can produce strong vertical shear while interacting with the $\mathrm{BBL}$, as evident at $t=16: 19$ in their figure 9. In nature, waves rarely exist as single waves, and thus motion initialized by the first wave could modify the subsequent waves in the wave train, magnifying the transport and mixing mechanism. In addition, for an organic sediment bed, the boundary layer interaction will also contribute to the dissolved oxygen transfer and affect the marine ecosystem by mechanisms similar to 'scarring by coherent structures' proposed in Scalo et al. (2012). 


\subsection{The Medium Pycnocline Case}

In the simulation labeled as Medium, the pycnocline is located at $z_{0}=-0.16 \mathrm{~m}$, which is $1.5 \mathrm{~cm}$ above the crest of the bottom topography. Since the bottom boundary does not reach into the pycnocline, the ISW does not behave like a gravity current as it shoals onto the shelf. Instead, the wave maintains a solitary wave-like form throughout the shoaling process and adjusts to the elevated bottom boundary by changing its shape. The 2D simulation suggests that, unlike the Base Case where the instabilities are generated during the shoaling, in the Medium pycnocline case the instabilities occur behind the main wave after the shoaling. During the de-shoaling process, the core which had formed in the original wave breaks up and the fluid ejected from the core forms a vortex-rich region near the down-sloping portion of the shelf, in which the flow is highly turbulent and the bottom shear stress is extremely strong. In front of the remnants of the core, a new solitary wave is formed and propagates away, in a similar manner to the original wave.

The 3D dynamics of the Medium pycnocline case is discussed in this section. In the 3D simulation, the wave behaves in a slightly different manner from the corresponding 2D simulation. In particular, during shoaling a lobe-cleft instability occurs on the leading edge of the forming core. This occurs despite the fact that the overall flow behaviour is better described as a solitary wave as opposed to a gravity current. In the remainder of this section, the lobe-cleft instability will be examined in detail. The formation and evolution of the vortex-rich region in three dimensions will also be discussed, focusing on the spanwise structure of the vorticity field that does not exist in the $2 \mathrm{D}$ simulation.

\subsubsection{Lobe-Cleft Instability}

The density profile of the wave as it reaches the top of the bottom shelf is plotted in figures 4.8 and 4.9. The spanwise average of the density field shown in figure 4.8 suggests that the overall structure of the wave is similar to that in the 2D simulation. More specifically, there is no shear instability during the shoaling process while the trapped core has already begun to overturn toward the left. On the other hand, the cross section views shown in figure 4.9 suggest that the lobe-cleft instability is generated. The lobe-and-cleft patterns found in panel $(d)$ is very similar to those seen in figure $4.3(e)$, while in panel $(b)$ the 'mushroom caps' are also observed in the core's head. In addition, the lobe-cleft structure in the core's nose shown in panel $(c)$ also leads to disturbance found at the edge of the core, such that a spanwise structure is seen near $z=-0.13 \mathrm{~m}$ in panel $(b)$. After the wave passage, the BBL remains in a three-dimensional manner, as shown in panel $(a)$. 


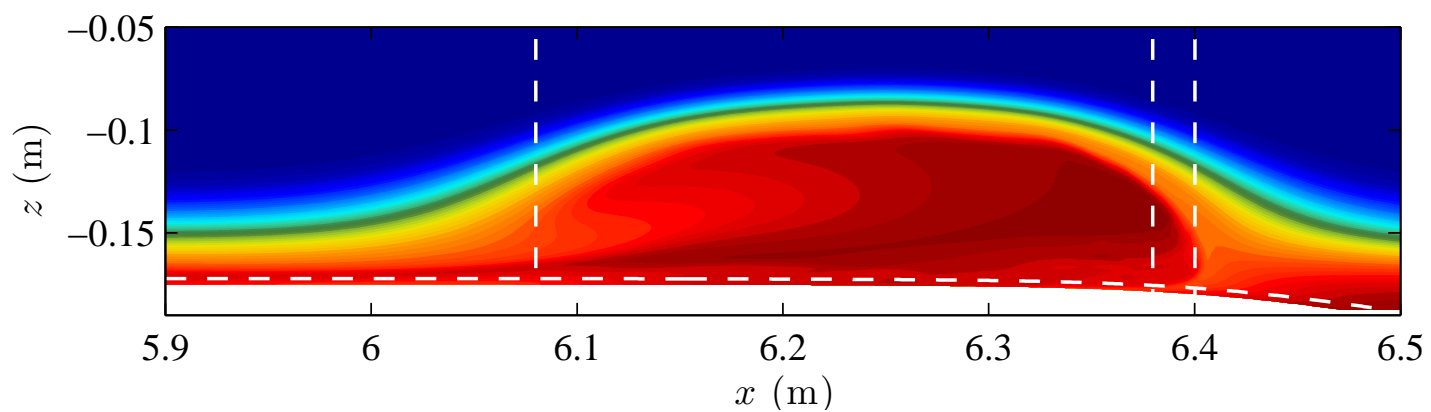

Figure 4.8: Spanwise average of the density field at $t=50 \mathrm{~s}$. Cross section views of the density profile corresponding to the dashed lines are given in figure 4.9.

(a)

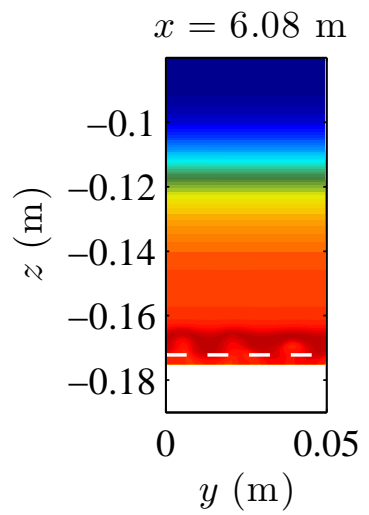

(b)

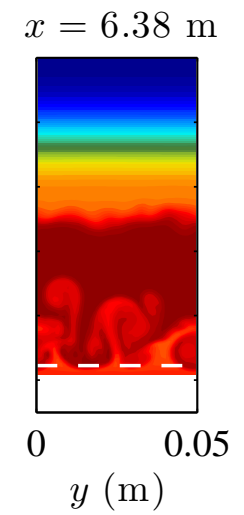

(c) $\quad x=6.40 \mathrm{~m}$

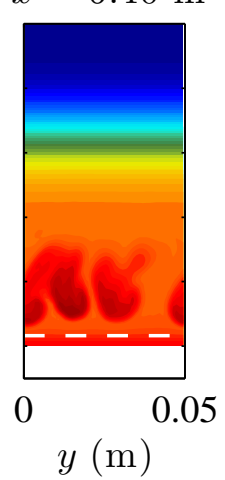

(d)

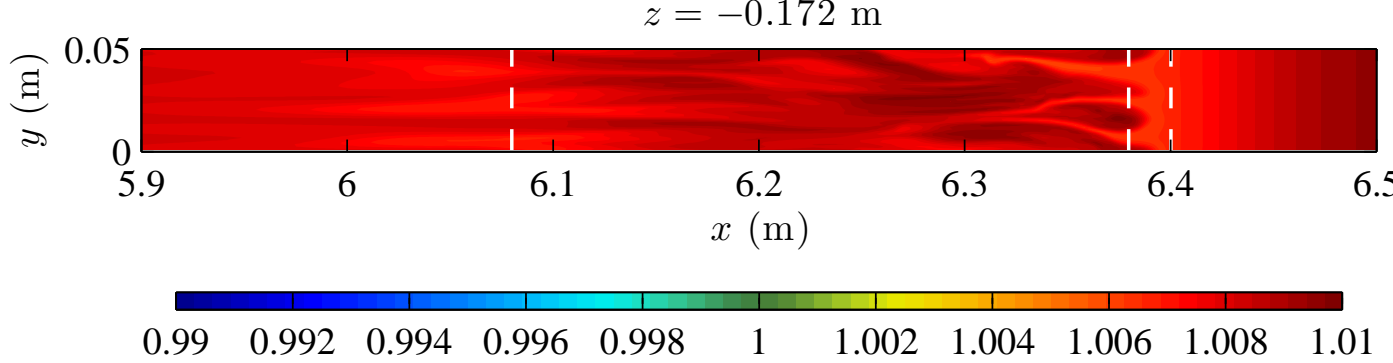

Figure 4.9: Cross section views of the density field at $t=50 \mathrm{~s}$ corresponding to the locations indicated by the dashed lines in figure 4.8, with $(a)-(c)$ the $y$ - $z$ plane views showing the lobe-cleft instability, and $(d)$ the $x-y$ plane view showing the boundary layer located at $3 \mathrm{~mm}$ above the bottom boundary. Note that the bottom boundary layer is not exactly parallel to the $x-y$ plane due to the topography. 
This observation suggests that the lobe-cleft instability does not only exist in gravity currents, but also in shoaling ISWs of elevation. Although figure $4.9(d)$ shows that density gradient exists in the BBL, the overall color tone suggests that the density difference between the heavy and light fluids is only approximately $0.2 \%$ (as a reference, the colorbar is reproduced in the figure). With such a small density difference, the resulting buoyancy force is not sufficiently large to provide the trigger for the instability. Hence, this phenomenon clearly indicates that it is the advancing nature of shoaling waves or gravity currents that acts as the driving mechanics for the generation of the lobe-cleft instability, whereas heavy fluid overlying light fluid is only a necessary but not sufficient condition. In Hartel et al. (2000a,b), direct numerical simulations were performed of a gravity current head. Despite the different model setup, the lobe-cleft instability was observed and the same conclusion has been drawn. In particular, a linear stability analysis is provided in section 4 of Hartel et al. (2000a) to explain this phenomenon.

\subsubsection{The Vortex-Rich Region}

In three dimensions, an important variable in the discussion of vorticity dynamics is enstrophy $Q$, which measures the overall strength of the vorticity field in all directions. It is defined in a way similar to the kinetic energy:

$$
Q=\rho_{0}\left(\frac{1}{2} \boldsymbol{\omega} \cdot \boldsymbol{\omega}\right)
$$

The evolution of the enstrophy during the wave's de-shoaling process is given in figure 4.10. Similar to the 2D case (figure 3.12), baroclinic vorticity is found along the wave crest and the core's edge, while vorticity production due to acceleration of the fluid during the wave passage is found along the BBL. The differences from the $2 \mathrm{D}$ vorticity field are also notable. First of all, with the presence of vortex stretching (the term $(\boldsymbol{\omega} \cdot \nabla) \boldsymbol{u}$ in equation (4.1)) which does not exist in a 2D flow field, the flow appears to be more turbulent and the instability is generated more rapidly. The dissipation of energy also occurs within a shorter period of time, because in the $2 \mathrm{D}$ case the vortices stay more coherent. Secondly, in panel $(b)$ the formation of billows is observed at the edge of the core, indicating that the shear stress is stronger in the $3 \mathrm{D}$ case. This phenomenon is similar to what is observed in figure 3.15, where the increase of shear stress is due to perturbation of the BBL caused by the bottom corrugations. For the $3 \mathrm{D}$ case, perturbation of the BBL comes from the lobe-cleft instability. Last but not least, panel $(c)$ suggests that systematic boundary layer separation does not occur in the 3D case (although it may occur locally), because the lobe-cleft instability has already destroyed the BBL during the wave's shoaling. 

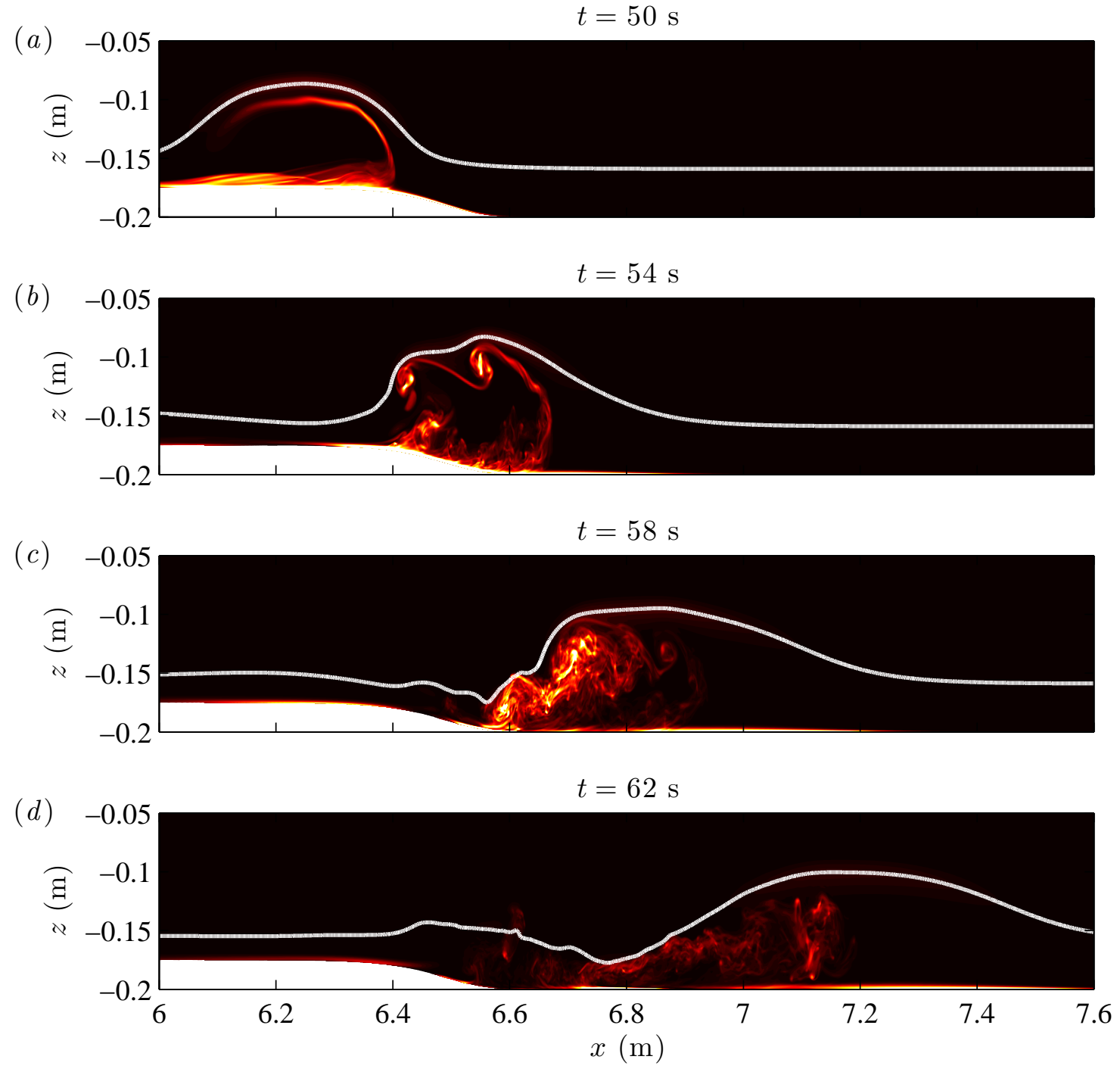

Figure 4.10: Spanwise average of the normalized enstrophy plots showing the wave's deshoaling process. The brightness in these plots is proportional to the magnitude of the enstrophy field. The pycnocline is indicated by the solid curves. 

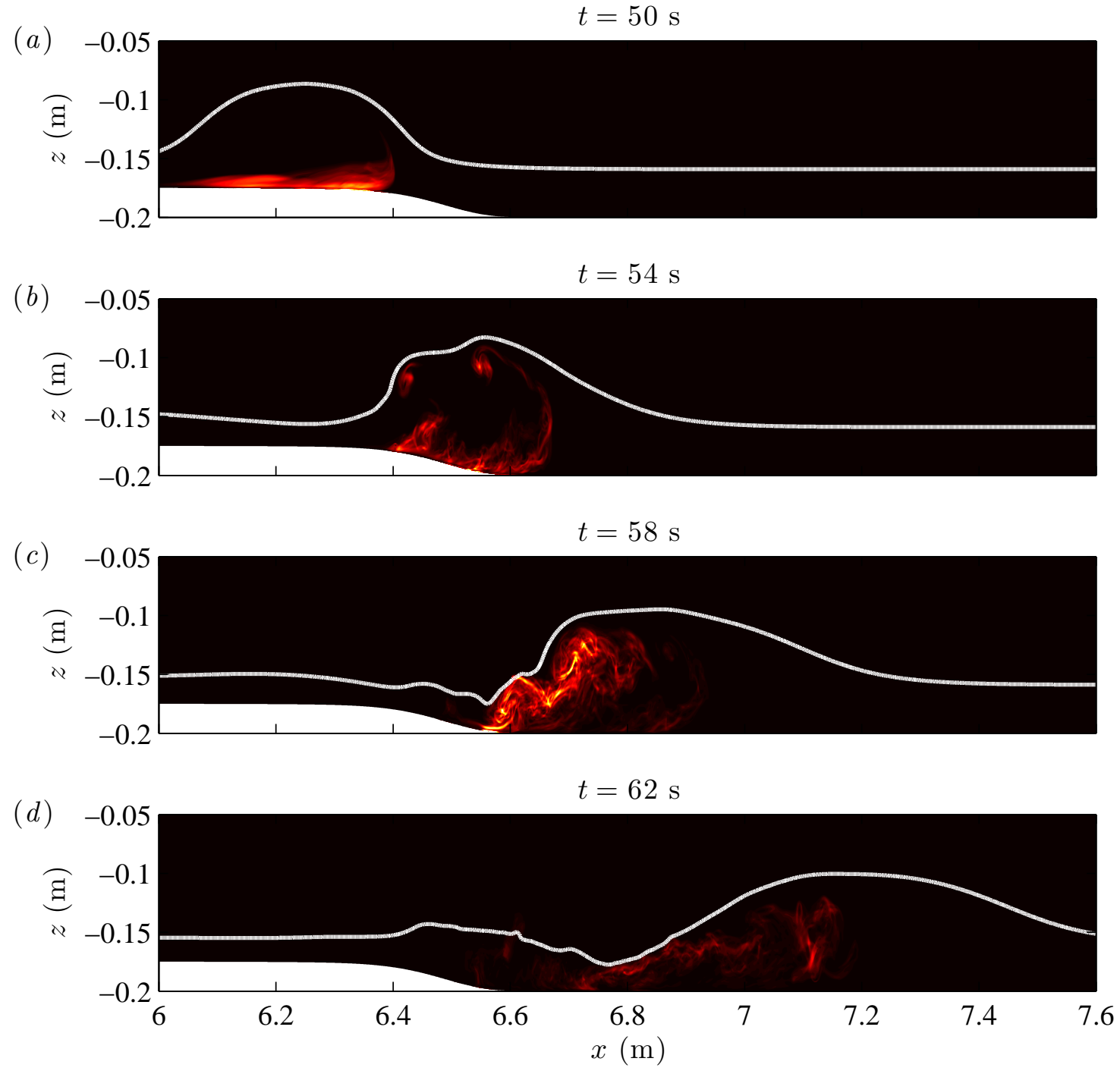

Figure 4.11: Spanwise average of the normalized difference between the enstrophy and the $y$-component of the vorticity (i.e. the quantity $Q_{y}$ defined in equation (4.6)) corresponding to figure 4.10 . 
The three-dimensionality of the flow field can be described by the strength of the $x$ and $z$ components of the vorticity field, because in a 2D flow the only non-zero component of the vorticity field is the $y$-component. Here, we define $Q_{y}$ to be the difference between the enstrophy and the $y$-vorticity:

$$
Q_{y}=Q-\frac{1}{2} \rho_{0} \omega_{y}^{2}=\frac{1}{2} \rho_{0}\left(\omega_{x}^{2}+\omega_{z}^{2}\right) .
$$

In this equation, the subscripts denote the individual components of the vorticity field, i.e. $\boldsymbol{\omega}=\left(\omega_{x}, \omega_{y}, \omega_{z}\right)$. Corresponding to the enstrophy plot in figure 4.10 , the evolution of $Q_{y}$ is plotted in figure 4.11. In a way similar to the standard deviation plot of the kinetic energy, in this figure the bright regions indicate a non-zero value of the $x$ and $z$ components of the vorticity field, which contributes to the spanwise structure of the flow field, whereas the dark regions consist of mainly the $y$-component of the vorticity field (or no vorticity at all), so that the flow is two-dimensional. In figure 4.11, the baroclinic vorticity along the wave crest and the core's edge and the vorticity production due to viscosity in the BBL are no longer seen, suggesting that these types of vorticity are mainly associated with the mean flow in the along-topography direction. On the other hand, bright regions are found in the lobe-cleft region (panel $(a)$ ) and the vortex-rich region (panel $(c)$ ), indicating that most of the 3D flow occurs in these regions. Additionally, although the baroclinic vorticity which leads to the shear instability and billow formation seen in figure $4.10(b)$ is generated by the $y$-vorticity, three-dimensionalization of these billows is found in panel $(b)$.

Figure 4.12 shows the $z$-component of the vorticity field at the slice $2 \mathrm{~mm}$ above the bottom boundary, characterizing the evolution of the BBL during the de-shoaling of the wave. The wave passage can be visualized by the advancing of the high-vorticity region. This region has the largest overall magnitude when the wave is on the top of the shelf (panel $(a), t=50 \mathrm{~s})$, which is a clear indication that the dynamics of the BBL is dominated by the lobe-cleft instability during the wave's shoaling. As the wave propagates down the shelf (panels $(b)$ and $(c)$ ), the magnitude of the $z$-vorticity in the advancing high-vorticity region slowly decreases. Also, the alternating pattern between the positive and negative vorticity seen earlier disappears, and the flow field becomes more unpredictable.

The corresponding along-topography component of the bottom shear stress plots are given in figure 4.13. The lobe-and-cleft patterns are clearly seen in panel $(a)$, where the clefts correspond to the positive vorticity seen in figure $4.12(a)$, and the lobes correspond to the regions of zero or negative vorticity. The largest magnitude of the positive shear stress is found in these lobes. Another feature to notice is that unlike the Base Case (figure 4.7), no down-stream directed stress is observed in this plot, since there are no Kelvin-Helmholtz billows interacting with the BBL in this case. As the wave propagates 
(a)

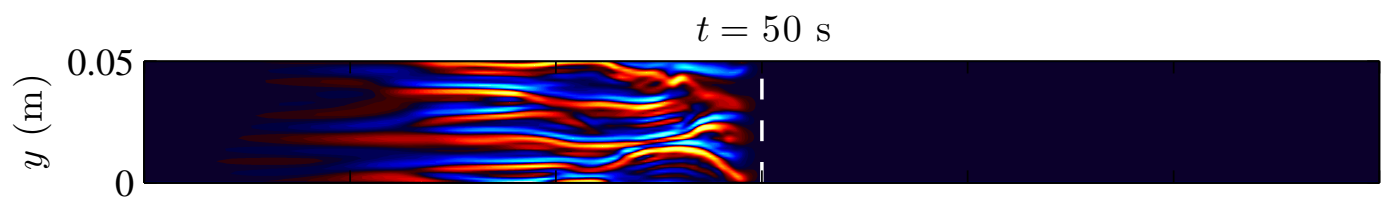

(b)

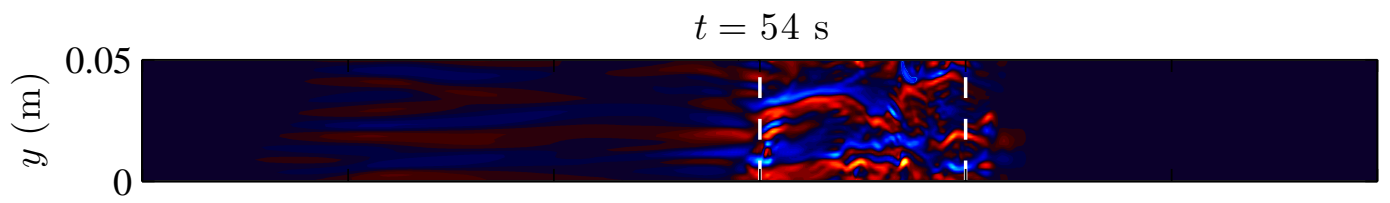

(c)

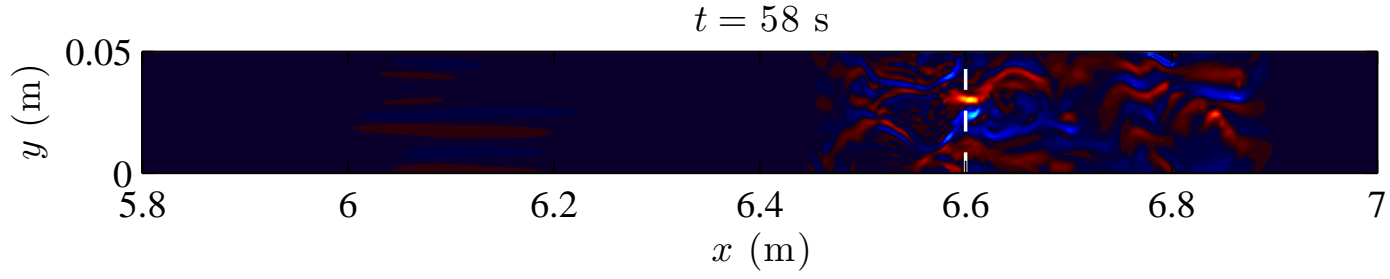

Figure 4.12: Normalized $z$-vorticity plots along the slice $2 \mathrm{~mm}$ above the bottom boundary showing the evolution of the BBL during the de-shoaling of the wave. The positive (negative) vorticity is shown in red (blue) with the magnitude indicated by the brightness. The $x$-vorticity at the slices indicated by the dashed lines is given in figure 4.14.

$(a)$

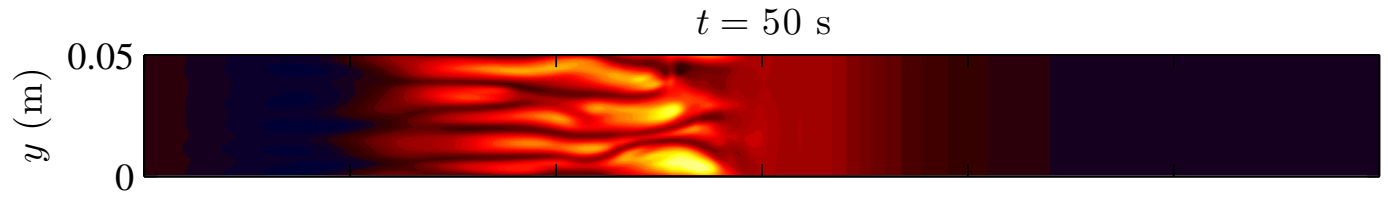

(b)

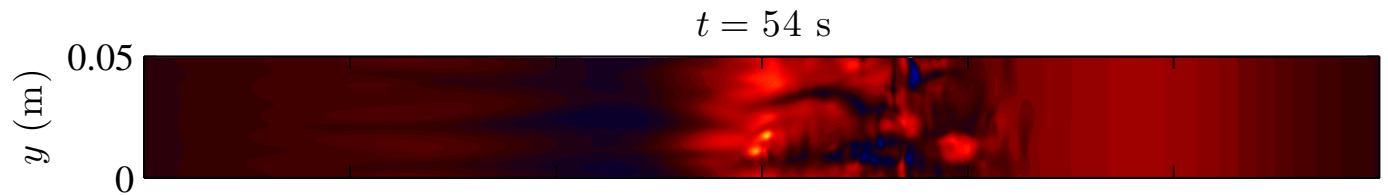

(c)

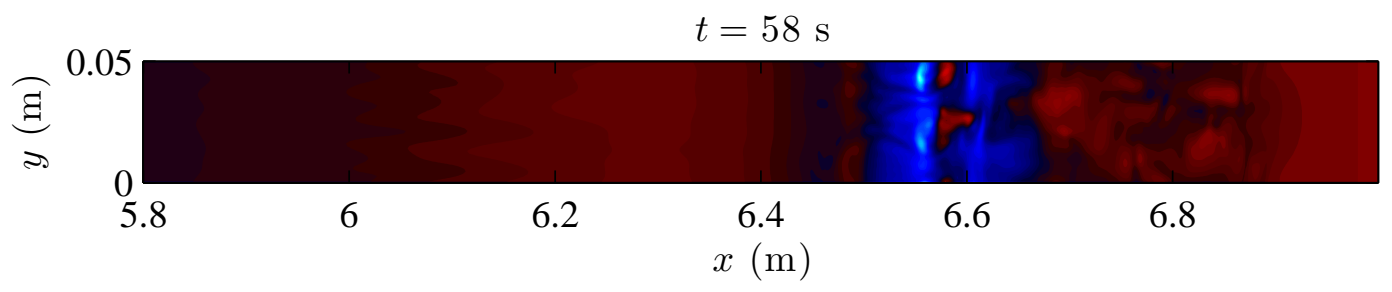

Figure 4.13: Normalized along-topography bottom shear stress. The positive (negative) stress is shown in red (blue) with the magnitude indicated by the brightness. 

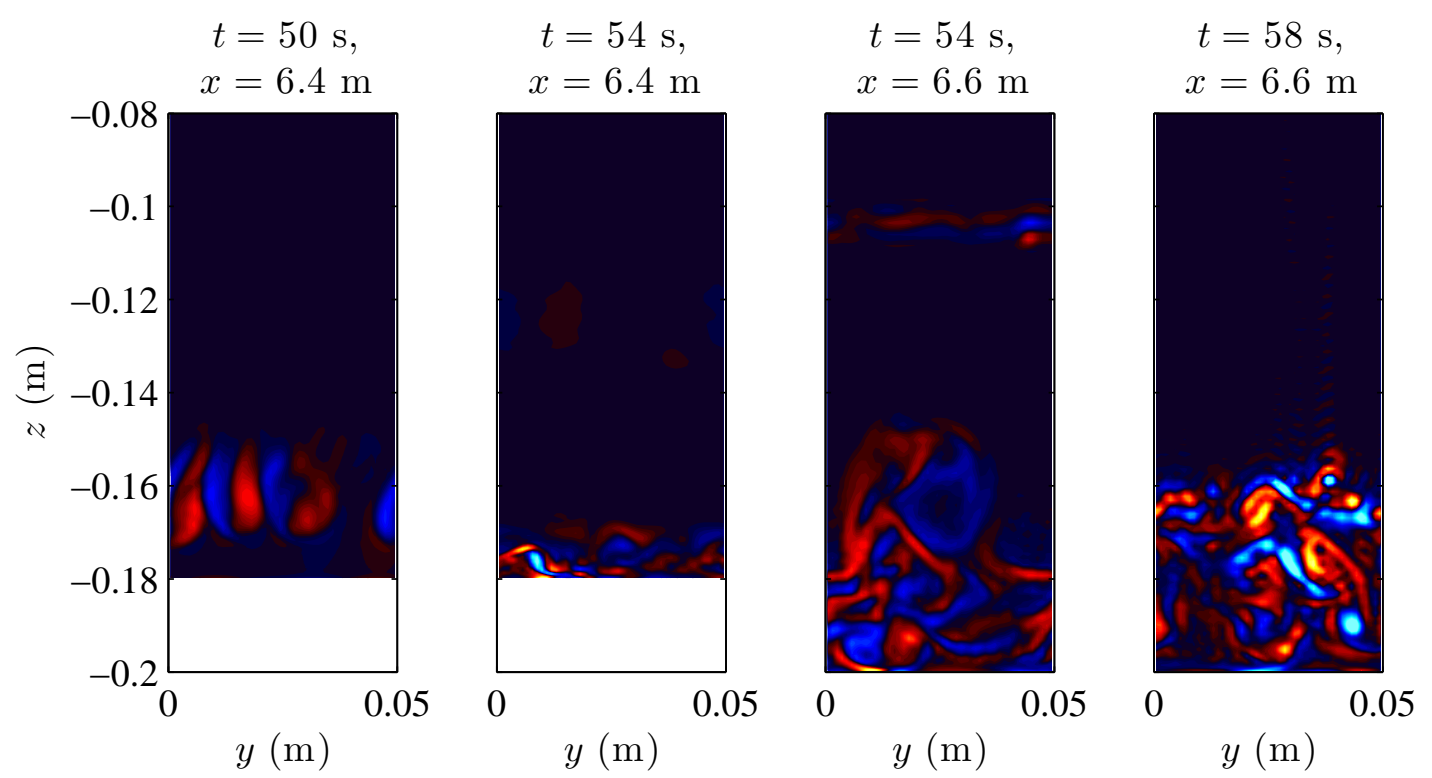

Figure 4.14: Normalized $x$-component of the vorticity field in the $y$ - $z$ plane at $(a) t=50$ $\mathrm{s}, x=6.4 \mathrm{~m},(b) t=54 \mathrm{~s}, x=6.4 \mathrm{~m},(c) t=54 \mathrm{~s}, x=6.6 \mathrm{~m}$ and $(d) t=58 \mathrm{~s}, x=6.6 \mathrm{~m}$, corresponding to the dashed lines in figure 4.12. The color axis is the same as figure 4.12.

down the shelf, the lobe-and-cleft patterns disappear, and the overall magnitude of the shear stress becomes smaller. Down-stream (negative) directed stress eventually appears over the down-slope portion of the shelf in panel $(c)$ during the wave passage, which is similar to the behavior observed in the 2D case (figure 3.13). This suggests that downstream directed flow exists in the BBL. In particular, two bright spots are found in this region of negative stress, indicating that local boundary layer separation is very likely to occur. The spanwise shear stress is not shown here because it only reaches a fraction of the along-topography stress throughout the wave's de-shoaling process, and hence is not important in this case. This is because in the vortex-rich region, most of the spanwise mixing occurs above the BBL.

Four panels of the $x$-vorticity field plots at the slices indicated by the dashed lines in figure 4.12 are given in figure 4.14. In panel $(a)$, the $y$ - $z$ plane is located at $x=6.4 \mathrm{~m}$, corresponding to the nose of the core when the wave reaches the top of the bottom shelf. A comparison with the corresponding density plot, figure $4.9(\mathrm{c})$, suggests that the same patterns are found near $z=-0.16 \mathrm{~m}$, indicating that the lobe-cleft instability does not only contribute to the $z$-vorticity along the BBL, but also the $x$-vorticity in the leading edge of the core. This explains the fact that in the 3D flow the existence of the lobe-cleft 
instability also plays a role in the generation of the shear instability along the core's edge seen in figure $4.10(b)$. As the wave propagates away, the lobe-cleft structure is no longer seen, but vortices still exist on the BBL as shown in panel $(b)$.

The location of the vertical slices shown in panels $(c)$ and $(d)$ corresponds to the vortexrich region $(x=6.6 \mathrm{~m})$. In contrast with panel $(b)$ where a zero vorticity occupies most of the plot, non-zero vorticity is seen in and above the BBL up to $z=-0.14 \mathrm{~m}$ in both of these plots. Moreover, in panel $(c)$ non-zero vorticity is also found in the region of KelvinHelmholtz billow (near $z=-0.1 \mathrm{~m}$ ), despite its small magnitude compared with the rest of the plot. An increase of the overall magnitude of the vorticity field is observed in panel $(d)$, indicating that due to the de-shoaling of the wave, the flow is highly turbulent in this region. Since figure $4.13(c)$ suggests that local boundary layer separation also occurs at this particular point at $t=58 \mathrm{~s}$, we conclude that, in agreement with the result from the $2 \mathrm{D}$ simulation, potential material mixing and sediment movement and resuspension is highly anticipated in this region. The only difference is that in the 3D case, an additional driven mechanism is present, namely the stretching and tilting of the vortex lines, which further contributes to the turbulence of the flow. 


\section{Chapter 5}

\section{Conclusions}

In this thesis we have presented direct numerical simulations of laboratory-scale, fully nonlinear ISWs of elevation propagating over bottom topography, focusing on wave-induced instabilities during the shoaling and de-shoaling processes. The simulations are performed on a mapped rectangular domain with a small-amplitude shelf on the bottom boundary. We focus on flows in a quasi two-layer stratification with a $2 \%$ density difference. Cases with three different locations of the pycnocline are considered. In particular, both 2D and 3D simulations are performed of the Bases Case and the Medium pycnocline cases, and a 2D simulation is performed of the Higher pycnocline case. In addition, a 2D simulation is also performed of the medium pycnocline case with a Rough bottom topography. In all simulations, the initial waves do not reach the breaking limit in deep water, and the trapped cores are formed during the shoaling process.

In the Base Case, the pycnocline is very close to the bottom boundary such that it intercepts the shelf, and the shallowest region is nearly unstratified. As a result, the shoaling wave essentially behaves like a gravity current as it reaches the top of the shelf. The 2D flow is characterized by a spatially growing stratified shear instability in the form of Kelvin-Helmholtz billows at the edge of the core, whereas three-dimensionalization of the flow is more relevant near the nose of the core where the lobe-cleft instability occurs. We shall mention that in the numerical experiments performed by Venayagamoorthy and Fringer (2007), the lobe-cleft instability is also observed but the shear instability is not present. In fact, our 3D simulation suggests that both of these instabilities develop and three-dimensionalize concurrently. In the case of the shear instability, the interaction of the billows and the BBL at the back of the wave leads to strong bottom shear stress. The magnitude of the along-topography component of the shear stress is found to be comparable 
to that due to the lobe-cleft instability, while the spanwise component has a magnitude of at least $50 \%$ of the along-topography component.

In the Medium and Higher pycnocline cases where the bottom shelf does not reach into the pycnocline, the waves maintain a solitary wave-like form throughout the shoaling process and adjust to the elevated bottom boundary by changing their shapes. In these cases the dominant instabilities are generated behind the main wave during the de-shoaling process, in contrast with the Base Case where the instabilities occur during the wave's shoaling. In the Medium pycnocline case, the core formed in the original wave breaks up during the wave's de-shoaling, and the fluid ejected from the core forms a vortex-rich region near the down-sloping portion of the shelf, leading to highly turbulent flow and enhanced bottom shear stress in this region. The magnitude of the bottom stress at this region is found to be negative because of boundary layer separation and has a magnitude as much as three times that of the positive shear stress induced by the wave passage. In front of the remnants of the core, a new, nonbreaking solitary wave is formed and propagates away, in a similar manner to the original wave. In the Higher pycnocline case, the break up of the core still occurs as the main wave propagates down the shelf, but the vortex-rich region is not present. Instead, as the core moves out of the main wave, it fissions into a train of several smaller amplitude, broader ISWs behind the leading wave.

In the $3 \mathrm{D}$ simulation of the Medium pycnocline case, the wave behaves in a slightly different manner from the corresponding 2D simulation during the shoaling, as the lobecleft instability is observed at the leading edge of the core. Given that the overall flow behaviour is better described as a solitary wave as opposed to a gravity current, this phenomenon clearly indicates that it is the advancing nature of the shoaling wave (or the gravity current) that acts as the driving force for the generation of the lobe-cleft instability. This observation agrees with the conclusion drawn in Hartel et al. (2000a,b), in which a linear stability analysis is performed to explain this phenomenon. During the de-shoaling process, with the presence of vortex tilting and stretching (which cannot occur in a 2D flow field), the flow in the vortex-rich region appears to be more turbulent and small scale instabilities are generated more rapidly. However, systematic boundary layer separation is not observed in the 3D simulation, and the largest bottom shear stress is associated with the lobe-cleft instability instead of the de-shoaling process.

The Rough bottom topography case has the same density profile as the Medium pycnocline case. While with a smooth bottom topography the shear stress at the edge of the core is not sufficiently strong to produce any instability, with a relatively rough bottom topography evidence of billow formation is found. This suggests that perturbation of the BBL can lead to enhanced shear stress in the leading edge of the core where the billow formation originates. On the other hand, during the wave passage boundary layer separa- 
tion is found inside each of the 'valleys' between bottom corrugations. However, because the amplitudes of these corrugations are very small, the flow is not energetic enough to self advect away from the boundary layer. As suggested in Carr et al. (2010), the energy carried by the flow inside these corrugations is determined by the size and shape of these corrugations, as well as the stratification of the fluid. To further understand the influence a rough bottom topography on the behaviour of the shoaling wave, experiments with various types of the bottom corrugations will need to be carried out.

Our numerical experiments showed that all of the wave-induced instabilities can lead to enhanced turbulence in the water column and increased shear stress on the bottom boundary. This suggests that in a near-coastal region, the shoaling and de-shoaling cycles of ISWs of elevation are likely to provide a systematic mechanism for material mixing and sediment resuspension. As physical phenomena relating to internal waves are rich while many questions remain unanswered in the present work, further investigation on the internal wave dynamics is needed to better understand this subject. One of our long term research goals is to assess the manner in which the present results scale up. Field scale fluid flows are characterized by a much higher Reynolds number than laboratory scale flows. Consequently, the flow tends to be more turbulent and the boundary layer is thinner. Hence, any instabilities generated during the wave passage are expected to result in even stronger disturbance to the overall flow field. For example, the boundary layer separation found in the Rough bottom topography case may lead to a global instability in large scale simulations. Another possible future research direction would be to examine the influence of instabilities generated by the leading wave on the entire wave train, because in nature ISWs rarely exist as single waves. Variation of the bottom topography could also be considered.

An attempt has been made to scale up the rough bottom topography simulation. This case, labelled as Scale-up, considers a 2D domain with a length of $L_{x}=70 \mathrm{~m}$ and a depth of $L_{z}=2 \mathrm{~m}$. The shelf on the bottom boundary is also scaled up proportionally and has a crest of height $\Delta H=0.25 \mathrm{~m}$ located at $x_{c}=60 \mathrm{~m}$. The functional form of the bottom topography is

$$
H(x)=-L_{z}+\Delta H e^{-h(x)}[1+\epsilon(x)],
$$

where

$$
h(x)= \begin{cases}{\left[0.05\left(x-x_{c}\right)\right]^{2},} & \text { if } x \leq x_{c} \\ {\left[0.05\left(x-x_{c}\right)\right]^{2}+0.5\left[0.2\left(x-x_{c}\right)\right]^{8},} & \text { if } x>x_{c}\end{cases}
$$

and

$$
\epsilon(x)=0.1 \sin (20 \pi x) .
$$



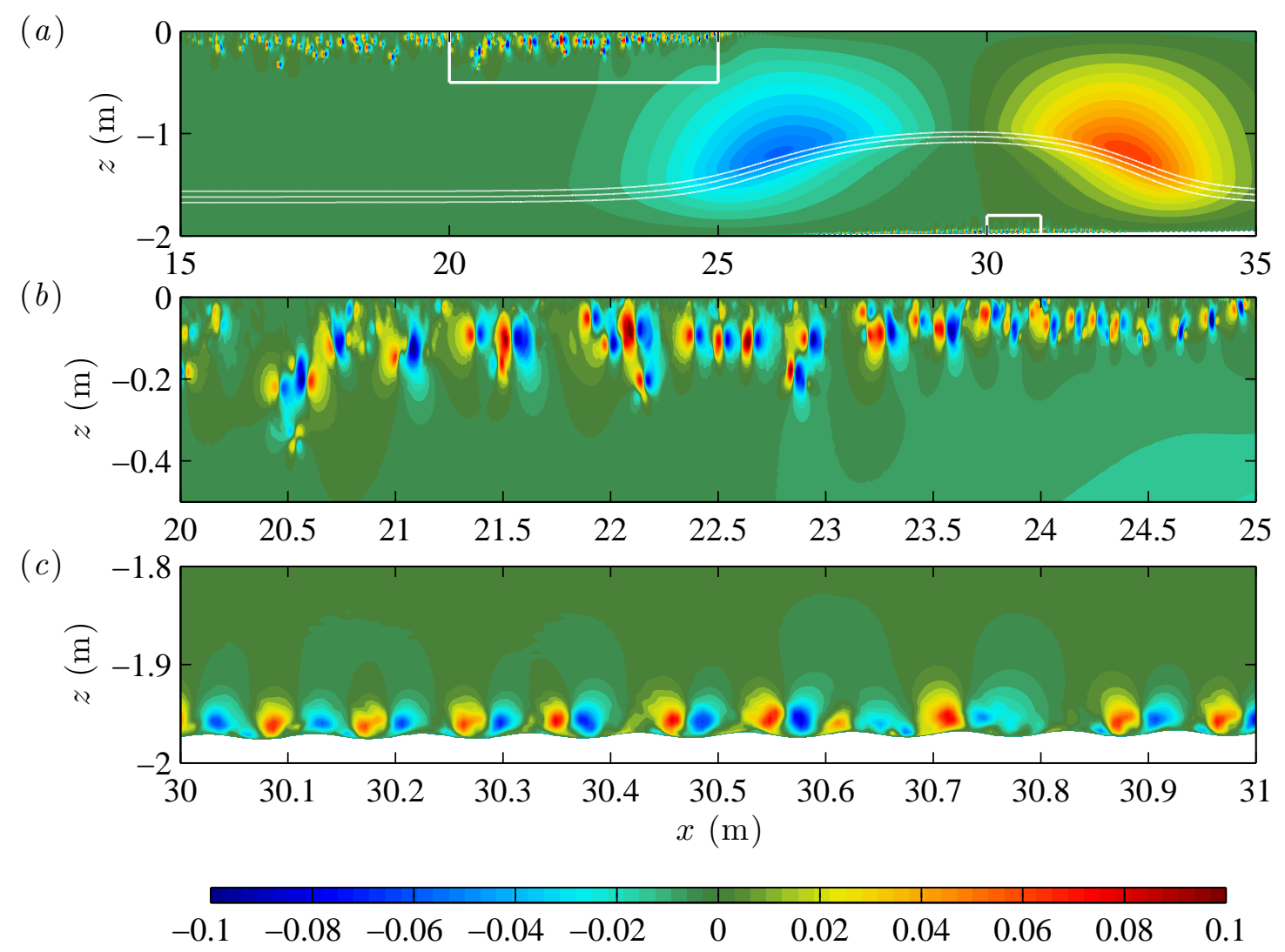

Figure 5.1: Vertical velocity plots for the Scale-up case at $t=40 \mathrm{~s}$. Panel $(a)$ : overall flow structure. Panel $(b)$ : surface boundary layer behind the main wave. Panel $c$ ): bottom boundary layer underneath the wave.

The grid size is also increased to $N_{x} \times N_{z}=12288 \times 384$ in order to achieve a similar grid spacing as the laboratory-scale simulation, such that the flow inside the BBL and the corrugations can be well resolved. The background density profile is given by

$$
\bar{\rho}(z)=1-0.5 \Delta \rho \tanh \left(\frac{z-z_{0}}{0.5 d}\right),
$$

where $\Delta \rho=0.02, z_{0}=-1.6 \mathrm{~m}$ and $d=0.2 \mathrm{~m}$. The viscosity and the diffusivity are fixed at $\nu=10^{-6} \mathrm{~m}^{2} / \mathrm{s}$ and $\kappa=5 \times 10^{-7} \mathrm{~m}^{2} / \mathrm{s}$, respectively, and the resulting Schmidt number is $S c=2$. Based on the channel half-depth and the initial wave propagation speed (which is $c=29.6 \mathrm{~cm} / \mathrm{s}$ ), the estimated Reynolds number is $R e=3 \times 10^{5}$, which is approximately 30 times the Reynolds number of the laboratory-scale simulation. 
The vertical velocity field of the Scale-up case at $t=40 \mathrm{~s}$ is shown in figure 5.1. Note that due to increased length scale the wave is still far away from the top of the bottom shelf at this time. The figure shows that the most significant differences from the laboratory-scale simulations are the vortices generated near the surface behind the main wave. This phenomenon is very similar to the vortex shedding discussed in Aghsaee et al. (2012), and is due to the no-slip and no-flux conditions imposed at the top boundary. A free-slip surface boundary condition will remove the near-surface instability, and altering the numerical model to allow a no-slip bottom but a free-slip top could be another future research direction as well. Along the bottom boundary, vortex generation due to the rough bottom boundary is also found, as shown in panel $(c)$, although in the early stages of the shoaling process these vortices are very small. Further simulation results are needed in order to determine whether these instabilities can lead to a significant difference in the wave's shoaling process. 


\section{References}

P. Aghsaee, L. Boegman, P. J. Diamessis, and K. G. Lamb. Boundary-layer-separationdriven vortex shedding beneath internal solitary waves of depression. J. Fluid Mech., 690:321-344, 2012.

L. Boegman, G. N. Ivey, and J. Imberger. The degeneration of internal waves in lakes with sloping topography. Limnol. Oceanogr., 50(5):1620-1637, 2005.

D. Bogucki, T. Dickey, and L. G. Redekopp. Sediment resuspension and mixing by resonantly generated internal solitary waves. J. Phys. Oceanogr., 27:1181-1196, 1997.

D. Bourgault, M. Morsilli, C. Richards, U. Neumeier, and D. E. Kelley. Sediment resuspension and nepheloid layers induced by long internal solitary waves shoaling orthogonally on uniform slopes. Cont. Shelf Res., 72:21-33, 2014.

M. Carr, M. Stastna, and P. A. Davies. Internal solitary wave-induced flow over a corrugated bed. Ocean Dynam., 60:1007-1025, 2010.

M. Carr, S. E. King, and D. G. Dritschel. Instability in internal solitary waves with trapped cores. Phys. Fluids, 24:016601, 2012.

A. Defina, S. Lanzoni, and F. M. Susin. Stability of a stratified viscous shear flow in a tilted tube. Phys. Fluids, 11(2):344-355, 1999.

O. G. Derzho and R. Grimshaw. Solitary waves with a vortex core in a shallow layer of stratified fluid. Phys. Fluids, 9(11):3378-3385, 1997.

P. J. Diamessis and L. G. Redekopp. Numerical investigation of solitary internal waveinduced global instability in shallow water benthic boundary layers. J. Phys. Oceanogr., 36:784-812, 2005. 
P. G. Drazin and L. N. Howard. Hydrodynamics stability of parallel flow of inviscid fluids. Adv. Appl. Mech, 9(1), 1966.

M. Dubreil-Jacotin. Sur les ondes de type permanent dans les liquides heterogenes. Atti Accad. Naz. Lincei Rend, 6:814-819, 1932.

M. Dunphy, C. Subich, and M. Stastna. Spectral methods for internal waves: indistinguishable density profiles and double-humped solitary waves. Nonlin. Processes Geophys., 18: 351-358, 2011.

S. Harnanan, N. Soontiens, and M. Stastna. Internal wave boundary layer interaction: A novel instability over broad topography. Phys. Fluids, 27:016605, 2015.

C. Hartel, F. Carlsson, and M. Thunblom. Analysis and direct numerical simulation of the flow at a gravity-current head. part 2. the lobe-and-cleft instability. J. Fluid Mech., 418: 213-229, 2000a.

C. Hartel, E. Meiburg, and F. Necker. Analysis and direct numerical simulation of the flow at a gravity-current head. part 1. flow topology and front speed for slip and no-slip boundaries. J. Fluid Mech., 418:189-212, 2000b.

K. R. Helfrich and B. L. White. A model for large-amplitude internal solitary waves with trapped cores. Nonlin. Processes Geophys., 17:303-318, 2010.

L. N. Howard. Note on a paper of john w. miles. J. Fluid Mech., 13:158-160, 1961.

J. M. Klymak and J. N. Moum. Internal solitary waves of elevation advancing on a shoaling shelf. Geophys. Res. Lett., 30(20.2045), 2003.

D. J. Korteweg and G. de Vries. On the change of form of long waves advancing in a rectangular canal, and on a new type of stationary waves. Philos. Mag., 39:422-443, 1895.

P. K. Kundu, I. M. Cohen, and D. R. Dowling. Fluid Mechanics. Academic Press, Waltham, Massachusetts, 2012.

K. G. Lamb. Theoretical descriptions of shallow-water solitary internal waves: comparisons with fully nonlinear waves. In T. F. Duda and D. M. Farmer, editors, The 1998 WHOI/IOSA/ONR Internal Solitary Wave Workshop: Contributed Papers, Woods Hole Oceanographic Institutions Tech. Rep. WHOI-99-07, 1999. 
K. G. Lamb. A numerical investigation of solitary internal waves with trapped cores formed via shoaling. J. Fluid Mech., 451:109-144, 2002.

K. G. Lamb. Shoaling solitary internal waves: on a criterion for the formation of waves with trapped cores. J. Fluid Mech., 478:81-100, 2003.

K. G. Lamb and L. Yan. The evolution of internal waves undular bores: comparisons of a fully nonlinear numerical model with weakly nonlinear theory. J. Phys. Oceanogr., 26: 2712-2734, 1996.

C. Loken, D. Gruner, L. Groer, R. Peltier, N. Bunn, M. Craig, T. Henriques, J. Dempsey, C. Yu, J. Chen, L. J. Dursi, J. Chong, S. Northrup, J. Pinto, N. Knecht, and R. Van Zon. Scinet: Lessons learned from building a power-efficient top-20 system and data centre. J. Phys.: Conf. Ser., 256(012026), 2010.

R. Long. Some aspects of the flow of stratified fluid: I. a theoretical investigation. Tellus, $5: 42-58,1953$.

V. Maderich, T. Talipova, R. Grimshaw, K. Terletska, I. Brovchenko, E. Pelinovsky, and B. H. Choi. Interaction of a large amplitude interfacial solitary wave of depression with a bottom step. Phys. Fluids, 22:076602, 2010.

J. W. Miles. On the stability of heterogeneous shear flows. J. Fluid Mech., 10:496-508, 1961.

C. Richards, D. Bourgault, P. S. Galbraith, A. Hay, and D. E. Kelley. Measurements of shoaling internal waves and turbulence in an estuary. J. Geophys. Res. Oceans, 118: 1-14, 2013.

C. Scalo, U. Piomelli, and L. Boegman. High-schmidt-number mass transport mechanisms from a turbulent flow to absorbing sediments. Phys. Fluids, 24:085103, 2012.

A. Scotti and J. Pineda. Observation of very large and steep internal waves of elevation near the massachusetts coast. Geophys. Res. Lett., 31(L22307), 2004.

J. E. Simpson. Effects of the lower boundary on the head of a gravity current. J. Fluid Mech., 53:759-768, 1972.

J. E. Simpson and R. E. Britter. The dynamics of the head of a gravity current advancing over a horizontal surface. J. Fluid Mech., 94:477-495, 1979. 
N. Soontiens. Stratified Flow Over Topography: Steady Nonlinear Waves, Boundary Layer Instability, and Crater Topography. PhD thesis, University of Waterloo, 2013.

M. Stastna. Large Fully Nonlinear Solitary and Solitary-like Internal Waves in the Ocean. PhD thesis, University of Waterloo, 2001.

M. Stastna and K. G. Lamb. Large fully nonlinear internal solitary waves: The effect of background current. Phys. Fluids, 14(9):2987-2999, 2002.

M. Stastna and K. G. Lamb. Sediment resuspension mechanisms associated with internal waves in coastal waters. J. Geophys. Res., 113:C10016, 2008.

C. J. Subich. Simulation of the Navier-Stokes Equations in Three Dimensions with a Spectral Collocation Method. PhD thesis, University of Waterloo, 2011.

C. J. Subich, K. G. Lamb, and M. Stastna. Simulation of the navier-stokes equations in the three dimensions with a spectral collocation method. Int. J. Numer. Mech. Fluids, 73:103-129, 2013.

T. Talipova, K. Terletska, V. Maderich, I. Brovchenko, K. T. Jung, E. Pelinovsky, , and R. Grimshaw. Internal solitary wave transformation over a bottom step: Loss of energy. Phys. Fluids, 25:032110, 2013.

S. A. Thorpe. Experiments on the instability of stratified shear flows: Miscible fluids. $J$. Fluid Mech., 46:299-319, 1971.

L. N. Trefethen. Spectral Methods in Matlab. Society for Industrial and Applied Mathematics, Philadelphia, Pennsylvania, 2000.

S. K. Venayagamoorthy and O. B. Fringer. On the formation and propagation of nonlinear internal boluses across a shelf break. J. Fluid Mech., 577:137-159, 2007. 